\title{
Elastic guided wave dispersion in layered piezoelectric plates: Application to ultrasound transducers and acoustic sensors
}

\author{
Daniel H. Cortes Correales \\ West Virginia University
}

Follow this and additional works at: https://researchrepository.wvu.edu/etd

\section{Recommended Citation}

Cortes Correales, Daniel H., "Elastic guided wave dispersion in layered piezoelectric plates: Application to ultrasound transducers and acoustic sensors" (2009). Graduate Theses, Dissertations, and Problem Reports. 3487.

https://researchrepository.wvu.edu/etd/3487

This Dissertation is protected by copyright and/or related rights. It has been brought to you by the The Research Repository @ WVU with permission from the rights-holder(s). You are free to use this Dissertation in any way that is permitted by the copyright and related rights legislation that applies to your use. For other uses you must obtain permission from the rights-holder(s) directly, unless additional rights are indicated by a Creative Commons license in the record and/ or on the work itself. This Dissertation has been accepted for inclusion in WVU Graduate Theses, Dissertations, and Problem Reports collection by an authorized administrator of The Research Repository @ WVU.

For more information, please contact researchrepository@mail.wvu.edu. 


\title{
Elastic Guided Wave Dispersion in Layered Piezoelectric Plates: Application to Ultrasound Transducers and Acoustic Sensors
}

\author{
Daniel H. Cortes Correales
}

\begin{abstract}
Dissertation
Submitted to the College of Engineering and Mineral Resources at West Virginia University in partial fulfillment of the requirements for the degree of
\end{abstract}

\section{Doctor of Philosophy \\ In \\ Mechanical Engineering}

\begin{abstract}
Osama Mukdadi, PhD., Committee Chair Ever Barbero, PhD., Committee Member Tim McGraw, PhD., Committee Member Victor Mucino, PhD., Committee Member Nithi Sivaneri, PhD., Committee Member
\end{abstract}

Department of Mechanical and Aerospace Engineering

Morgantown, West Virginia

2009

Keywords: Guided Waves, Ultrasound Transducers, Harmonic Imaging, Acoustic Sensors 


\section{Abstract \\ Elastic Guided Wave Dispersion in Layered Piezoelectric Plates: Application to Ultrasound Transducers and Acoustic Sensors}

\section{Daniel H. Cortes}

Elastic wave propagation in layered media has been of interest in many disciplines including non-destructive material characterization, acoustic sensors and medical ultrasound imaging. A great variety of devices are based on the propagation of electromechanical waves in piezoelectric plates. Despite the diversity of applications, the principles and characteristics of wave propagation are the same regardless the design or configuration of the device. The aim of this study is to develop numerical and analytical tools to model and design devices based on the wave propagation in piezoelectric layered media; particularly, ultrasound transducers for medical imaging and acoustic sensors for biological applications.

Single-element ultrasound transducers can be modeled as infinite layered piezoelectric plates. A Semi-Analytical Finite Element (SAFE) method has been implemented and used to theoretically predict the resonant frequencies and the dispersion behavior of these plates. The analysis of piezoelectric layered plates showed that the resonant frequencies at the ZGV points of the Lamb wave modes are more significant than those at the cut-off frequencies. On the other hand, 1D array ultrasound transducers are modeled as periodic piezoelectric plates with finite cross-section using a modified SAFE method. Dispersion curves, group velocity spectra and mode shapes are obtained using this method. Geometric parameters of the piezoelectric element, such as, aspect ratio and subdicing width and depth of the piezoelectric element had an important effect on the dispersion behavior. In general, the lower aspect ratios, as well as lower subdicing depth, tend to increase the cut-off frequency and the resonance of all modes.

An analytical model is presented to study the dynamic behavior of single-element ultrasound transducers and acoustic sensors. This model is based on leaky Rayleigh and Lamb wave analysis, which has been widely used for NDT applications. A procedure to calculate the resonance frequencies for ultrasound transducers based on the dispersion curves has been derived and experimentally validated. This analytical solution has also been used to calculate and optimize the sensitivity and coupling coefficient of Rayleigh and Lamb wave sensors.

The procedures and results obtained in this study can be used to analyze and design devices based on the wave propagation on multilayered piezoelectric plates, i.e. ultrasound transducer and sensors. It is believed that an accurate characterization of the wave propagation and dynamic properties, as well as an optimized configuration of the device can substantially improve its performance. These developments can be applied on ultrasound biomicroscopy, medical imaging, NDT techniques, MEM devices and biosensors. 
To my wife Nohemy, my parents Martha and Baltazar and my sister Marcela 


\section{Acknowledgements}

This study was carried out during the years 2006-2009 at the Mechanical and Aerospace Engineering Department of West Virginia University. This study was made possible by contribution of many important people.

Most of all, I am deeply thankful to my loving wife Nohemy, for her unconditional support and understanding during the past twelve years.

I am forever grateful to my parents Martha and Baltazar for all the valuable lessons they have taught throughout my entire life, and for the effort undertaken while investing in my education. Also, I am grateful to my sister Marcela for her company and support.

I am grateful to Dr. Barbero for his advice, especially during the application process to WVU.

I am also sincerely thankful to Dr. Mukdadi for his advice and the countless hours dedicated to this project.

I want also to thank the committee members for their time and advice in this study.

I am also grateful to my colleagues Ahmed and Vincent for their help on the experimental side of this study.

Finally but not less important, I am grateful to my friends in Colombia and Morgantown for ameliorating the stress inherent to Ph.D. studies. 


\section{Table of Contents}

1 General Introduction -- 1

1.1 Scope of this study---

1.2 Objectives----------------------------------------------------

1.3 Outline of this thesis--

2 Literature Review --

2.1 Harmonic Imaging --

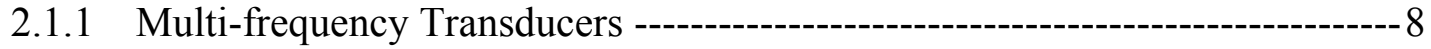

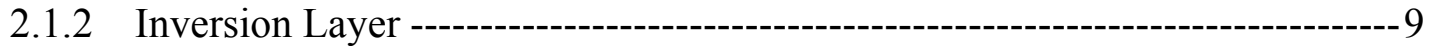

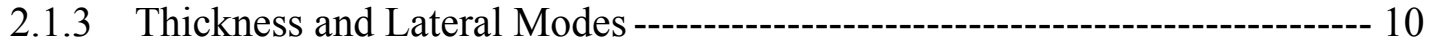

2.2 Acoustic Sensors - 10

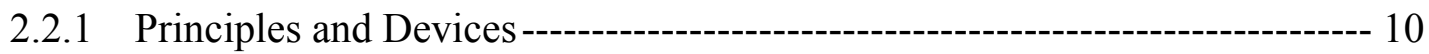

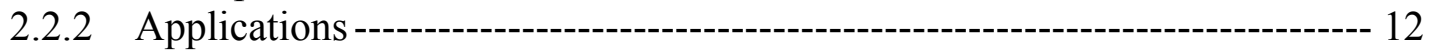

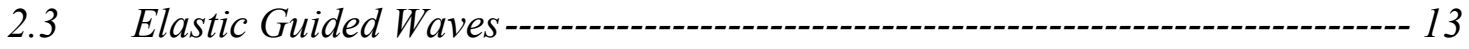

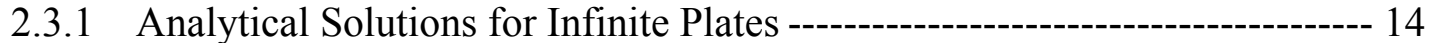

2.3.2 Finite Element Modeling ---o- 15

3 Infinite Plate Model for Single Element Transducers --- 16

3.1 Finite Element Formulation------------------------------------- 17

3.2 Electro-Elastodynamic Green's Functions-------- 19

3.3 Frequency Response Due to Impulse Loading--------------------------- 20

$3.4 \quad$ Numerical Results and Discussion ---------------------------------------- 21

3.5 Concluding Remarks --- 27

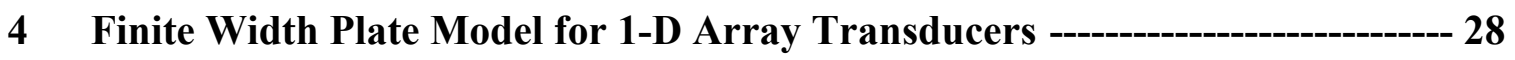

4.1 Finite Element Formulation------ 29

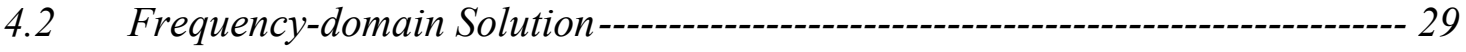

4.3 Boundary Conditions----------------------------------------------- 30

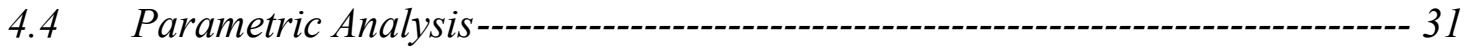

4.5 Results and Discussion ----------- 32

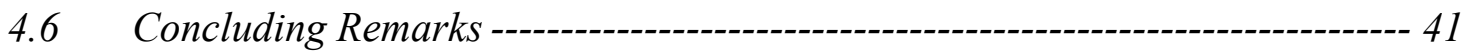

5 Analytical Modeling of Single-Element Ultrasound Transducer using Leaky Lamb Waves ----- 43

5.1 Introduction -- 44

5.2 Analytical Model-- 44

5.2.1 Model Description---_- 44 


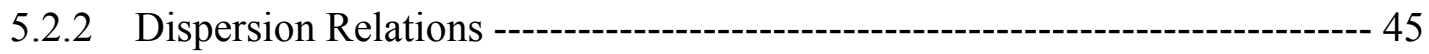

5.2.3 Boundary Conditions--------------------------------------------- 46

5.2.4 Frequency Response to an External Force --------o- 48

5.3 Experimental Validation

5.4 Results and Discussion -- 49

5.4.1 Validation----o--- 50

5.4.2 Effect of the backing layer on the dispersion curves and resonance frequencies -------------------------------------------------------- 52

5.4.3 Effect of fluid loading on the dispersion curves and resonance frequencies 56

5.4.4 Effect of matching layers on the dispersion curves and resonance frequencies 57

5.5 Conclusions --- 58

6 Modeling and Optimization of Acoustic Sensors ---_e 60

6.1 Introduction --- 61

6.2 Model Description------------ 63

6.3 Coupling Coefficient ----- 63

6.4 Wavelet Analysis------------------------------------------------------------ 65

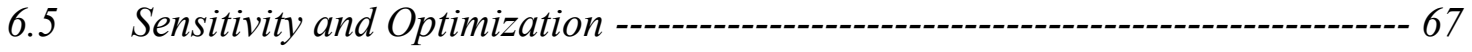

6.6 Conclusions -- 71

7 Conclusions and Future Work -

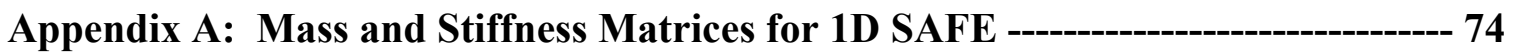

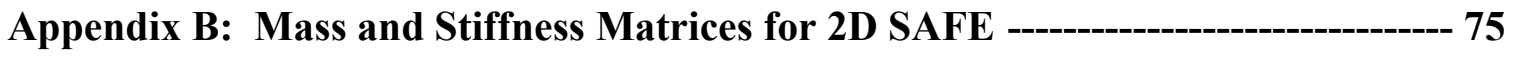

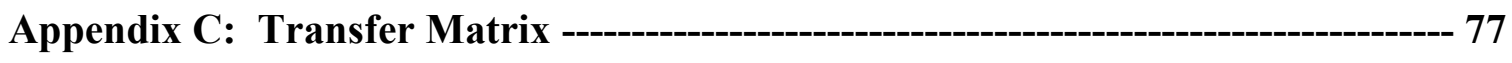

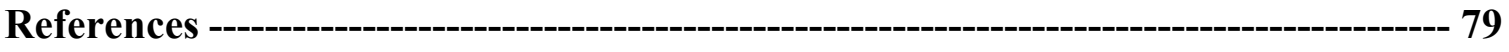




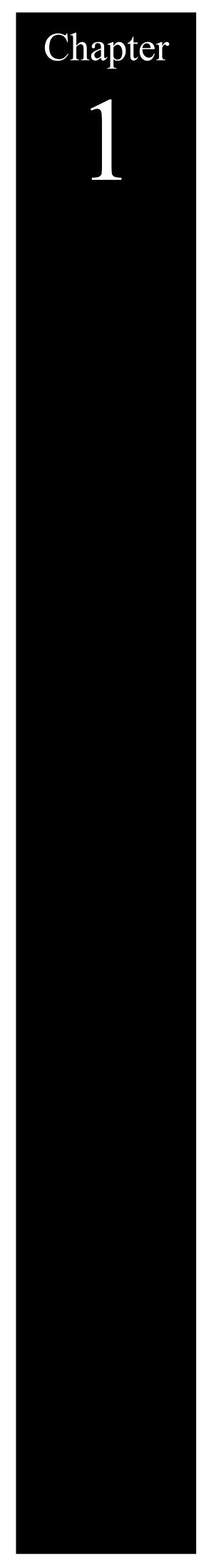

General Introduction 


\subsection{Scope of this study}

Vibration and wave propagation in layered media has been of interest in the fields of geophysics, acoustics, MEMs, etc. A great variety of devices are based on the propagation of electromechanical waves in plates. Despite the diversity of applications, the principles and characteristics of wave propagation are the same regardless the design or configuration of the device. Two main applications have been considered for this study: ultrasound transducer for medical applications and acoustic sensors.

Ultrasound medical imaging is a safe, low-cost, versatile technique routinely used in medical evaluation and diagnosis. The details shown in the image are extremely important for physicians and clinicians. Therefore, one important research task in ultrasound imaging is the improvement of image contrast and resolution. Several techniques are currently studied to enhance the contrast of ultrasound images. Tissue Harmonic Imaging (THI) is a technique in which the signal is excited at a fundamental frequency $f_{0}$ and the image is constructed using received signals at harmonic frequencies $2 f_{0}, 3 f_{0}$ and so forth. These higher harmonic frequency signals are generated due to the cumulative nonlinearity of the tissue. The tissue nonlinearity becomes a significant factor as the wave propagates relatively large distance in the tissue. Another interesting nonlinear imaging method is the use of ultrasound contrast agents (microbubbles) for contrast-enhanced ultrasound imaging. This technique employs contrast agents or microbubbles, which generate harmonics due to the local nonlinearity of contrast agents surrounded by connective tissues. THI requires sending and receiving ultrasound signal at several frequencies, hence, ultrasound transducers must have high efficiency at these frequencies. Design of such transducers is a challenging task that requires a careful selection of materials, dimensions and configuration.

On the other hand, ultrasonic biosensors are devices used to detect changes in the environment or the presence of viruses or contaminants in the surroundings. The principle behind all these sensors is that a change in the environment will produce a measurable variation of one or several parameters of the wave propagation. Some sensors have a special layer, which absorbs or attract substances or viruses causing a change in the mechanical properties of the layer and consequently a change on the characteristics of the wave propagation (Gronewold, 2007). Other sensors do not change their properties, but the interaction between the sensor and the surrounding fluid allow them to 'sense' a change in the properties of the surrounding fluid, such as density, viscosity, etc. These sensors are based on the propagation of 'leaky' surface acoustic waves (Lindner, 2008). Leaky waves are characterized by an exchange of energy between the solid and fluid. This effect produces a reduction or attenuation of the amplitude of the wave along the direction of propagation. The severity of this attenuation is function of the frequency and the properties of the fluid (Nayfeh, 1995). For a given frequency, a change in the attenuation is an indication of a change of the fluid properties. In addition to attenuation, a reduction in the wave velocity is also observed. Therefore, wave velocity can be also used as a probe to measure changes in the fluid.

Although ultrasound transducers and ultrasonic biosensor are devices with different functions, they have several common characteristics. Usually they make use of 
the piezoelectric effect to convert electric signals into vibrations and vice versa; therefore, they are composed of a combination of elastic and piezoelectric layers. The principle of both devices is based on the generation and propagation of ultrasound waves; consequently, in order to model and design them is important to accurately analyze the behavior of electromechanical waves as well as determine how these waves are affected by the material properties and dimension of each of their components. The aim of this study is to develop numerical and analytical tools to model and design devices based on the wave propagation in piezoelectric layered media; particularly, ultrasound transducers for medical imaging and biosensors. It is believed that an accurate characterization of the wave propagation and dynamic properties, as well as an optimized configuration of the device can substantially improve its performance. These developments can be applied on ultrasound biomicroscopy, medical imaging, NDT techniques, MEM devices and biosensors.

\subsection{Objectives}

The general purpose of the project is to develop and apply numerical and analytical tools to optimize the design of ultrasound transducers and biosensors. Our specific aims are

1) Implement the semi-analytical finite element method to analyze infinite multilayered plates.

Rationale: A first approach to model ultrasound transducer and sensors is a multilayered plate composed of one of several piezoelectric layers and other elastic (passive layers). This model can be used to numerically study important features in the wave propagation in plates such as wave velocities, dispersion curves and resonance frequencies.

2) Implement and Develop a $2 \mathrm{D}$ semi-analytical finite element method with periodic boundary conditions to analyze array transducers.

Rationale: Array transducers are composed of many piezoelectric elements that can individually excite and record mechanical waves. A 2D SAFE method with periodic boundary conditions can be used to analyze the complete array considering just one element of the array. In addition, complicated geometries of piezoelectric element can be easily studied.

3) Develop analytical solutions to analyze guided wave propagation of infinite plates with different boundary conditions to account for the effect of surrounding media, like fluid, tissue, substrates, etc.

Rationale: Ultrasound transducers and acoustic sensors can be represented by a multilayered plate surrounded by a half-space solid (representing the backing layer or substrate) and half-space fluid (tissue or environment). Therefore, a model that includes the effects of the surrounding media is needed to accurately characterize these devices. It is expected that the dynamic behavior described by this model closely matches experimental measurements.

4) Model and Optimize the sensitivity of ultrasonic biosensors.

Rationale: The sensitivity of ultrasonic biosensor depends on the change of the wave propagation characteristics due to a change in the surrounding environment. The numerical and analytical solutions proposed in this study can be used to quantitatively determine and maximize the sensitivity optimizing geometrical parameters of the device. 
5) Evaluate and validate the proposed modeling techniques taking measurements of resonance frequencies and velocities on transducers and sensors.

Rationale: Prototype devices will be fabricated and experimental measurements will be performed to validate the results obtained from the numerical and analytical models. Comparison of resonance frequencies and wave velocities, which are the most important parameters for these devices, will determine the accuracy of the proposed models.

\subsection{Outline of this thesis}

Chapter 2 presents a compilation of the relevant previous studies which were used as the basis of this study. This chapter is divided in three sections. One section is devoted to the previous studies on the wave propagation and dispersion analysis of guided waves; a second section about ultrasound harmonic imaging and transducer design is presented; and finally, a review of the state of the art for acoustic sensors is presented.

Chapters 3 and 4 deal with the analysis of ultrasound transducers using a SemiAnalytical Finite Element (SAFE) method. Chapter 3 presents the analysis of single element transducers using 1D SAFE method to obtain the dispersion curves and their relation with resonance frequencies. Chapter 4 presents a similar analysis for array transducers using a $2 \mathrm{D}$ version of SAFE technique which includes periodic boundary conditions.

Chapter 5 presents the analytical solution for the wave propagation and resonance frequencies on multilayered plates. This solution includes boundary conditions such as half-space fluid and half-space solids, which can not be considered using 1D SAFE. Additionally, a procedure to obtain the resonance frequencies was developed and validated. This solution was used to analyze the mechanical behavior of single-element ultrasound transducer.

Based on the results of chapter 5 , it was found that this solution can also be used to model acoustic sensors. Chapter 6 presents the modeling of ultrasonic sensors based on Rayleigh and Lamb waves. Important characteristics of the sensor such as coupling factor and sensitivity were analytically obtained. Finally, an optimization procedure to obtain important geometrical parameter is proposed.

Chapter 7 presents a summary of the important conclusions and future work. 


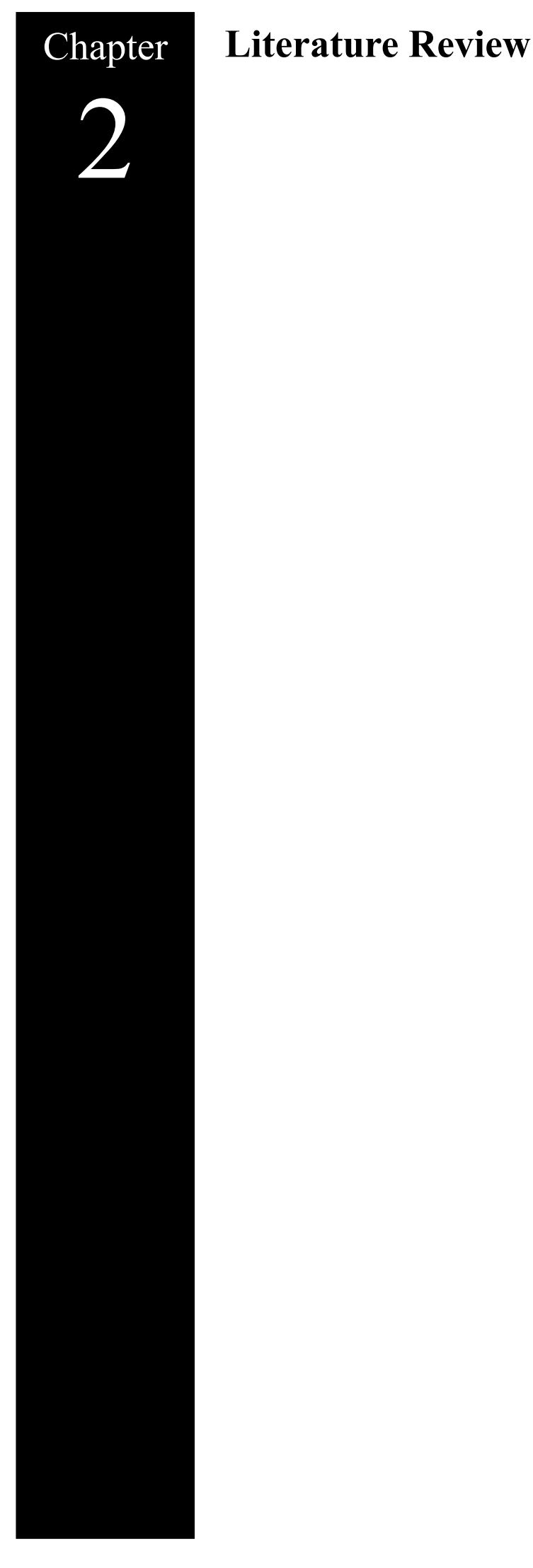




\subsection{Harmonic Imaging}

Ultrasound harmonic imaging began when engineers and clinicians accidentally found a second harmonic component on the received ultrasound signals. This effect was first related to problems in the instrumentation. However, the signals at this harmonic frequency had similar characteristics to those at the fundamental frequency. Therefore, it was possible to construct ultrasound images using harmonic signals. Explanation of this phenomenon pointed to the acoustical nonlinearity of tissue (Spencer et al., 1998; Kornbluth et al., 1998). The interest in harmonic images was increased when clinicians started to favor second-harmonic images due to the details and better contrast in the images (Fig. 2.1).

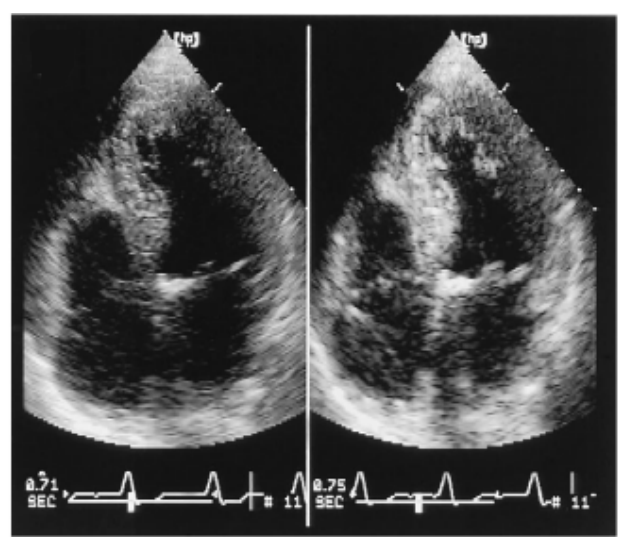

Figure 2.1. Comparison between ultrasound images of the heart ventricles constructed with fundamental frequency (left) and the second harmonic (right) (Spencer et al., 1998).

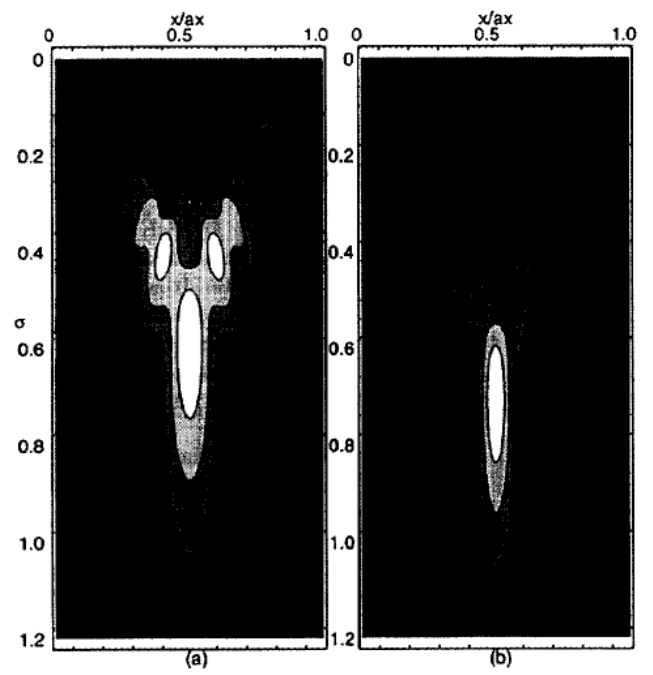

Figure 2.2. Comparison between ultrasound fields for focused ultrasound. Fundamental frequency (left) and the second harmonic (right). (Averkiou, 2000).

The increase of contrast has been explained by Spencer et al., 1998; Tranquart et al., 1999; Humphrey, 2000. The resolution along the ultrasound beam direction is related to the wavelength of the signal. At higher frequencies the wave length is smaller and better resolution is expected. The ultrasound field at the fundamental and second 
harmonic also presents differences that favor the second harmonic signal (Averkiou, 2000). For instance a comparison of the ultrasound field for focused ultrasound shows that the second harmonic field presents fewer amounts of lateral peaks (Fig. 2.2).

Second-harmonic ultrasound imaging technique has been well studied and established. However, recent studies have explored the possibility of using not only the second but also other higher harmonics (Bouakaz et al., 2002; Akiyama et al., 2005). The advantage of using higher harmonics is that even better contrast can be obtained. However, the major disadvantage is that harmonic signals are very weak and it is very difficult to acquire then with a good signal-to-noise ratio. Bouakaz et al. (2002) used a dual frequency transducer with two center frequencies one at the fundamental frequency $f_{0}$ and the second one around the fourth harmonic. The comparison between the images obtained using the second and the third through fifth harmonic shows a big improvement of the contrast (Fig. 2.3). Akiyama et al. (2005) constructed ultrasound images combining the first nine harmonic images. From the reconstructed images it is clearly seen that for every addition of a harmonic signal, the image contrast is improved (Fig. 2.4).

Conventional ultrasound transducers have a frequency bandwidth around $40 \%$. This means that efficiency of the transducer is high (or the losses are low) in a frequency range of the $40 \%$ of center frequency of the transducer. However, a higher frequency bandwidth is needed to excite a signal with a frequency $f_{0}$ and receive the harmonic component at $2 f_{0}$. Several solutions have been proposed to overcome this problem. However, the development for high efficiency transducer to detect second and higher harmonics is still the objective of many currently research projects.
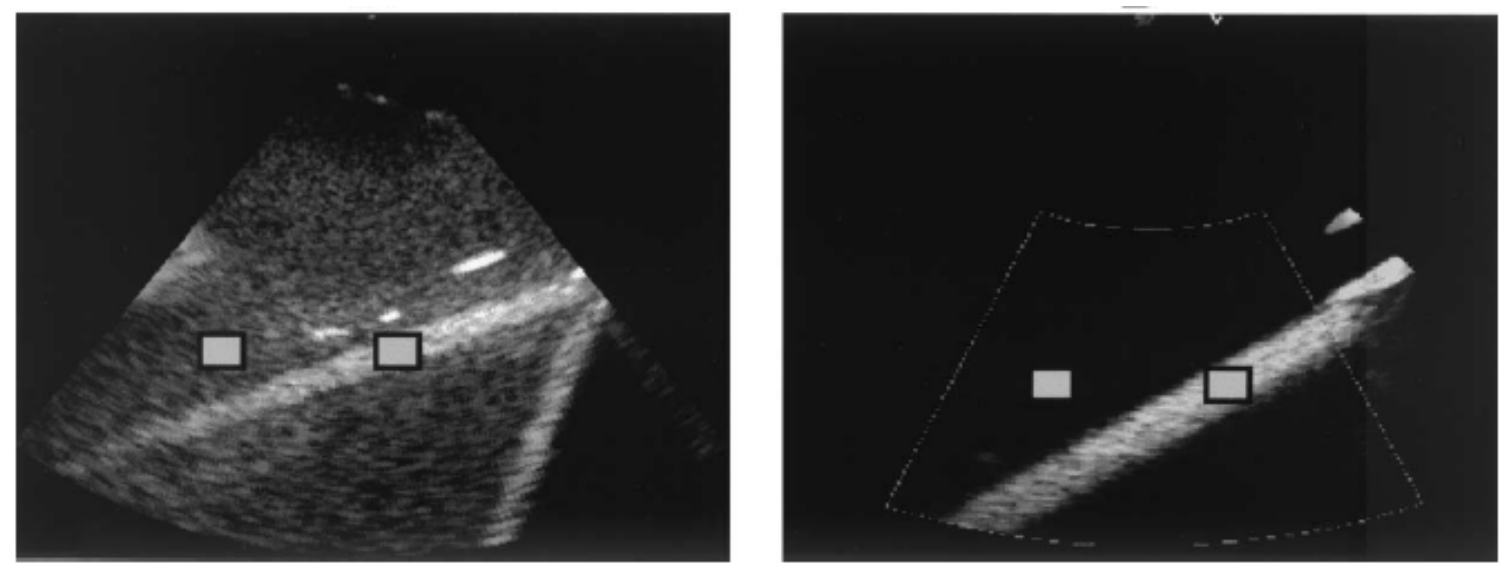

Figure 2.3. B-mode images of an ultrasound phantom constructed with the second harmonic (left) and the third through fifth harmonics (right). (Bouakaz et al., 2002). 


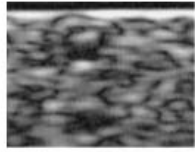

(a)

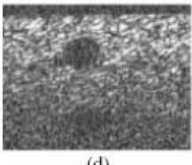

(d)

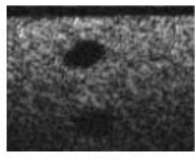

(g)

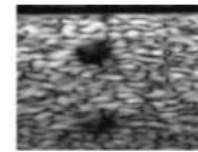

(b)

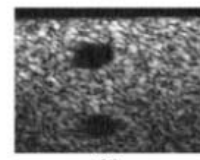

(e)

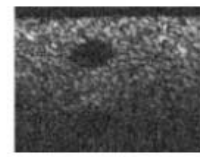

(b)

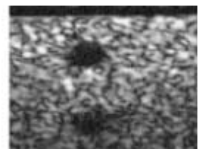

(c)

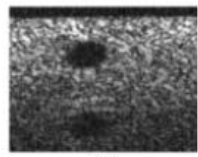

(f)

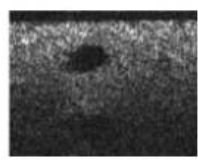

(i)

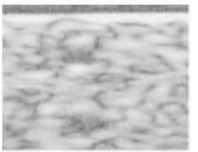

(a)

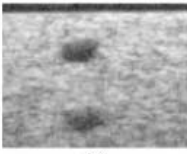

(d)

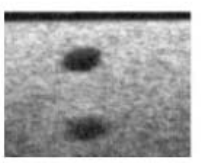

(g)

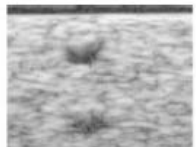

(b)

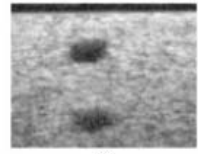

(e)

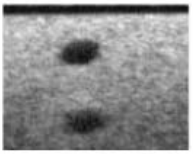

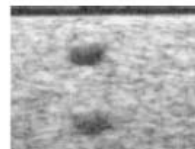

(c)

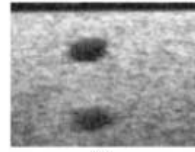

(f)

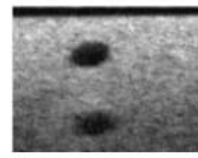

Figure 2.4. Experimental images obtained using each of the nine harmonics (left) and images combining images from the fundamental frequency through the ninth harmonic (right). (Akiyama et al., 2005).

\subsubsection{Multi-frequency Transducers}

One of the earliest attempts to increase the frequency bandwidth was the use transducers with piezoelectric elements designed to operate at several frequencies (Bouakaz et al., 2002; Akiyama et al., 2005; von Ramm and Smith, 1978). Von Ramm and Smith (1978) designed a 1D array with two rows of piezoelectric elements with center frequencies of $1.5 \mathrm{MHz}$ and $2.5 \mathrm{MHz}$, respectively. Although their aim was to increase the overall bandwidth of the transducer, this idea has been used to generate designs of multi-frequency transducer for harmonic imaging. In their study, it was proven that using wider bandwidth transducer some problems of ultrasound such as grating lobes were reduced. A recent study by Bouakaz et al. (2002) presented a design of a 1D array transducer in which the even and the odd elements of the array had different center frequencies. This configuration allowed them to obtain images up to the fifth harmonic. Later, Akiyama et al. (2005) used a conventional single element ultrasound transducer at center frequency of $2 \mathrm{MHz}$ with a bandwidth of $31 \%$ and a ring-like transducer with a $7 \mathrm{MHz}$ center frequency and a bandwidth of $100 \%$. Many other configurations have been also proposed in the literature.

Another important concept of transducer design proposed for THI is the use of multilayer transducers (Powell et al., 1998; Ramesh et al., 2006; Mills and Smith, 2002; Saitoh et al., 1995). In general, the piezoelectric elements in these transducers are composed of several layers, usually of the same material, with electrodes between them (Fig. 2.5). This configuration has been used to improve the reception, emission and bandwidth of transducers. Comparison between a multi-layer and a single layer piezoelectric element of the same thickness indicates that layered configuration produces larger displacements. This is caused by the increase in the electric field due to proximity of the electrodes. 

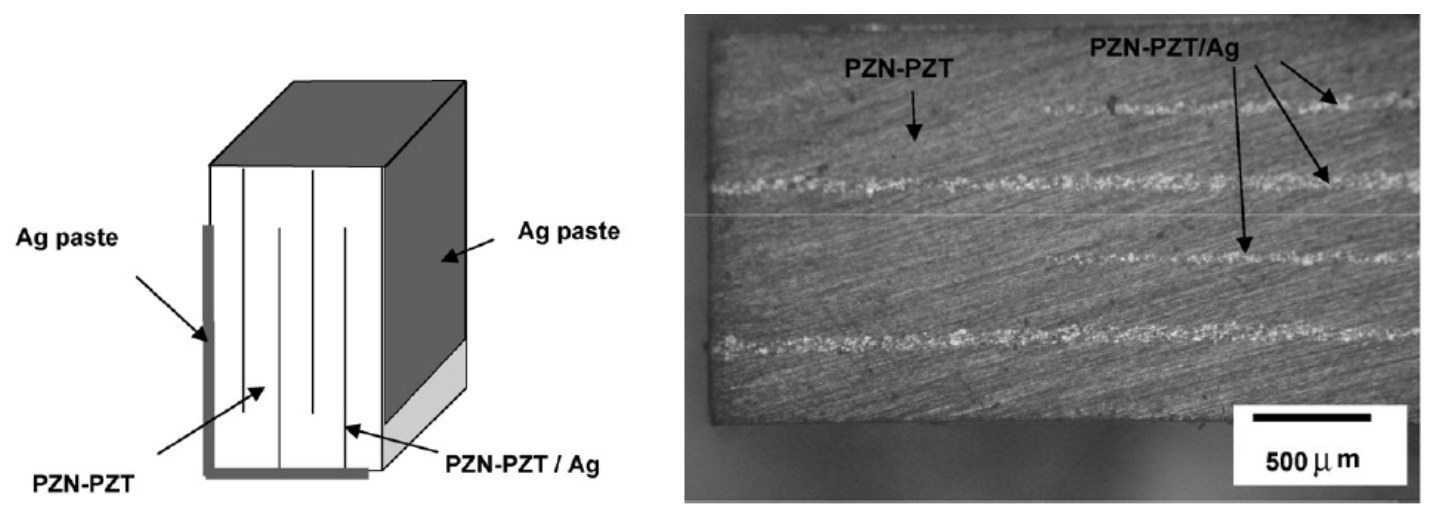

Figure 2.5. Multilayer piezoelectric transducer: a) schematic illustration, b) optical micrograph of the piezoelectric element.

\subsubsection{Inversion Layer}

Inversion Layer (IL) transducers are a special kind of multilayered transducer in which the piezoelectric properties of a portion of the thickness are inverted by mean of a heat treatment of $\mathrm{LiNbO}_{3}$ crystals (Nakamura et al., 1992, Mayazawa, 1979). Inversion of the piezoelectric properties was reported by Mayazawa (1979) as an undesirable effect in $\mathrm{LiNbO}_{3}$ crystal when a Ti film was deposited on the surface. Later Nakamura et al. (1992) achieved IL growth of even $\mathrm{LiNbO}_{3}$ plates at temperatures above $1070{ }^{\circ} \mathrm{C}$ (Fig. 2.6).

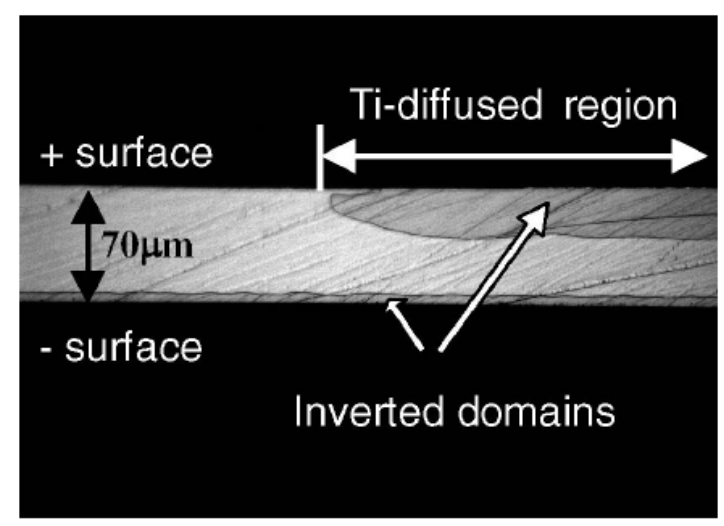

Figure 2.6. Cross section of a plate of $\mathrm{LiNbO} 3$ with inverted domains due to heat treatment. (Nakamura et al., 2006).

An important characteristic of single element IL transducers, reported in several studies, is that even-order as well as odd-order modes can be piezoelectrically excited. An early study of Saitoh et al. (1995) showed that a two-layer transducer with inverted poling directions can be used to construct a dual frequency ultrasonic probe. Analytical models of IL transducers solving the wave propagation problem (Saitoh et al., 1995) or using the Mason's equivalent circuit (Nakamura et al., 2005) have shown better efficiency at the fundamental and the second harmonic frequency. Time-domain finite-element simulations have been also used to predict the performance of IL transducers (Zhou et al., 2006). Cortes et al. (2008) studied the effect of IL on the resonance frequencies and mode shapes of multilayered piezoelectric plates using semi-analytical finite-element 
(SAFE) method. This study was also focused to the analysis of the mechanical behavior of single-element transducers.

\subsubsection{Thickness and Lateral Modes}

The aspect ratio of the cross-section of the piezoelectric elements of a 1D array transducer has been found to play an important role on their resonance frequencies (Onoe and Tiersten, 1963; Wang and Chang, 2003). For rectangular cross-section, a coupling between the thickness and lateral modes is usually observed. This behavior can be approximately predicted by mean of the coupling theory (Onoe and Tiersten, 1963; Chan and Unsworth, 1987). In this theory, the relationship between the first thickness and lateral resonance frequencies is approximated by a biquadratic equation. Wang and Chan (2003) found that an aspect ratio of 1.45 produces two resonance peaks on the frequency spectrum of the transducer. Since the coupling theory only relates the first resonance frequency in each direction, it would be desirable for broadband transducer design to analyze the effect of the aspect ratio on high-order thickness and lateral resonance frequencies.

\subsection{Acoustic Sensors}

\subsubsection{Principles and Devices}

An acoustic sensor is a device used to detect changes in its surrounding environment using electromechanical waves as a probe. The sensor consists of an elastic material used as a substrate and one or several piezoelectric layers on top. White and Voltmer (1965) demonstrated that surface waves can be generated and detected efficiently using Interdigital Transducers (IDT). An IDT consists of a series of parallel metal electrodes periodically spaced on the surface of the piezoelectric layer (Figure 2.7). When a voltage is applied to these terminals, electric fields are generated in the piezoelectric material, and these excite alternating stress patterns due to the piezoelectric effect. These induced stresses generate a mechanical wave that propagates away from the IDT. The mechanical wave is then detected by a second IDT located a given distance from the emitter.

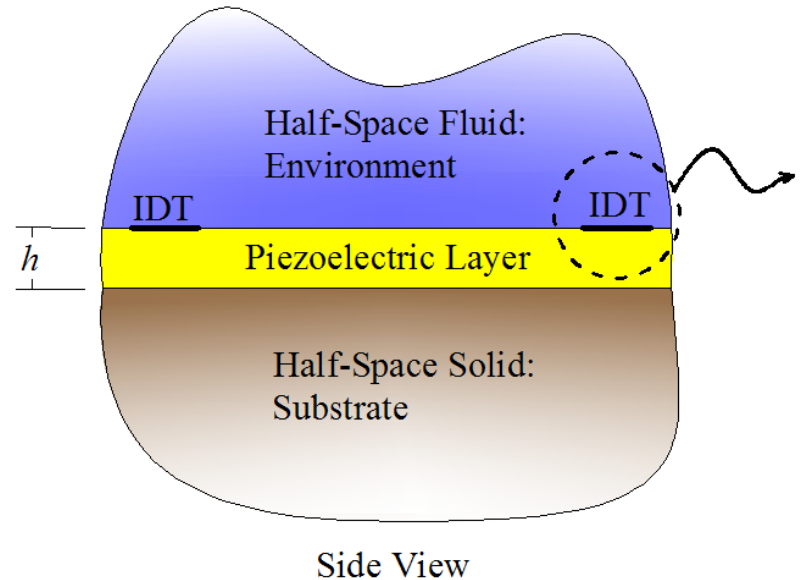

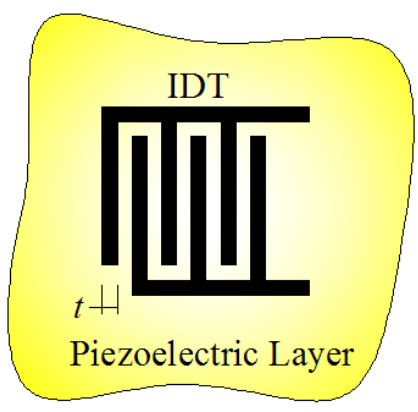

Top View

Figure 2.7. Geometry of a surface acoustic sensor. 
The coupling coefficient is an important parameter of the sensor, which quantify the effect of piezoelectricity on the wave propagation, and allows comparison between several types of configurations (Kallard, 1971). The presence of piezoelectricity increases the velocity of the wave above the value it would have if the components of the piezoelectric tensor were equal to zero. If the surface of the sensor is electrically shortened, as produced by a conducting layer placed on the surface, the piezoelectric effect would be partially 'switched off' (Kino, 1987). The change in the wave velocity due to this boundary condition is then a measurement of the piezoelectric effect. Therefore, the coupling coefficient is defined as $k^{2}=2 \Delta v / v$. It has been found (Kallard, 1971; El Hakiki et al., 2004; Benetti et al., 2005) that the coupling coefficient changes when placing the IDTs at the interface between the piezoelectric layer and the substrate or between piezoelectric layers. Figure 2.8 shows a comparison of the coupling coefficient for several configurations as a function of the thickness to wavelength ratio. It is evident that the coupling coefficient depends on the sensor configuration and the wavelength.

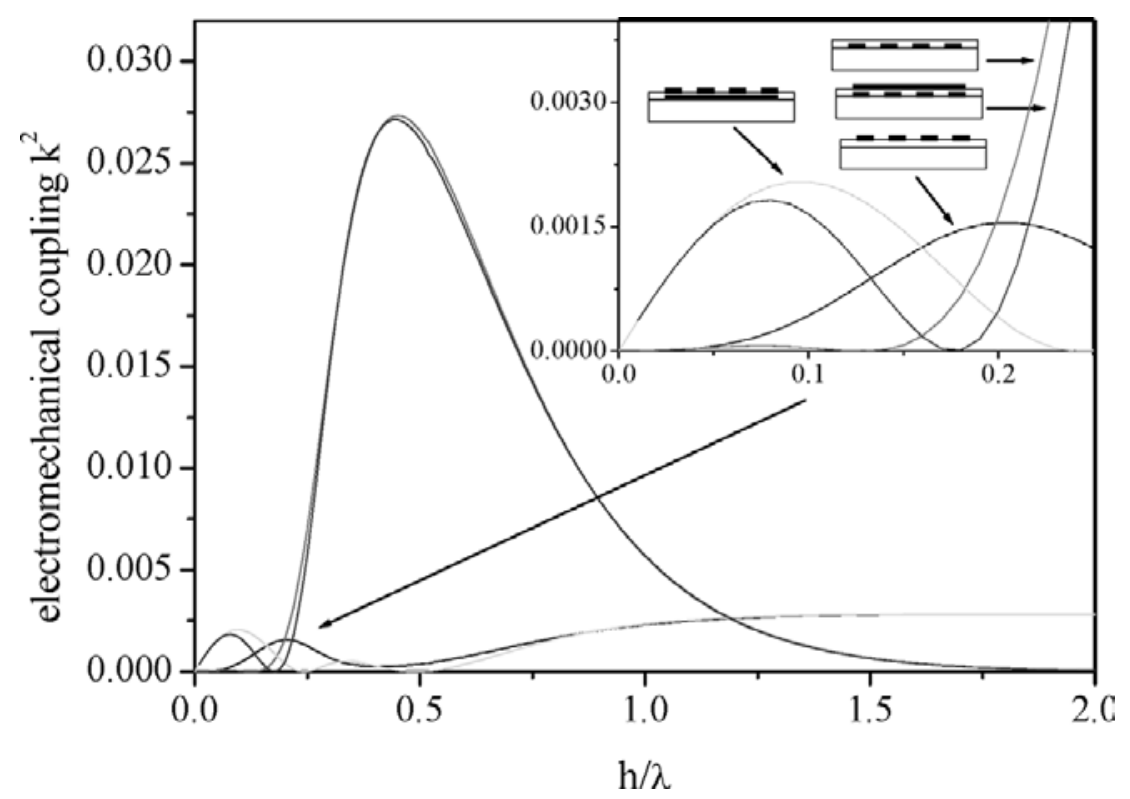

Figure 2.8. Coupling coefficient of an aluminum nitride film on a diamond substrate (Benetti et al., 2005). The broken and continuous thick lines represent the location of the IDT and a conducting plate, respectively.

The fluid (i.e. air or water) surrounding the sensor has an important roll on the wave propagation parameters such as velocity, amplitude and attenuation (White and Wenzel; 1988). Therefore, a change in the environment can be detected by measuring changes on the wave propagation. An overview of the mechanisms of interaction between sensors and overlaying fluids can be found in a review article by Lindner (2008). On the other hand, there are sensors that do not use the coupling of the wave with the surrounding fluid, but instead a sensing layer, which changes its mechanical properties (i.e. density) with the presence of a virus or a chemical substance (Figure 2.9), is utilized as a probe (Sivaramakrishnan et al., 2008; Moll et al., 2008). The change in the 
mechanical properties of the sensing layer produces a measurable change on the wave propagation characteristics.

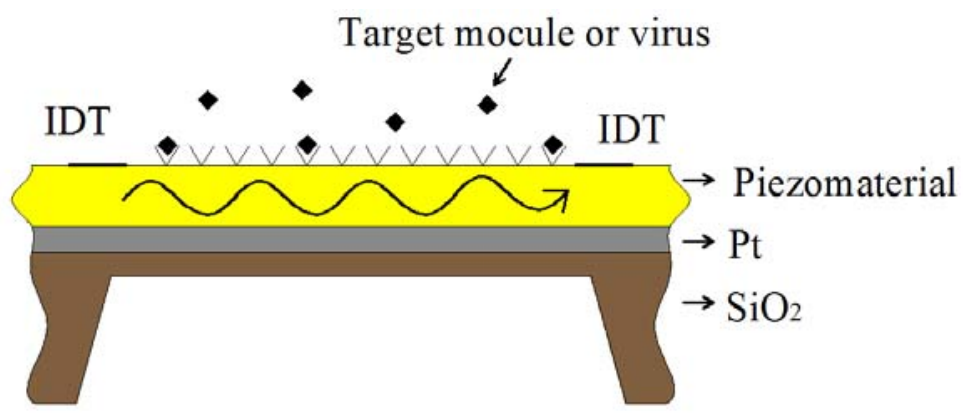

Figure 2.9. Ultrasonic sensor using a sensing layer to detect chemical molecules or viruses.

Sensors can be classified according the type of mechanical wave they generate. Rayleigh, Lamb and Love waves are commonly used for sensors. The displacements of Rayleigh and Love waves are confined to the surface of material; meanwhile the displacements of Lamb waves are contained between the two boundaries of a plate or diaphragm (Figure 2.10). Lamb wave sensors are characterized by larger surface displacements; therefore, the coupling with the surrounding fluid and sensitivity are higher. The choice between the type of wave depends mainly in the application; i.e. to measure the viscosity of fluids, sensors based on Love or shear Lamb waves are preferred.

(a)

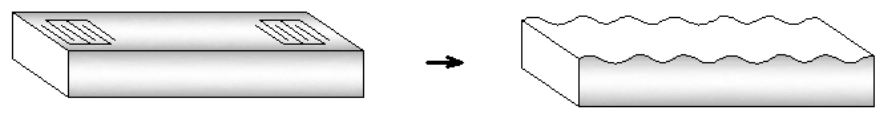

(b)

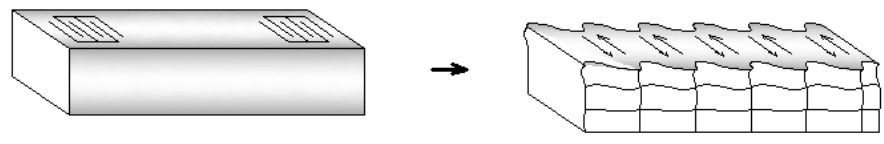

(c)

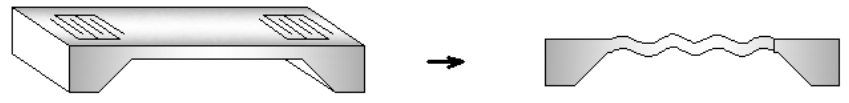

(d)

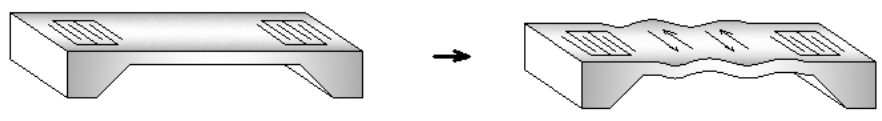

Figure 2.10. Types of mechanical waves used for acoustic sensors. (a) Rayleigh waves, (b) Love waves, (c) Symmetric and anti-symmetric (out of plane) Lamb waves and (d) Shear Lamb waves.

\subsubsection{Applications}

Surface acoustic sensors have been designed and used for a great variety of applications. This section does not intend to compile a complete summary of the 
applications; however some few applications, relevant to this study, will be highlighted. The applications can be divided according the sensing mechanism used by the device, i.e. solid-fluid coupling and sensing coating.

Sensors based on solid-fluid coupling are mainly used to monitor changes on the properties of the surrounding fluid. This type of sensors has been successfully applied to measure key electromechanical properties of the fluid such as density, viscosity, conductivity and permittivity. Density monitoring is one of the earliest and most common applications of acoustic sensors (White and Wenzel, 1988; Turton et al., 2006; Greenwood, 2006). The density of the surrounding fluid has an important effect on the wave propagation on plates, especially when the ratio between fluid to solid densities is high (Nayfeh and Chimenti, 1988). Sensors based on Love and Shear-Horizontal Lamb waves are the most suitable for viscosity sensing due to the in-plane displacements of the surface. The changes of the propagation parameters of these waves can be directly related to the viscosity of the fluid (Lec et al, 1988; Raimbault et al., 2008). On the other hand, changes of the electrical field in the surface of the sensor caused by a change of the conductivity of the fluid produce a measurable change in the wave propagation, due to the piezoelectric effect of the top layers (Kondoh and Shiokawa, 1995; Huang et al., 2001).

Another kind of sensor is based on sensing coatings bonded to the surface. In this case the change in propagation characteristics is not longer related to the electromechanical properties of the fluid, but instead with a change of the mechanical properties of the coating. This type of sensors enables to detect particles, chemical, proteins, bacteria, viruses present in the surrounding fluid. The number of application is vast, a review of some of these can be found in Lange et al., 2008; Caliendo et al., 1997; Hoummady et al., 1997.

\subsection{Elastic Guided Waves}

Single-element ultrasound transducers are usually modeled as infinite plates and 1D array transducer as finite-cross section plates. These assumptions are in accordance with the actual dimension of the transducers. For instance, the piezoelectric crystal of a single element transducer can be a disk of diameter $15 \mathrm{~mm}$ and a thickness of $150 \mu \mathrm{m}$. To optimally design transducers, the dynamic characteristics of these structures must be well understood.

Wave propagation is governed by the motion equation. The solution of this equation for certain boundary conditions results in a set of wave modes, usually called guided waves. The waves propagating on an infinite plate are usually known as Lamb waves. Three types of waves modes are found for Lamb waves: symmetric (S), antisymmetric (A) and in-plane shear (SH) modes (Fig. 2.11). One important characteristic of the propagation guided waves is that the wave velocity depends on the frequency; this effect is called dispersion behavior. The group velocity is usually associated with the energy propagation; i.e. if the group velocity is zero, there is not energy propagation and high-amplitude standing waves are present. 


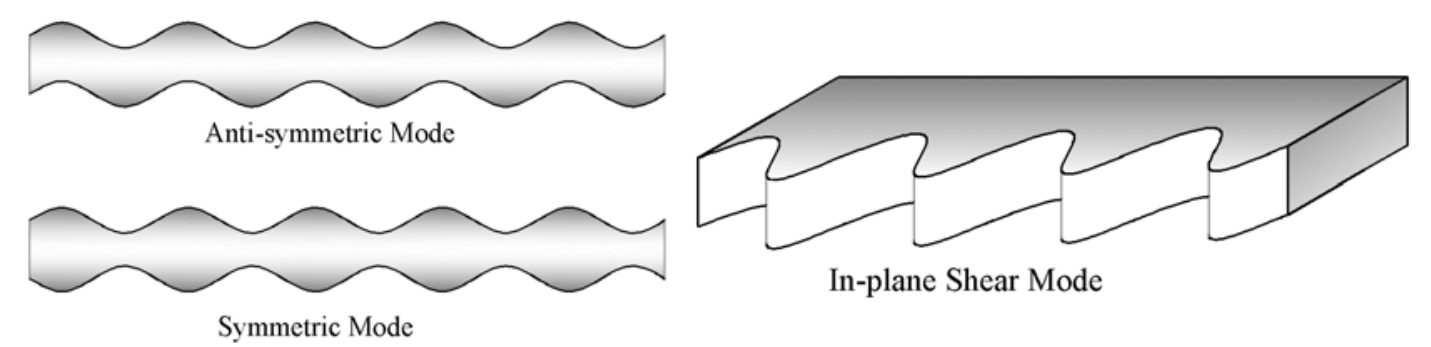

Figure 2.11. Anti-symmetric and Symmetric modes in Lamb waves.

Recent experimental studies (Clorennec et al., 2007; Gibson and Popovics, 2005; Holland and Chimenti, 2004) have shown that resonance for isotropic plates occur at frequencies where the group velocities of symmetric and anti-symmetric modes vanish. Since the group velocity of such modes vanishes, mechanical energy is trapped and high amplitude vibrations are present. The frequencies of the zero-group-velocity (ZGV) points depend on the materials properties and the thickness of the plate. The displacement spectrum for an impulse excitation shows the resonant peaks, from which the frequencies of the ZGV points can be obtained. These frequencies can be used to calculate the thickness or the Poisson's ratio for isotropic plates (Clorennec et al., 2007).

The ZGV point for the first-extensional $S_{1}$ mode has also been found useful for air-coupled ultrasonic imaging (Holland and Chimenti, 2004). In this case, the center frequency of the transducer is set to the frequency of ZGV point for the $S_{1}$ mode of the plate to be imaged. Color-scans of discontinuities in the thickness or mechanical properties can be obtained measuring the transmission efficiency in the region of interest. The same concept has been used to refine another NDT method called 'Impact Test' (Gibson and Popovics, 2005). In this, the normal displacements of a slab-like structure are measured after an impact is applied to the structure. The frequency spectra of these displacements are used to detect the first resonant frequency and then material properties or thickness can be calculated.

Knowing the dynamic characteristic of plates is important, especially for singleelement transducer design. Ultrasound transducers are designed to work at the resonance frequency of the piezoelectric element. Several analytic solutions for different materials and boundary conditions have been presented in the literature for infinite plates.

\subsubsection{Analytical Solutions for Infinite Plates}

Solutions of the vibration of plates have been of great interest of many researchers and used for many applications. Analytic solutions for infinite plates have been being presented for almost two centuries. In 1828, Poisson and Cauchy presented the solution for flexural vibrations at low frequency for isotropic plates. Of course, not all the published works on infinite plates can be referenced in this section; instead, a summary of some relatively recent analytic solutions for infinite plates is presented. Frequently, linear constitutive relations and small deformations are considered when modeling ultrasound transducers. Furthermore, fluid boundary condition has been considered as a subrogate of tissue due to the similarities on the acoustic properties. Hence, the references are restricted to those relevant to this research area. 
Solutions for isotropic materials surrounded by viscous (Zhu and $\mathrm{Wu}, 1995$ ) and invisid fluids ( $\mathrm{Zhu}$ and $\mathrm{Wu}, 1992$ ) have been presented to analyze the change on velocity with the frequency (dispersion behavior) and the attenuation of propagating modes. The solution for anisotropic plates with and arbitrary periodic loading was presented by (Mal, 1988). Piezoelectric homogeneous plates with stress-free boundary condition (Yang and Tsai, 2006), dielectric-fluid boundary condition (Yang and Chimenti, 1993; Yang and Lai, 2004) and conductive-fluid condition (Lee and Kuo, 2006; Yang and Shue, 2001) were studied to analyzed the dispersion behavior and the effect of fluid properties. Multilayered piezoelectric plates, including inversion layer, have been also analyzed (Powell et al., 1998; Huang et al., 2005).

Although analytical solutions have been proposed for a wide variety of cases, sometimes the geometry and/or the boundary conditions increase the mathematical complexity of the solution to such degree that numerical methods are required to obtain a solution. Finite Element Method (FEM) is a widely use technique to numerically analyze complicated structures. For instance, multi-layered and finite cross-section plates are usually analyzed through this method.

\subsubsection{Finite Element Modeling}

Finite element method (FEM) is frequently used when complicated geometries, loads and material are considered. For the analysis of guided waves, FEM can be divided in time-domain and frequency domain analyses. Both are used to obtain the dispersion behavior, resonance frequencies and other important characteristics of the piezoelectric structures. Time-domain FEM solutions are obtained by integration over time of the discretized motion equations. On the other hand, if Fourier transformation is applied to the discretized motion equations, the solution is obtained in terms of the frequency.

Time-domain schemes have been used to predict the performance of plates and ultrasound transducers (Zhou et al., 2006; McKeighen, 2001; Mills and Smith, 2002; Hossack and Hayward, 1991; Lerch, 1990). The effect of matching layers, backing layers, bonding layer and electrodes on the operation of the transducer can be addressed using this technique (Zhou et al., 2006; McKeighen, 2001). However, a large number of elements and simulation time are usually required.

Frequency-domain FEM has been broadly used to analyze piezoelectric structures and ultrasound transducers (Siao et al., 1994; Bai et al., 2004; Taciroglu et al., 2004; Pauley and Dong, 1975; Mukdadi et al., 2003, 2002). This method has been applied to calculate dispersion curves, group and phase velocity spectra for piezoelectric structures. Frequency-domain analysis usually offers more information than using time-domain; i.e. resonance frequencies can be easily identified. However, some boundary conditions, like fluid loading, are difficult to analysis in this domain.

1D and 2D arrays are usually periodic structures with periodic loading. Periodic boundary condition has been analyzed and implemented for FEM in both domains (Wilm et al., 2004, 2005; Ballandras et al., 2003, 2005; Predoi et al., 2007). Considering periodicity allows performing the analysis in a small representative region and obtaining results relevant to the entire structure. 


\section{Chapter Infinite Plate Model for Single Element Transducers}

3

The content of the chapter is based on the article published in International Journal of Solids and Structures 45, 5088-5102.

"Dispersion of elastic guided waves in piezoelectric infinite plates with inversion layer"

Daniel H. Cortes

Subhendu K. Datta

Osama M. Mukdadi 


\subsection{Finite Element Formulation}

Consider a layered piezoelectric plate as shown in Fig. 3.1. The piezoelectric plate consists of $N$ parallel, homogeneous, and anisotropic layers, which are perfectly bonded together. A global rectangular coordinate system $(X, Y, Z)$ is adopted such that the $Z$-axis coincides with the thickness direction. The $X$ and $Y$ axes are parallel to the infinite direction of the plate. We adopt a hybrid analytical-numerical approach to model elastic guided wave propagation in layered anisotropic infinite piezoelectric plates. The analysis couples analytical treatment of the motion in the $X Y$ plane with a numerical treatment of the motion along the thickness direction of the plate using the finite-element method. To effect the latter, we discretize the thickness (Z-axis) of the plate using threenode finite-elements, each of which has associated with it a local coordinate system $(x, y$, $z$ ), which is parallel to the global coordinate system. The displacement and electric potential are assumed to be time-dependent and functions of local coordinates system ( $x$, $y, z$ ). Since the displacements are small compared to the thickness, a linear stress-strainelectrical field will be used to describe the dynamic behavior of the plate. The constitutive equation in the matrix form is given by

$$
\mathbf{Q}=\mathbf{C}^{*} \mathbf{q}
$$

where $\mathbf{Q}=\left[T_{x x}, T_{y y}, T_{z z}, T_{y z}, T_{x z}, T_{x y}, D_{x}, D_{y}, D_{z}\right]$ is a vector of the stress components and the electric displacements, $\mathbf{q}=\left[S_{x x}, S_{y y}, S_{z z}, 2 S_{y z}, 2 S_{x z}, 2 S_{x y}, E_{x}, E_{y}, E_{z}\right]$ is a vector with the components of the mechanical strain and electric field components, and $\mathbf{C}^{*}$ is a matrix containing the elastic constants $\mathbf{c}$, dielectric constants $\varepsilon$ and the piezoelectric stress constants e:

$$
\mathbf{C}^{*}=\left[\begin{array}{cc}
\mathbf{c} & -\mathbf{e}^{T} \\
\mathbf{e} & \boldsymbol{\varepsilon}
\end{array}\right] .
$$

Without loss of generality, we assume that the waves are propagating in the $x$ direction. The kinematic equation relates the vector $\mathbf{q}$ to the displacements and electric potential vector $\mathbf{v}=\left[u_{x}, u_{y}, u_{z}, \phi\right]$ as,

$$
\mathbf{q}=\mathbf{B}_{1} \mathbf{v}_{, x}^{e}+\mathbf{B}_{2} \mathbf{v}^{e}
$$

where $\mathbf{B}_{1}$ and $\mathbf{B}_{2}$ are operator matrices defined in appendix A.

The equation of motion can be obtained using the Hamilton's principle (Tiersten, 1969):

$$
\delta \int_{t_{0}}^{t_{1}}(K E-H+W) d t=0
$$

where $K E, H$ and $W$ are the kinetic energy, electrical enthalpy, and potential energy of the prescribed surface forces and charges, respectively. These terms can be expressed as:

$$
K E=\frac{1}{2} \iiint_{v} \dot{\mathbf{v}}^{T} \boldsymbol{\rho} \dot{\mathbf{v}} d v, H=\frac{1}{2} \iiint_{v} \mathbf{q}^{T} \mathbf{C} \mathbf{q} d v, \text { and } W=\iint_{S} \mathbf{v}^{T} \mathbf{f} d S,
$$


where, $\quad \mathbf{C}=\left[\begin{array}{cc}\mathbf{c} & -\mathbf{e}^{T} \\ -\mathbf{e} & -\mathbf{\varepsilon}\end{array}\right], \quad$ and $\quad \boldsymbol{\rho}=\left[\begin{array}{llll}\rho & & & \\ & & \rho & \\ & & \rho & \\ & & 0\end{array}\right]$.

The thickness direction (z-direction, Fig. 3.1) of the plate is discretized using three-node elements. Within each sublayer, quadratic interpolation functions with three equally spaced nodal surfaces are used. The explicit form of these interpolations over a sublayer is shown in Appendix A.

The field variables $\mathbf{v}$ can be written in terms of the vector of nodal variables $\mathbf{v}^{e}$ as follows:

$$
\mathbf{v}(x, z, t)=\mathbf{N}(z) \mathbf{v}^{e}(x, t),
$$

where $\mathbf{N}$ is a matrix composed of the interpolation functions. Eq. (3.4) can rewritten in terms of Eq. (3.6) and Eq. (3.5) as:

$$
\delta \int_{t_{0}}^{t_{1}}\left[\frac{1}{2} \int\left(\dot{\mathbf{v}}^{e T} \mathbf{m}^{e} \dot{\mathbf{v}}^{e}-\mathbf{v}^{e T}{ }_{, x} \mathbf{k}_{11}^{e} \mathbf{v}_{, x}^{e}-\mathbf{v}_{, x}^{e T} \mathbf{k}_{12}^{e} \mathbf{v}^{e}-\mathbf{v}^{e T} \mathbf{k}_{21}^{e} \mathbf{v}_{, x}^{e}-\mathbf{v}^{e T} \mathbf{k}_{22}^{e} \mathbf{v}^{e}-\mathbf{v}^{e T} \mathbf{f}^{e}\right) d x\right] d t=0 .
$$

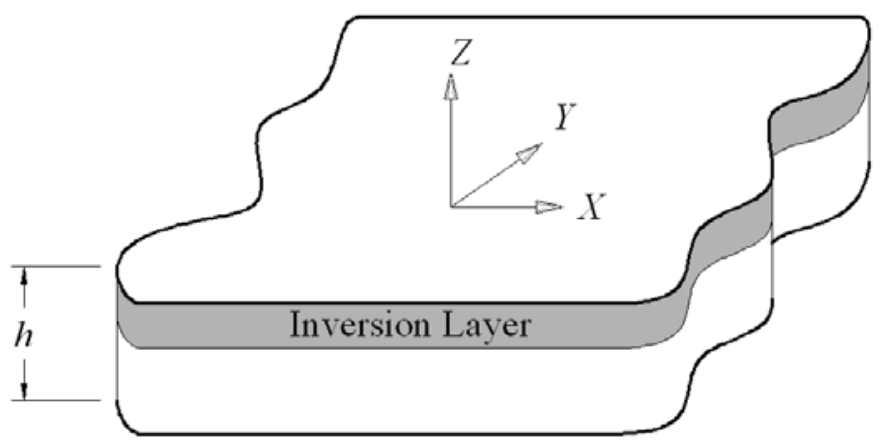

Figure 3.1. Coordinate system and inversion layer distribution.

where $\mathbf{k}_{11}^{e}, \mathbf{k}_{12}^{e}, \mathbf{k}_{21}^{e}$ and $\mathbf{k}_{22}^{e}$ are element stiffness matrices, $\mathbf{m}^{e}$ is the element mass matrix and $\mathbf{f}^{e}$ is a vector of nodal forces (see Appendix A for details). The global system of equations can be obtaining after assembling the element matrices and carrying out the variation of Eq. (3.7):

$$
\mathbf{M} \ddot{\mathbf{V}}+\mathbf{K}_{1} \mathbf{V}+\mathbf{K}_{2} \mathbf{V}_{, X}-\mathbf{K}_{3} \mathbf{V}_{, X X}=\mathbf{F}
$$

To solve Eq. (3.8), a semi-analytical technique will be implemented in the following section. In this study, we consider open circuit $\left(D_{Z}=0\right)$ and traction-free $\left(T_{X Z}=T_{Z Z}=0\right)$ boundary conditions on the upper and lower surfaces of the plate. Note that Eq. (3.8) is the semi - analytical finite element equation, which is obtained by discretizing in the $Z$ direction. This equation can now be solved by using standard Fourier transforms techniques in $X$ and $t$.

Define the Fourier transformation as

$$
\tilde{\Phi}(k, \omega)=\int_{-\infty}^{\infty} \int_{-\infty}^{\infty} \Phi(X, t) e^{-i(k X-\omega t)} d X d t,
$$


where $\Phi(X, t)$ is a finite function, $k$ and $\omega$ represent the wave numbers along $X$ direction and the angular frequency, respectively. Applying this transformation to Eq. (3.8), the differential equation is transformed into:

$$
\left(\mathbf{K}_{1}-i k \mathbf{K}_{2}+k^{2} \mathbf{K}_{3}-\omega^{2} \mathbf{M}\right) \tilde{\mathbf{V}}=\tilde{\mathbf{F}} .
$$

The dispersion relations of the propagating modes are found by solving the eigenvalue problem arises when considering the homogeneous case $(\tilde{\mathbf{F}}=\mathbf{0})$ of Eq. (3.10). If the wavenumber $k$ is defined, then equations (3.10) can be solved for the angular frequencies $\omega$ of the propagating modes. The phase and group velocities can be expressed, respectively, as,

$$
\mathrm{v}_{\mathrm{p}}=\frac{\omega}{k}, \text { and } \mathrm{v}_{\mathrm{g}}=\frac{\partial \omega}{\partial k} .
$$

Dispersion curves showing the dependence of the phase and group velocities on the angular frequencies can be obtained using Eqs. (3.10) and (3.11).

\subsection{Electro-Elastodynamic Green's Functions}

Solving the homogenous part of Eq. (3.10) will yield the dispersion relation for elastic guided waves in infinite piezoelectric plates. To derive the Green's functions, one can rewrite Eq. (3.10) including the forcing term in the form (Mukdadi and Datta, 2003),

$$
[\mathbf{A}] \mathbf{U}=\gamma[\mathbf{B}] \mathbf{U}+\mathbf{P},
$$

where

and

$$
\begin{array}{rlrl}
\mathbf{A} & =\left[\begin{array}{cc}
\mathbf{0} & \mathbf{I} \\
\mathbf{K}_{1}-\omega^{2} \mathbf{M} & -\mathbf{K}_{2}
\end{array}\right], & \mathbf{B}=\left[\begin{array}{cc}
\mathbf{I} & \mathbf{0} \\
\mathbf{0} & \mathbf{K}_{3}
\end{array}\right], \\
\mathbf{U}=\left[\begin{array}{lll}
\tilde{\mathbf{V}} & \gamma \tilde{\mathbf{V}}
\end{array}\right]^{T}, & \mathbf{P}=\left[\begin{array}{cc}
\mathbf{0} & \tilde{\mathbf{F}}
\end{array}\right]^{T}, \text { and } \gamma=i k .
\end{array}
$$

Note that matrix $\mathbf{A}$ is not symmetric but $\mathbf{B}$ is symmetric. The homogenous part of Eq. (3.12) will yield the characteristic equation, the roots of which (eigenvalues) are the wavenumbers of the modes for a given frequency $\omega$. These wavenumbers can be real, imaginary or complex numbers. The imaginary and complex eigenvalues correspond to the evanescent modes, while real values are for the propagating modes.

The right and left eigenvectors, $\varphi_{m}$ and $\psi_{m}$ respectively, associated with each eigenvalue can be found solving the systems of equations (written in an abbreviated form)

$$
\left[\mathbf{A}-\gamma_{m} \mathbf{B}\right] \varphi_{m}=\mathbf{0}, \text { and }\left[\mathbf{A}^{T}-\gamma_{m} \mathbf{B}\right] \psi_{m}=\mathbf{0} .
$$

Here, $\varphi_{m}$ and $\psi_{m}$ satisfy the bi-orthogonality relations:

$$
\psi_{m}^{T} \mathbf{B} \varphi_{m}=\delta_{m n} B_{n}, \quad \text { and } \quad \psi_{m}^{T} \mathbf{A} \varphi_{m}=\delta_{m n} \gamma_{m} B_{n} .
$$

The eigenvectors can be combined as the upper and lower parts to give

$$
\varphi_{m}=\left\{\begin{array}{c}
\varphi_{m u} \\
\varphi_{m l}
\end{array}\right\}=\left\{\begin{array}{c}
\varphi_{m u} \\
\gamma_{m} \varphi_{m l}
\end{array}\right\}, \psi_{m}=\left\{\begin{array}{l}
\psi_{m u} \\
\psi_{m l}
\end{array}\right\}=\left\{\begin{array}{c}
\psi_{m u} \\
\gamma_{m} \psi_{m l}
\end{array}\right\} .
$$


These eigenvectors can be used to calculate the response spectrum due to electrical or mechanical excitations. The solution of Eq. (3.12) is the weighted sum of all modes:

$$
\mathbf{U}(\gamma, \omega)=\sum_{m=1}^{2 N} \bar{U}_{m} \varphi_{m} .
$$

Inserting Eq. (3.17) into Eq. (3.12), the following system of equations is obtained:

$$
[\mathbf{A}-\gamma \mathbf{B}] \sum_{m=1}^{2 N} \bar{U}_{m} \varphi_{m}=\mathbf{P} .
$$

Multiplying Eq. (3.18) on the left side by $\psi_{m}^{T}$ and applying the bi-orthogonality relations, the $\bar{U}_{m}$ coefficients can be obtained:

$$
\bar{U}_{m}=\frac{\psi_{m}^{T} \mathbf{P}}{\left(\gamma_{m}-\gamma\right) B_{m}} .
$$

In terms of the lower and upper eigenvectors (Eq. 3.15), we expressed the resulting Green's functions in the wavenumber-frequency domain by substituting $\bar{U}_{m}$ in Eq. (3.17) as follows,

$$
\tilde{\mathbf{V}}(\gamma, \omega)=\sum_{m=1}^{2 N} \frac{\gamma_{m} \psi_{m u}^{T} \tilde{\mathbf{F}}}{\left(\gamma_{m}-\gamma\right) B_{m}} \varphi_{m u} .
$$

The displacement and electrical potential in the space-frequency domain were determined by applying the inverse Fourier transform, which was evaluated numerically using Cauchy's residue theorem,

$$
\hat{\mathbf{V}}(X ; \omega)=-i \sum_{m=1}^{N} \frac{\gamma_{m} \psi_{m u}^{T} \tilde{\mathbf{F}}}{B_{m}} \varphi_{m u} e^{-\gamma_{m} X}
$$

Equation (3.21) represents the displacement and electrical potential Green's functions in the space-frequency domain due to force or electric excitation.

\subsection{Frequency Response Due to Impulse Loading}

To obtain ultrasound images with optimum axial and lateral spatial resolutions, impulsive loads are usually used with broadband ultrasound transducers. In this study, we aim to study the resonance modes of elastic guided waves for harmonic ultrasound transducer design. To do so, we considered an impulsive line load applied to the surface of the piezoelectric plate. This line load along the $Y$-axis is acting into the thickness direction of the plate ( $Z$-axis). This impulse line load can be expressed in the form,

$$
\mathbf{F}(X, t)=\delta(X) \delta(t) \mathbf{F}_{0},
$$

where $\mathbf{F}_{0}$ is a vector with all the components equal to zero, except one component corresponding to force acting into $Z$-axis or charge applied on the piezoelectric plate. Applying the Fourier transform (Eq. 3.9) to Eq. (3.22) results in

$$
\tilde{\mathbf{F}}(k, \omega)=\mathbf{F}_{0} \text {. }
$$

The frequency spectrum of the normal displacements and electrical potential can be expressed, using Eq. (3.21), as: 


$$
\hat{\mathbf{V}}(X ; \omega)=-i \sum_{m=1}^{N} \frac{\gamma_{m} \psi_{m u}^{T} \mathbf{F}_{0}}{B_{m}} \varphi_{m u} e^{-\gamma_{m} X} .
$$

The above equation represents the frequency response of the displacement and electrical potential at the point $X$ due to a line force along the $Y$-axis and acting in the Zdirection. The numerical results illustrated in the following section will consider the frequency spectrum of the normal displacement calculated at the same point of excitation (i.e. $X=0$ ). This is analogous to the experiment reported by Clorennec et al., (2007).

\subsection{Numerical Results and Discussion}

Equations (3.10) and (3.24) govern the dispersion behavior and the frequency spectrum of displacements and electrical potential of guided wave propagation in layered piezoelectric infinite plates. In this study, numerical results for single layer and bilayered plates used for fabricating single-element medical ultrasound transducers will be addressed.

Validation of the SAFE method with experiment was made by comparison of numerical results for a $0.9 \mathrm{~mm}$ steel plate and experimental results reported by Clorennec et al., (2007). The mechanical properties used for steel are shown in Table 3.1a. The dispersion curves for a steel plate of $0.9 \mathrm{~mm}$ show two ZGV points at 3.08 and $5.36 \mathrm{MHz}$ (Fig. 3.2a), corresponding to the first extensional $\left(\mathrm{S}_{1}\right)$ and second flexural $\left(\mathrm{A}_{2}\right)$ modes. Excellent agreement for the peaks and off-peak values is observed between the frequency spectra of the normal displacement obtained by this method and experimental results (Fig. 3.2b). A comparison between the dispersion curves and the frequency spectra of the normal displacement clearly show that resonance peaks correspond to ZGV frequency points. This phenomenon shall be useful for piezoelectric resonators and ultrasound transducers used for imaging. Note that even though the experiment was for a point load, the frequency dependence of the displacement is the same for the point and line loads (except for a normalization factor). This is because the dispersion behavior is the same in two and three dimensions for an isotropic plate.

a)

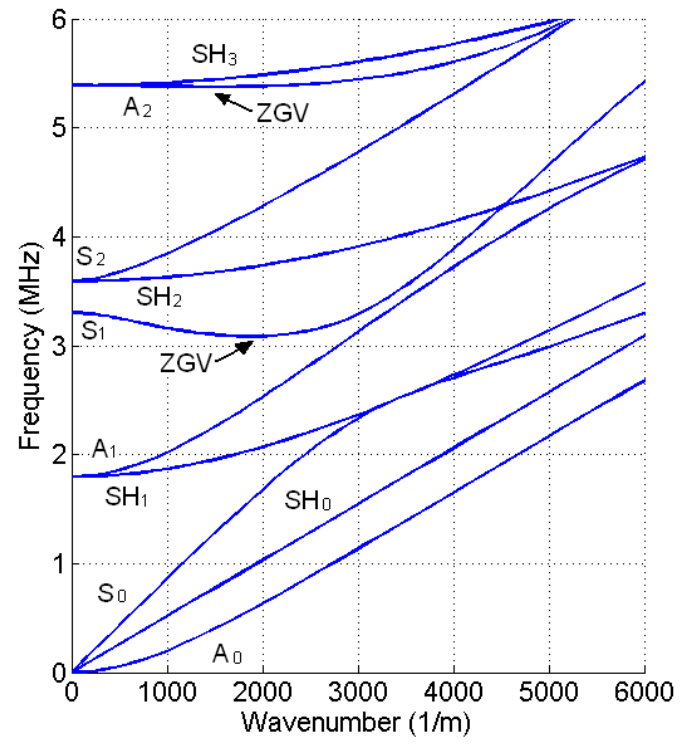

b)

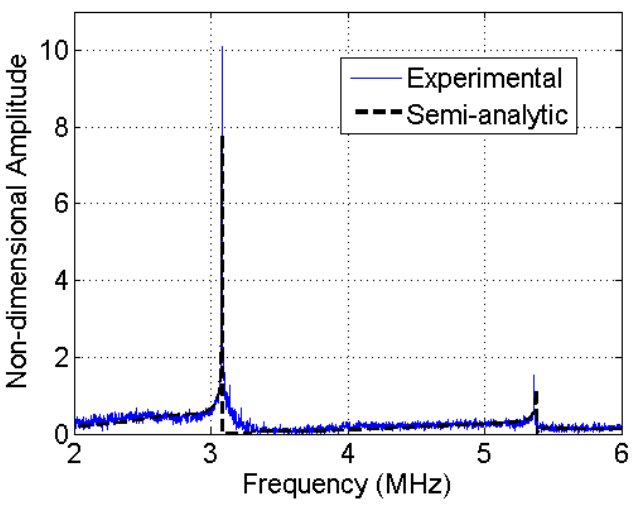


Figure 3.2. Dynamic characteristics of a steel plate of $0.9 \mathrm{~mm}$ thick: a) dispersion curves, b) displacement spectrum.

Figure 3.3 shows the dispersion curves and the frequency spectra of the normal displacement of a $36^{\circ}$ rotated $y$-cut $\mathrm{LiNbO}_{3}$ layered plate of $0.5 \mathrm{~mm}$ total thickness. The effect of the ratio of the thickness of the inversion layer (IL) to the total thickness of the plate was examined. The mechanical and piezoelectric properties are shown in Tables $3.1 \mathrm{a}$ and $3.1 \mathrm{~b}$, respectively. The wavenumber was normalized by multiplying with the thickness $(h)$ and the non-dimensional frequency is defined as $\omega / \omega_{0}$ where by $\omega_{0}=\sqrt{C_{44} / \rho} / h$. Here $C_{44}$ is an elastic constant and $\rho$ the mass density. The dispersion curves (Fig. 3.3a) for several ratios $(0,30$ and $50 \%)$ of IL thickness to the plate show negligible differences for shear-horizontal modes $\mathrm{SH}_{0}, \mathrm{SH}_{2}$ and $\mathrm{SH}_{3}$. For the first-order shear-horizontal $\mathrm{SH}_{1}$ mode, the curves are identical for $k$ in the range $0-2.5$, but after that, small differences are seen for the three percentages of IL thickness ratios. Small differences are observed for the $\mathrm{S}_{0}, \mathrm{~S}_{1}$ and $\mathrm{S}_{2}$ modes. The cut-off frequencies for the $\mathrm{SH}_{1}$ and $\mathrm{A}_{1}$ modes show small differences for $0 \%$ and $30 \%$ of IL, but are the same for $50 \%$ thickness ratio of IL. On the other hand, for $\mathrm{SH}_{2}$ and $\mathrm{S}_{2}$ modes, the cut-off frequencies are slightly different for $30 \%$ and $50 \%$ thickness ratios of IL. However, these cut-off frequencies for $\mathrm{SH}_{2}$ and $\mathrm{S}_{2}$ modes are equal for single $\mathrm{LiNbO}_{3}$ plate without inversion layer (i.e., $0 \%$ IL thickness ratio). The $\mathrm{ZGV}$ point for the $\mathrm{S}_{1}$ mode does not change considerably. However, the lowest frequency for this ZGV point corresponds to $0 \%$ IL thickness ratio. On the other hand, the lowest frequency for the ZGV point corresponding to the $\mathrm{A}_{2}$ mode is obtained for $50 \%$ IL thickness ratio.

Table 3.1a. Mechanical properties and mass densities.

\begin{tabular}{|c|c|c|c|c|c|c|c|c|}
\hline & \multicolumn{7}{|c|}{ Elastic Properties (GPa) } & \multirow{2}{*}{$\begin{array}{c}\text { Density } \\
\left(\mathrm{Kg} / \mathrm{m}^{3}\right) \\
\rho\end{array}$} \\
\hline & $C_{11}$ & $C_{12}$ & $C_{13}$ & $C_{14}$ & $C_{33}$ & $C_{44}$ & $C_{66}$ & \\
\hline Steel & 277.5 & 113.4 & 113.4 & 0 & 277.5 & 82 & 82 & 7850 \\
\hline $\mathrm{LiNbO}_{3}$ & 199 & 51.85 & 70.85 & 7.83 & 234.18 & 59.85 & 72.09 & 4640 \\
\hline PZT-4 & 139 & 77.8 & 74.3 & 0 & 115 & 25.6 & 30.6 & 7500 \\
\hline
\end{tabular}

Table 3.1b. Piezoelectric properties.

\begin{tabular}{l|ccccc}
\hline & \multicolumn{3}{|c}{$\begin{array}{c}\text { Piezoelectric Stress Constant } \\
\left(\mathrm{C} / \mathrm{m}^{2}\right)\end{array}$} & \multicolumn{2}{c}{ Clamped dielectric } \\
constants
\end{tabular}



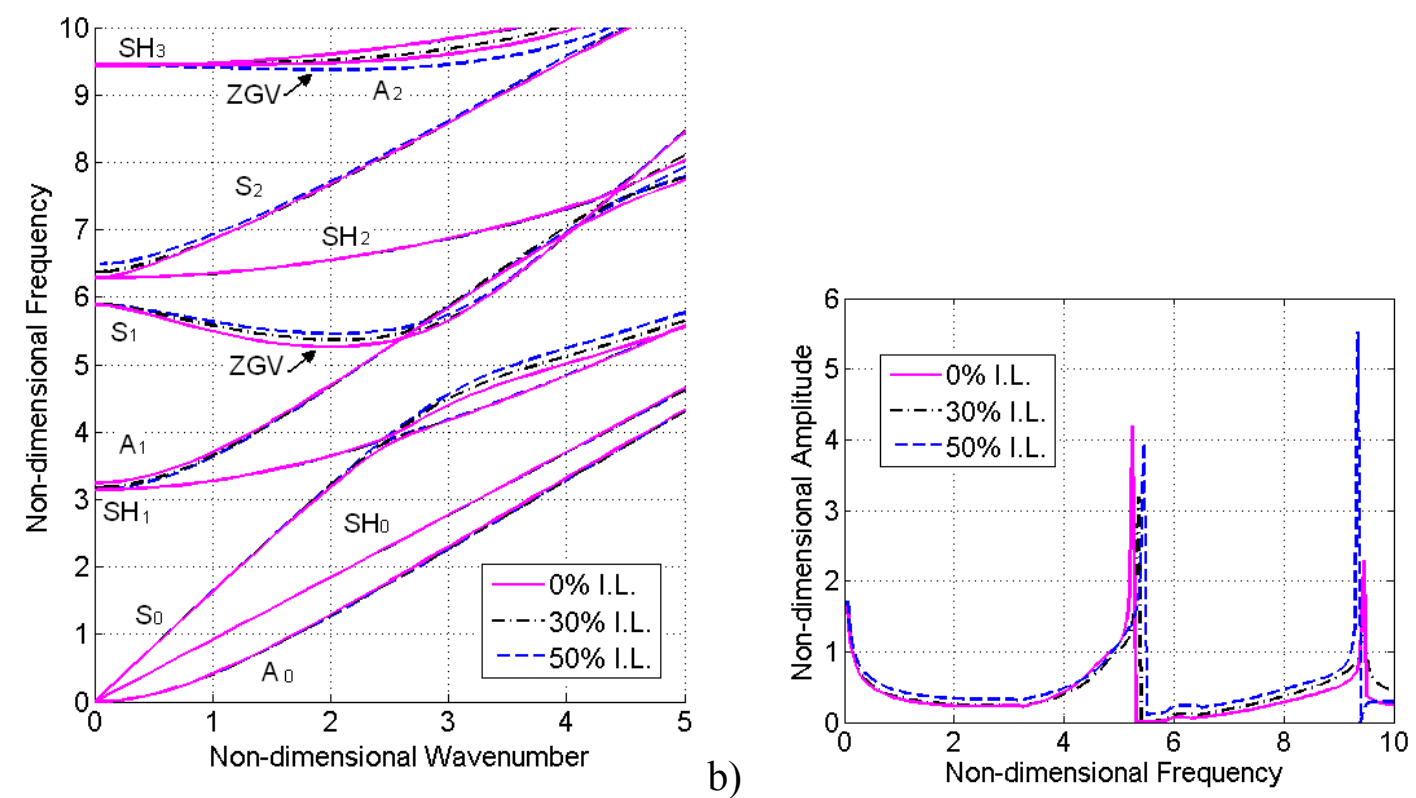

Figure 3.3. Vibrational behavior of $36^{\circ}$ rotated $y$-cut $\mathrm{LiNbO}_{3}$ plate for several percentages of inversion layer and $0.5 \mathrm{~mm}$ thickness: a) Dispersion Curves, b) Displacement Spectrum

A similar analysis of the dispersion curves (Fig. 3.4a) for PZT-4 (Table 3.1) shows that the shear-horizontal $\mathrm{SH}_{0}, \mathrm{SH}_{1}, \mathrm{SH}_{2}$, and $\mathrm{SH}_{3}$ modes are similar for all the ratios of IL analyzed. A considerable difference is observed for the cut-off frequencies of the $A_{1}$ mode, especially for $0 \%$ IL thickness ratio. In a similar way, the cut-off frequencies for $\mathrm{S}_{1}$ modes show considerable differences. It is noted that, the cut-off frequencies of $\mathrm{S}_{1}$ and $\mathrm{SH}_{2}$ modes are the same for $0 \%$ IL thickness ratio. The same is found to be true for the $\mathrm{S}_{1}$ and $\mathrm{S}_{2}$ for $50 \%$ of IL. A very important observation is that for this material the second $\mathrm{ZGV}$ point is not present on $\mathrm{A}_{2}$ mode dispersion curve, but is found on that of $\mathrm{S}_{4}$. Negish et al. (1987) showed that for isotropic materials a ZGV point for the $A_{2}$ mode exists only when the Poisson's ratio $(v)$ is less than 0.31. Furthermore, $\mathrm{ZGV}$ points can be also found for $\mathrm{S}_{3}, \mathrm{~S}_{4}, \mathrm{~S}_{6}$ and $\mathrm{A}_{5}$ modes for specific values of $v$. In fact, there is not an explicit relation between the ZGV points and the elastic constants for anisotropic materials. 


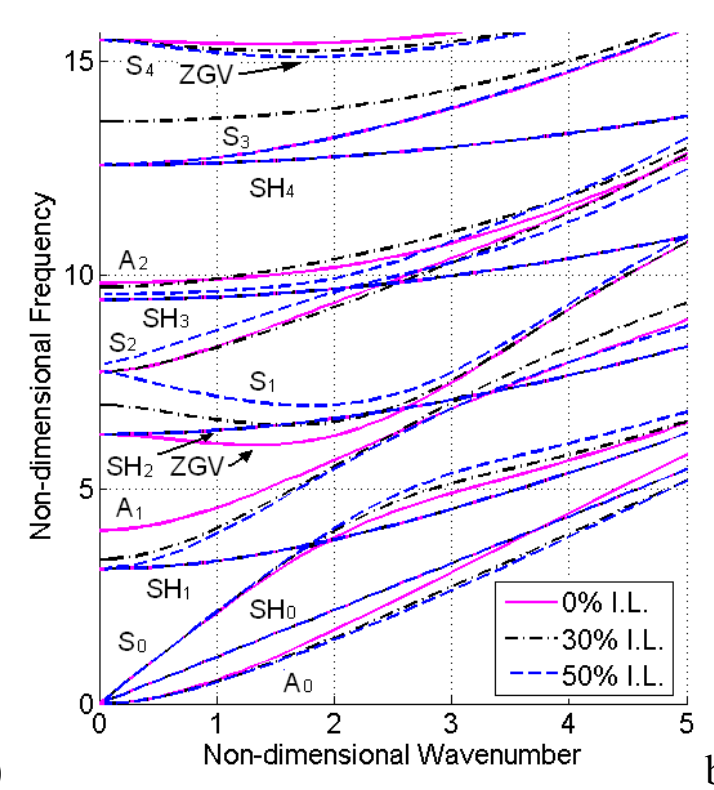

a)

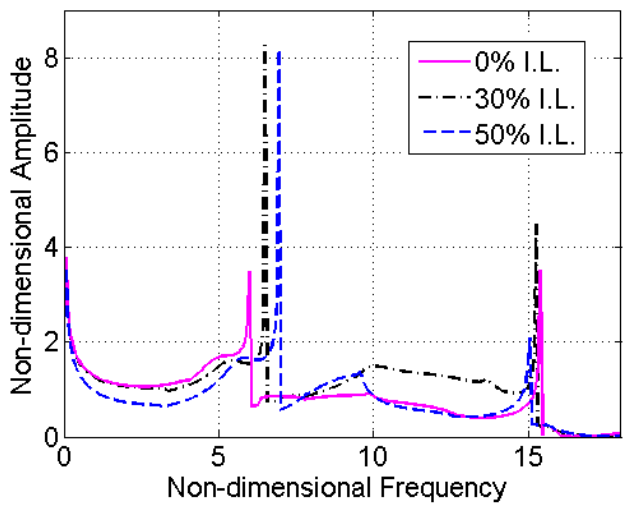

Figure 3.4. Vibrational behavior of PZT-4 plate for several percentages of inversion layer and 0.5mm thickness: a) Dispersion Curves, b) Displacement Spectrum.

Figures $3.3 \mathrm{~b}$ and $3.4 \mathrm{~b}$ clearly show that the resonant frequencies for the plates correspond to the $\mathrm{ZGV}$ points on the dispersion curves. For $\mathrm{LiNbO}_{3}$, the first two resonant peaks correspond to the $\mathrm{ZGV}$ of $\mathrm{S}_{1}$ and $\mathrm{A}_{2}$ modes. In the same way, for PZT-4, the first two resonant peaks are obtained for the $Z G V$ points of $S_{1}$ and $S_{4}$ modes. The change in the frequency for the resonant peaks with the change in percentage of IL is small, except for first resonant peak for PZT-4. In this case, the resonant frequency was shifted from $\omega / \omega_{0}=6.0$ to $\omega / \omega_{0}=6.95$. Small peaks are observed at the cut-off frequency for the $\mathrm{A}_{2}$ mode, especially for $50 \%$ IL thickness ratio. A comparison between the resonant frequencies obtained using the SAFE method presented here (Table 3.2) shows that the second resonant peak for PZT-4 with 50\% IL thickness ratio is located at almost twice the frequency of the first peak, making this configuration optimum for harmonic imaging. Additionally, there is no significant difference in the ratio of the first two resonant frequencies of $\mathrm{LiNbO}_{3}$ when the percentage of IL thickness ratio is changed.

Table 3.2. Comparison of the resonance frequencies for several percentages of IL thickness ratio using SAFE method.

\begin{tabular}{c|cccc}
\hline & $I L$ & $1^{\text {st }} f_{\text {SAFE }}\left(\omega / \omega_{0}\right)$ & $2^{\text {nd }} f_{\text {SAFE }}\left(\omega / \omega_{0}\right)$ & $2^{\text {nd }} f_{\text {SAFE }} / 1^{\text {st }} f_{\text {SAFE }}$ \\
\hline \multirow{3}{*}{$\mathrm{LiNbO}_{3}$} & $0 \%$ & 5.25 & 9.45 & 1.78 \\
& $30 \%$ & 5.34 & 9.44 & 1.77 \\
& $50 \%$ & 5.46 & 9.35 & 1.71 \\
\hline \multirow{3}{*}{ PZT-4 } & $0 \%$ & 6.00 & 15.40 & 2.57 \\
& $30 \%$ & 6.50 & 15.25 & 2.35 \\
& $50 \%$ & 6.95 & 15.05 & 2.16 \\
\hline
\end{tabular}


a)

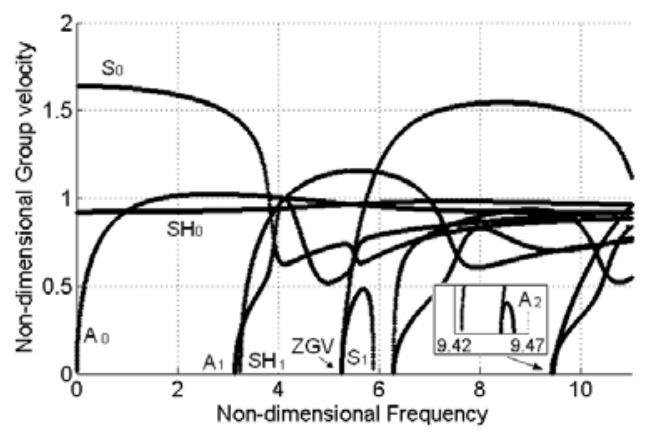

c)

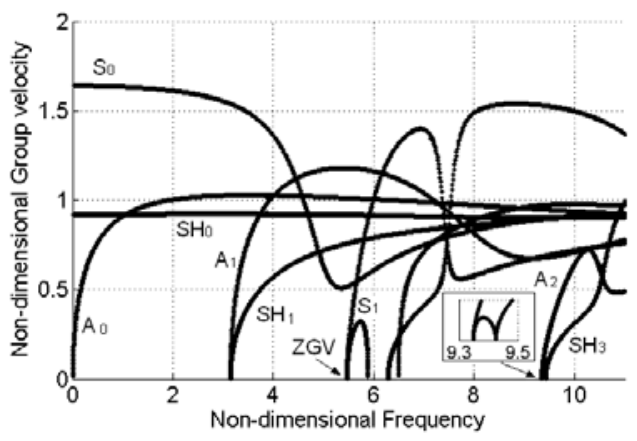

b)

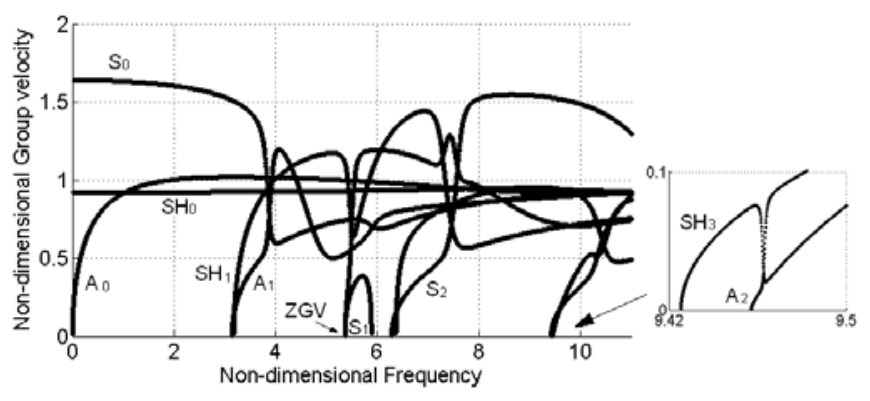

Figure 3.5. Group velocity spectrum for $36^{\circ} y$-cut $\mathrm{LiNbO}_{3}$ : a) $0 \%$, b) $30 \%$, and c) $50 \%$ IL thickness ratios.

Figure 3.5 shows the frequency dependence of the non-dimensional group velocity $\left(\overline{\mathrm{v}}_{g}=\mathrm{v}_{g} / \sqrt{C_{44} / \rho}\right)$ for $\mathrm{LiNbO}_{3}$. The group velocities were calculated using Eq. (3.11). It is clearly seen that for all percentages of inversion layer there is a ZGV point for the $S_{1}$ mode below the cut-off frequency. However, there is no $Z G V$ point for the second anti-symmetric $\mathrm{A}_{2}$ mode with $30 \%$ IL thickness ratio, instead a crossing between the $\mathrm{A}_{2}$ and $\mathrm{SH}_{3}$ modes is observed. Figure 3.6 shows the group velocity dispersion curves for PZT-4. The ZGV points for $\mathrm{S}_{1}$ and $\mathrm{S}_{4}$ modes are observed for all thickness ratios of IL. These results show strong influence of the piezoelectric material anisotropy on the ZGV modes, resonance characteristics and dispersion behavior of layered piezoelectric plates used for ultrasound imaging transducers. These theoretical findings provide a means for optimizing resonance characteristics of piezoelectric resonators.

In the approximate conventional methods (KLM) for transducer design the resonance frequency is defined as $f_{0}=C_{d} / 2 h$, where $C_{d}=\sqrt{C_{33} / \rho}$ is the bulk dilatational speed of the piezoelectrical material and $h$ the thickness. A comparison of the first and second resonance frequencies obtained using this method and the one proposed here for $0 \%$ IL thickness ratio is shown in Table 3.3. The error in calculating the first resonance for $36^{\circ}$ rotated $y$-cut $\mathrm{LiNbO}_{3}$ and PZT-4 is about $10 \%$. For the second resonant frequencies the errors are considerably high for both materials (about 17\%). These differences indicate that the resonant frequencies are strongly influenced by the material properties and thus should be carefully obtained specially when designing multi-resonant transducers for harmonic imaging. 
a)

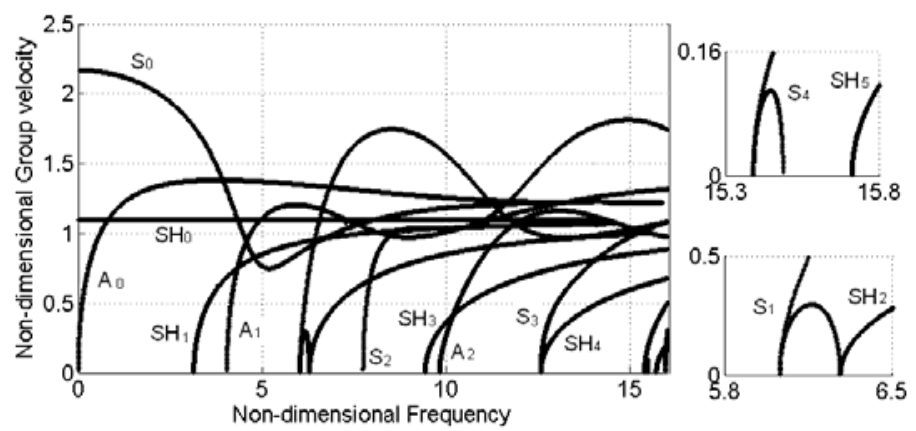

b)

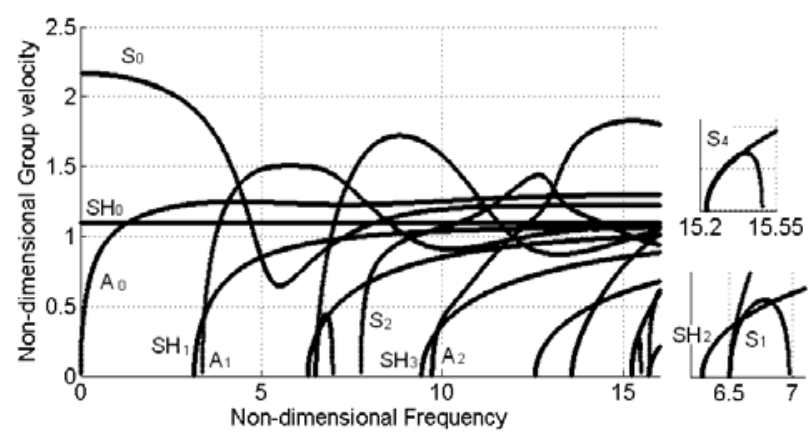

c)

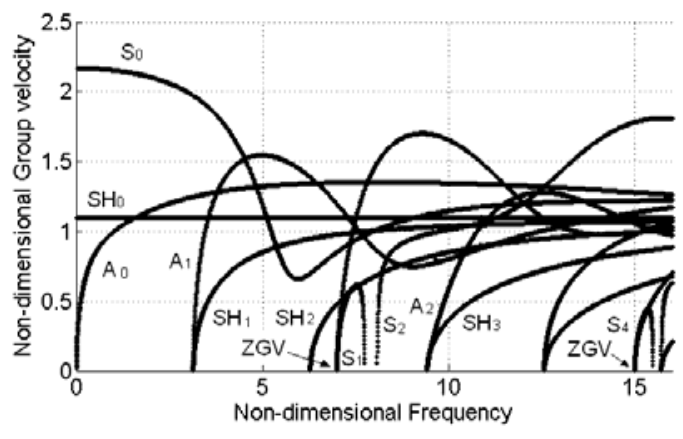

Figure 3.6. Group velocity spectrum for PZT-4: a) 0\%, b) $30 \%$, and c) $50 \%$ IL thickness ratios.

Table 3.3. Comparison of the resonance frequencies calculated using the KLM approximation $\left(f_{0}=c / 2 d\right)$ and using SAFE method.

\begin{tabular}{l|cccccc}
\hline & $\begin{array}{c}f_{0}=c / 2 d \\
(M H z)\end{array}$ & $\begin{array}{c}f_{\text {SAFE }} \\
(M H z)\end{array}$ & $f_{\text {SAFE }} / f_{0}$ & $\begin{array}{c}2 f_{0} \\
(M H z)\end{array}$ & $\begin{array}{c}2^{\text {nd }} f_{\text {SAFE }} \\
(M H z)\end{array}$ & $2^{\text {nd }} f_{\text {SAFE }} / 2 f_{0}$ \\
\hline $\mathrm{LiNbO}_{3}$ & 6.63 & 6.57 & 0.984 & 13.26 & 11.75 & 0.886 \\
$\mathrm{PZT}-4$ & 3.91 & 3.52 & 0.901 & 7.82 & 9.05 & 1.157 \\
\hline
\end{tabular}

Recent studies have shown the improvement in the bandwidth for transducers made of $\mathrm{LiNbO}_{3}$ when $30 \%$ IL thickness ratio has been included (Zhou et al., 2005, 2006; Nakamura et al., 2003; 2005). Zhou et al (2006) showed that the bandwidth can be increased up to $90 \%$ using IL and a proper selection of the matching layers. Nakamura et al (2003) found that the transducer characteristics depend on whether the IL is in the front (upper) or back (lower) sides of the transducer. However, in both cases the total efficiency of the transducer was improved at $f_{0}$ and $2 f_{0}$. 


\subsection{Concluding Remarks}

A semi-analytical finite element method has been used to theoretically predict the resonant frequencies and the dispersion behavior of layered piezoelectric plates. The analysis of piezoelectric layered plates shows that the resonant frequencies at the ZGV points of the Lamb wave modes are more significant than those at the cut-off frequencies. This is consistent with previous observations on Lamb waves in homogeneous and composite plates. It was also shown that the material properties as well as the percentage of inversion layer have measurable effect on the occurrence of the first and second resonance peak frequencies. A comparison between the resonant frequencies obtained by this method and analytical approximations, usually adopted for transducer design, showed considerable differences, especially for the second resonant frequency. The modal analysis showed that the mechanical displacements and stresses are independent of the changes in the thickness of the inversion layer. The theoretical analysis presented in this study provides a useful means for accurately calculating high-order resonance modes and ZGV points. It is believed that the method presented here would be useful for the design and optimization of ultrasound transducers for harmonic imaging. 


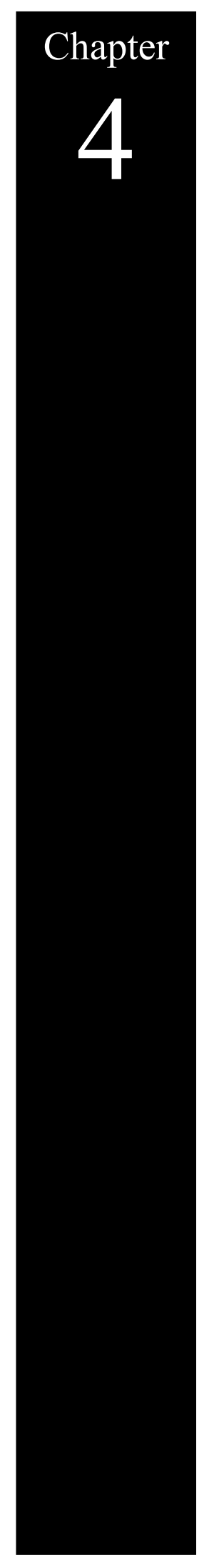

\section{Finite Width Plate Model for 1-D Array Transducers}

The content of the chapter is based on an article submitted to Ultrasonics

"Elastic guided wave propagation in a periodic array of multilayered piezoelectric plates with finite cross-sections"

Daniel H. Cortes

Subhendu K. Datta

Osama M. Mukdadi 


\subsection{Finite Element Formulation}

Multi-layered 1D-array ultrasound transducers are composed of a set of elements, each of them usually has one dimension much larger than the other two. In this study, each element is modeled as an infinite piezoelectric plate with a finite cross-section (Fig. 4.1). For the two geometries considered (Fig. 4.1), the cross section was discretized using 2D four-node finite elements in the $y z$-plane (azimuth plane). We assume harmonic wave motion in the $x$-axis (elevation direction). The displacement and electric potential are assumed to be time-dependent and functions of local coordinates $(x, y, z)$. These functions can be expressed in terms of the nodal values as follows (Mukdadi et al., 2002, 2003).

$$
\mathbf{v}(x, y, z, t)=\mathbf{N}^{e}(y, z) \mathbf{v}^{e}(x, t),
$$

where $\mathbf{v}$ is a $4 \mathrm{x} 1$ vector of the three components of displacement $(u, v, w)$ and the electric potential $(\phi)$ functions, $\mathbf{N}$ is a $4 \times 16$ matrix of interpolation functions and $\mathbf{v}^{e}$ is a $16 \times 1$ vector of nodal values. The strains and the electric field components can be expressed in a matrix form

$$
\mathbf{q}=\mathbf{L} \mathbf{v},
$$

where $\mathbf{q}=\left\{\mathrm{S}_{x x}, \mathrm{~S}_{y y}, \mathrm{~S}_{z z}, \mathrm{~S}_{y z}, \mathrm{~S}_{x z}, \mathrm{~S}_{x y}, \mathrm{E}_{x}, \mathrm{E}_{y}, \mathrm{E}_{z}\right\}^{T}$ and $\mathbf{L}^{*}$ is a $9 x 16$ matrix composed of spatial derivatives respect to the local coordinates. Note that $\mathbf{L}$ (Eq. 4.2) and $\mathbf{L}^{*}$ have different dimensions. The Hamilton's principle can be expressed as (see Appendix B for details):

$$
\delta \int_{t_{0}}^{t_{1}}\left[\frac{1}{2} \int\left(\dot{\mathbf{v}}^{e T} \mathbf{m}^{e} \dot{\mathbf{v}}^{e}-\mathbf{v}^{e T}{ }_{, x} \mathbf{k}_{11}^{e} \mathbf{v}_{, x}^{e}-\mathbf{v}_{, x}^{e T} \mathbf{k}_{12}^{e} \mathbf{v}^{e}-\mathbf{v}^{e T} \mathbf{k}_{21}^{e} \mathbf{v}_{, x}^{e}-\mathbf{v}^{e T} \mathbf{k}_{22}^{e} \mathbf{v}^{e}-\mathbf{v}^{e T} \mathbf{f}^{e}\right) d x\right] d t=0
$$

where $\mathbf{k}_{11}^{e}, \mathbf{k}_{12}^{e}, \mathbf{k}_{21}^{e}$ and $\mathbf{k}_{22}^{e}$ are element stiffness matrices, $\mathbf{m}^{e}$ is the element mass matrix and $\mathbf{f}^{e}$ is a vector of nodal forces. The global system of equations can be obtaining after assembling the element matrices and carrying out the variation of Eq. (4.3)

$$
\mathbf{K}_{1} \mathbf{V}_{, X X}-\mathbf{K}_{2} \mathbf{V}_{, X}-\mathbf{K}_{3} \mathbf{V}-\mathbf{M} \ddot{\mathbf{V}}=\mathbf{F} \text {. }
$$

Eq. (4.4) represents a system of linear partial differential equations. This system can be reduced to an algebraic system of equations applying Fourier transform as explained below.

\subsection{Frequency-domain Solution}

For a wave traveling in the $X$-direction, the Fourier transformation can be defined as

$$
\tilde{\mathbf{V}}(k, \omega)=\int_{-\infty}^{\infty} \int_{-\infty}^{\infty} \mathbf{V}(X, t) e^{-i(k X-\omega t)} d X d t
$$


where $k$ and $\omega$ represent the wavenumbers along $X$ direction and the angular frequency, respectively. Applying this transformation to Eq. (4.4), the partial differential system of equation is transformed into:

$$
\left(k^{2} \mathbf{K}_{1}-i k \mathbf{K}_{2}+\mathbf{K}_{3}\right) \tilde{\mathbf{V}}-\omega^{2} \mathbf{M} \tilde{\mathbf{V}}+\tilde{\mathbf{F}}=\mathbf{0} .
$$

Two eigenvalue problems arise when solving Eq. (4.6) for the homogeneous case $(\tilde{\mathbf{F}}=\mathbf{0})$. First, if $k$ is defined and the equation system (Eq. 4.6) is solved for $\omega$, the frequency spectra of the propagating modes are found. Second, when $k$ serves as eigenvalue, the equation system can be reorganized to reduce the order $k$ as follows:

$$
\left[\begin{array}{cc}
0 & \mathbf{I} \\
\omega^{2} \mathbf{M}-\mathbf{K}_{3} & i \mathbf{K}_{2}
\end{array}\right]\left\{\begin{array}{c}
\tilde{\mathbf{V}} \\
k \tilde{\mathbf{V}}
\end{array}\right\}-k\left[\begin{array}{cc}
\mathbf{I} & 0 \\
0 & \mathbf{K}_{1}
\end{array}\right]\left\{\begin{array}{c}
\tilde{\mathbf{V}} \\
k \tilde{\mathbf{V}}
\end{array}\right\}=\mathbf{0} .
$$

In this work, Eq. (4.6) is preferred to calculate the dispersion curves and the group and phase velocity spectra, since the number of equations is lower than that of Eq. (4.7).
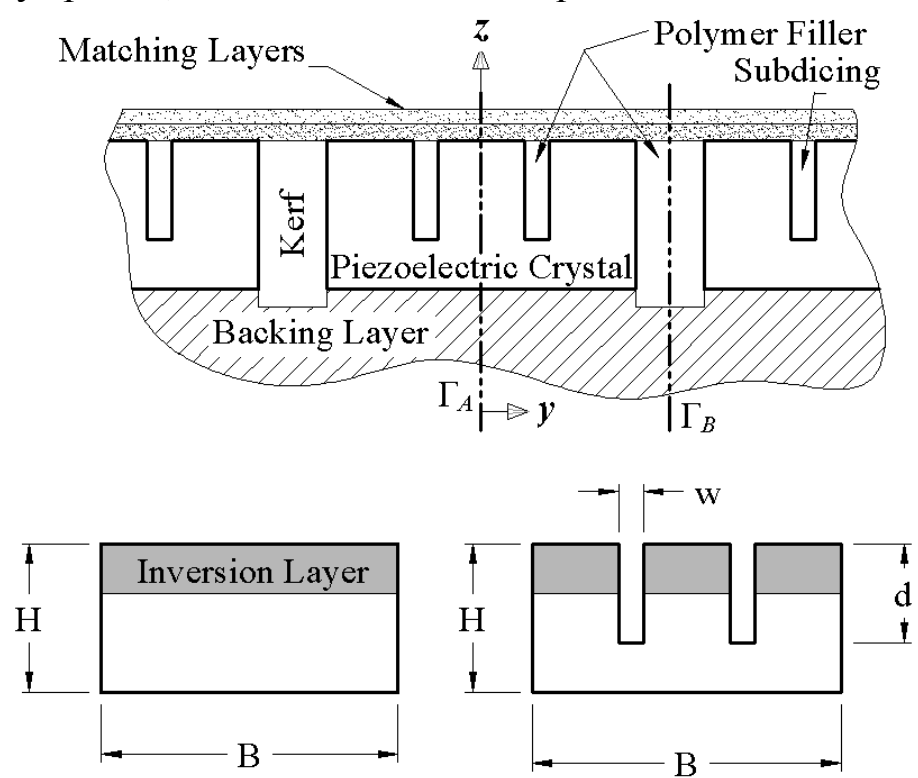

Figure 4.1. Rectangular and subdiced sections considered for this study, where $B$ is the piezoelectric element width, $H$ is the height, $w$ and $d$ are the subdicing width and depth, respectively.

\subsection{Boundary Conditions}

It can be observed from Figure 1 that the geometry of the transducers considered in this study has two symmetry axes $\left(\Gamma_{A}\right.$ and $\left.\Gamma_{B}\right)$. Notice that the electrical excitation is also symmetric about those axes. Therefore, symmetric boundary conditions at $\Gamma_{A}$ and $\Gamma_{B}$ can be considered to analyze the dispersion behavior of these periodic structures (Caronti et al. 2005; Goldberg et al. 1997; Ballandras et al. 2005). As explained by Ballandras et al. (2005), symmetrical boundary conditions can be obtained by setting the displacement in the $y$-direction equal to zero

$$
v_{\Gamma_{A}}=v_{\Gamma_{B}}=0 .
$$


Additionally, Ballandras et al. (2005) showed the equivalence between symmetrical and periodic boundary conditions for an infinite array excited synchronously. On the other hand, traction-free condition is adopted for the upper and lower boundaries (Fig. 4.2). These conditions allow the analysis of the complete array with less computational effort. Although half cell is used in the calculations, the mode shapes will be presented using the complete cell for better understanding of the results.

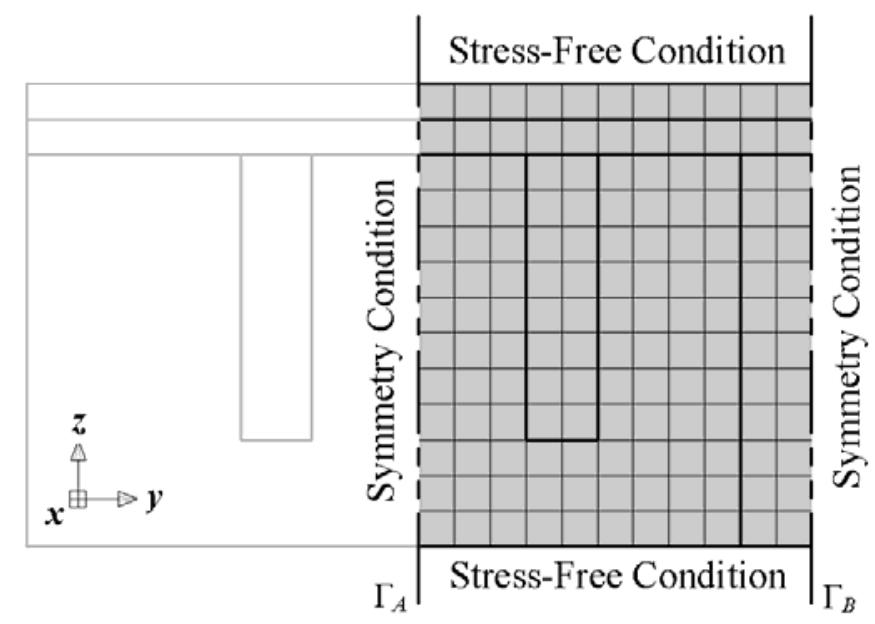

Figure 4.2. Schematic representation of FEM mesh and boundary conditions

\subsection{Parametric Analysis}

In this study, rectangular and subdiced cross sections for the 1-D array element are considered (Fig. 4.1). The piezoelectric material for all cases was $36^{\circ}$ rotated $\mathrm{Y}$-cut $\mathrm{LiNbO}_{3}$. A polymer filler is considered for kerfs and subdicing slots. The mechanical and piezoelectric properties for these materials are shown in Table 4.1. Although the formulation presented in this study is valid for any piezoelectric material, $36^{\circ}$ rotated $\mathrm{Y}$-cut $\mathrm{LiNbO}_{3}$ was chosen since it has been used in several studies for the design and fabrication of high frequency transducers for harmonic imaging (Nakamura et al. 2005; Nakamura et al. 2003; Zhou et al. 2005). The inversion layer can be generated in this material by a thermal process (Nakamura et al. 1992); therefore, there is not need of additional connections, electrodes or bonding between layers.

In this parametric study, we analyzed the effect of 1) the aspect ratio $B / H$ of the piezoelectric material, 2) the subdicing depth $d$ and width $w, 3$ ) the inclusion of $50 \%$ inversion layer, and 4) the inclusion of matching layers on the dispersion behavior and the thickness and lateral resonance frequencies of $1 \mathrm{D}$-array ultrasound transducers. The kerf width was set as $25 \%$ of the element width $B$ for all the models considered in this study. The matching layers were designed according the KLM model. The impedances of the first and second matching layers were selected as $z_{1}=z_{c}^{4 / 7} z_{l}^{3 / 7}$ and $z_{2}=z_{c}^{1 / 7} z_{l}^{6 / 7}$ (McKeighen, 1998), respectively. Where $z_{c}$ and $z_{l}$ are the acoustical impedances of the piezoelectric crystal and the load. The thickness was selected as a quarter of the dilatational wave length. Without loss of generality, the following non-dimensional parameters are used: $\Omega=\omega H / C_{s}$ is the non-dimensional angular frequency; $K=k H$ the 
non-dimensional wavenumber; $C_{g}=c_{g} / C_{s}$ the non-dimensional group velocity, and $C_{s}=\sqrt{C_{44} / \rho}$ the shear wave velocity. Tables 4.2 summarizes the geometric parameters considered in this article.

Table 4.1. Mechanical properties for polymer filler and matching layers.

\begin{tabular}{l|cccc}
\hline \multirow{2}{*}{ Material } & \multicolumn{3}{|c}{ Elastic Properties } & Density \\
& $C_{11}$ & $C_{12}$ & $C_{44}$ & $\left(\mathrm{Kg} / \mathrm{m}^{3}\right)$ \\
\hline Poly. Filler & 7.5 & 5.9 & 0.78 & 1080 \\
Matching Layer 1 & 24.5 & 3.35 & 5.3 & 3131.5 \\
Matching Layer 2 & 6.53 & 3.73 & 1.4 & 833 \\
\hline
\end{tabular}

\subsection{Results and Discussion}

Equations (4.6) and (4.7) represent the dispersion and resonance behavior of elastic guided wave propagation in piezoelectric plates with arbitrary cross-section. A custom Matlab (The Mathworks Inc., Natick, MA, USA) computer program was written to solve the dispersion relation (Eqs. 4.6 and 4.7) using appropriate periodic boundary condition (Eq. 4.8). Again, the periodic boundary conditions represent the symmetry of the piezoelectrical force excitation, geometry symmetry and periodicity of the considered structure. The number of elements in the horizontal and vertical directions was chosen for each aspect ratio to have elements with a height as close as possible to their width. Each model used approximately a total of 400 elements. Figure 4.3 shows the dispersion curves and group velocity spectrum of a periodic rectangular cross-section with a piezoelectric element aspect ratio $B / H(=2)$. Using the periodic boundary condition (Eq. 4.8) we obtained two types of waveguide modes: extensional $\mathrm{L}$ and flexural $\mathrm{B}_{\mathrm{Y}}$ (about $\mathrm{Y}$ axis). Figure 4.4 shows the dispersion curves and group velocity spectrum of a periodic rectangular cross-section with a piezoelectric element aspect ratio $B / H(=1.5)$. Comparison between Figs. 4.3 and 4.4 shows the effect of the piezoelectric element aspect ratio on the cut-off frequencies and thus on the resonance modes. Furthermore, close view windows show strong coupling between extensional and flexural modes. The mode coupling shown in Figs 4.3 and 4.4 is an evident that the guided wave modes are quasi extensional and flexural modes (Mukdadi et al., 2003, 2002).

Table 4.2. Geometric parameters for all the models considered.

\begin{tabular}{|c|c|c|c|c|c|c|}
\hline Cross Section & Figure & Sketch & $\%$ of IL & $B / H$ & $d / H$ & $\mathbf{w} / \mathbf{B}$ \\
\hline \multirow{3}{*}{ Rectangular } & 4.3 & & 0 & 2 & -- & -- \\
\hline & 4.4 & & 0 & 1.5 & -- & -- \\
\hline & 4.5 & & 50 & 2 & -- & -- \\
\hline
\end{tabular}




\begin{tabular}{|c|c|c|c|c|c|}
\hline \multirow{6}{*}{ Subdiced } & 4.6 & 0 & 2 & 0.9 & 0.2 \\
\hline & 4.7 & 0 & 1.5 & 0.9 & 0.2 \\
\hline & 4.8 & 0 & 2 & 0.7 & 0.2 \\
\hline & 4.9 & 0 & 2 & 0.9 & 0.15 \\
\hline & 4.10 & 50 & 2 & 0.9 & 0.2 \\
\hline & 4.11 & 0 & 2 & 0.9 & 0.2 \\
\hline
\end{tabular}

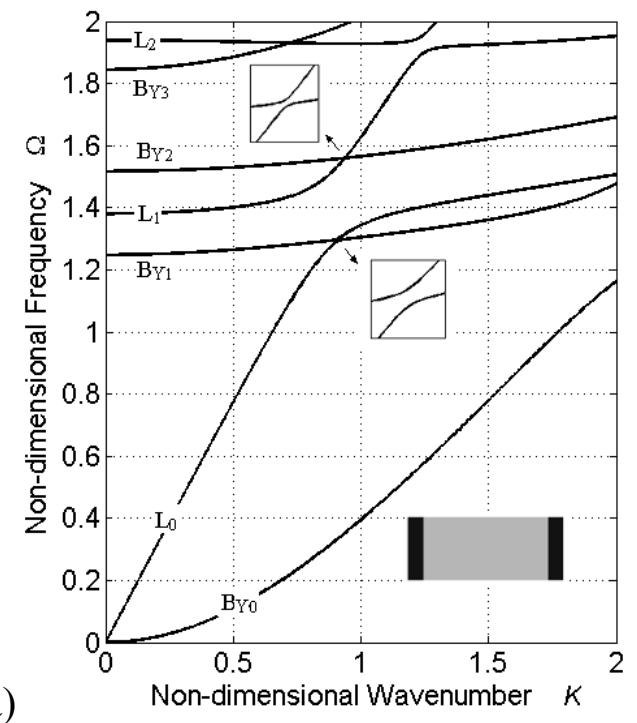

b)

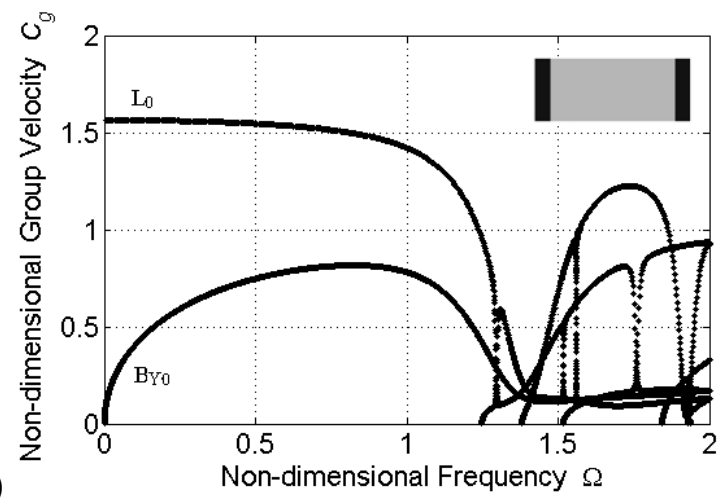

Figure 4.3. Dispersion curves and group velocity spectra for a rectangular cross-section with $B / H=2$ and periodic boundary condition. 


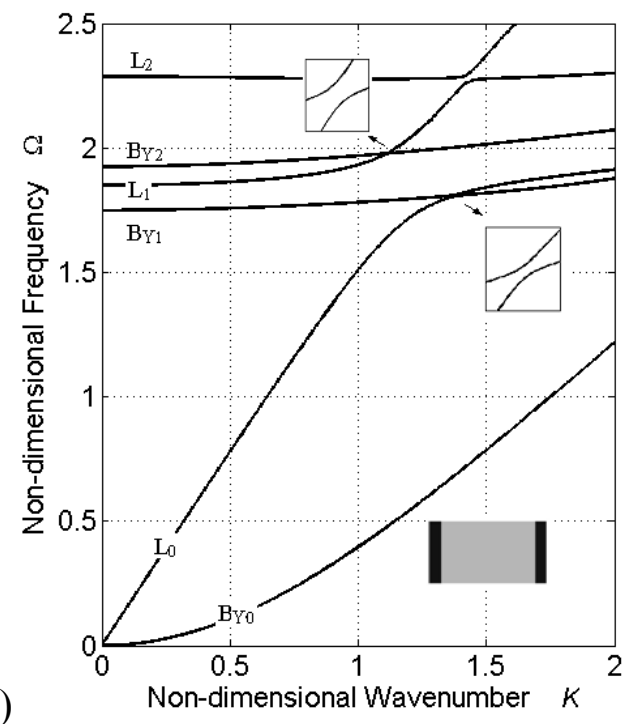

b)

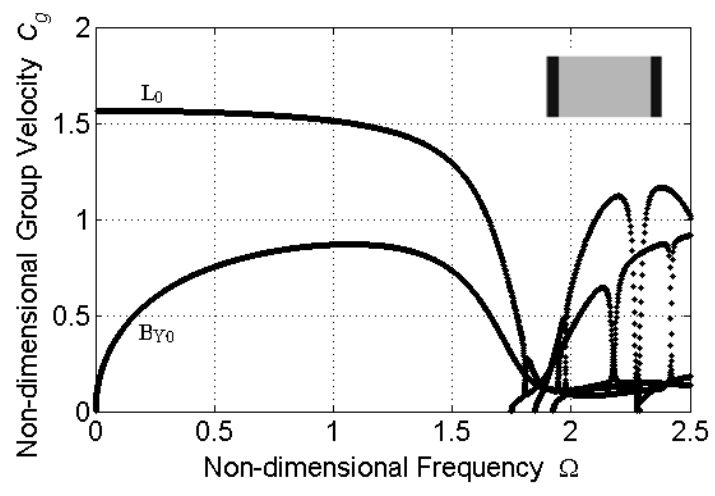

Figure 4.4. Dispersion curves and group velocity spectra for a rectangular cross-section with $B / H=1.5$ and periodic boundary condition.

Figure 4.5 shows the dispersion behavior of guided wave propagation in layered piezoelectric plate element with rectangular cross-section and inversion layer. The layered structure consists of an inversion layer on top of a $36^{\circ} \mathrm{Y}$-cut $\mathrm{LiNbO}_{3}$ layer. The inverted layer has similar elastic properties that are used for $36^{\circ} \mathrm{Y}$-cut $\mathrm{LiNbO}_{3}$ but with inverted dielectric constants (Zhou et al., 2005). The thickness ratio used for the inverted layer is $50 \%$ of the total thickness of the piezoelectric plate. In this figure, mode coupling is clearly shown between $L_{0}$ and $B_{Y 1}$ and between $L_{1}$ and $B_{Y 2}$. A close view is illustrating the mode interchange. Comparison between Figs. 4.3 and 4.5 indicates that the inversion layer appears to lower both the cut-off frequencies and the group velocities. Another feature appears in Figs. 4.3, 4.4 and 4.5 for piezoelectric plates with rectangular cross-sections is the absence of the zero-group-velocity points within the region of interest shown in the figures.

To illustrate the effect of the subdicing micro-fabrication process used in industry theoretical dispersion and group velocity analysis is shown in Fig. 4.6 for a piezoelectric plate with aspect ratio $B / H(=2)$, subdicing depth $d / H(=0.9)$, and subdicing width $w / B(=$ 0.2 ). A periodic boundary condition is applied at the edges of the polymer filling as shown in Fig. 4.6. Comparison between rectangular and subdiced cross-sections (see, Figs. 4.3 and 4.6) shows a drastic change of guided wave dispersion and resonance behavior. Guided wave propagation in subdiced piezoelectric plate (Fig. 4.6) exhibits the following interesting features; 1) increase in the number of the lateral modes; 2) lower cut-off frequencies; 3) strong mode coupling between any two successive modes, which indicate quasi-mode behavior; 4) increase in the number of zero-group-velocity points; 5) lower flexural and surface wave velocity; and 6) lower extensional $\left(\mathrm{L}_{0}\right)$ group velocity at zero wavenumber (i.e., $k=0$ ). Some of these features are strongly influence the resonance behavior of piezoelectric plate and thus alter the transducer resonance frequencies and the overall bandwidth. 
Figure 4.7 shows the effect of the piezoelectric aspect ratio $B / H$ on the dispersion behavior. Comparison between Figs. 4.6 and 4.7 indicates that increasing the aspect ratio will increase the number of resonance modes at cut-off frequencies. Also, comparing Figs. 4.4 and 4.7 shows the effect of the subdicing process on the resonance characteristics. Analogous features of the subdicing effects mentioned above are also observed in this figure. This theoretical study provides a means to clearly elucidate the effect of piezoelectric material and geometry on the dispersion behavior, characteristic resonance frequencies, and group velocity spectrum.

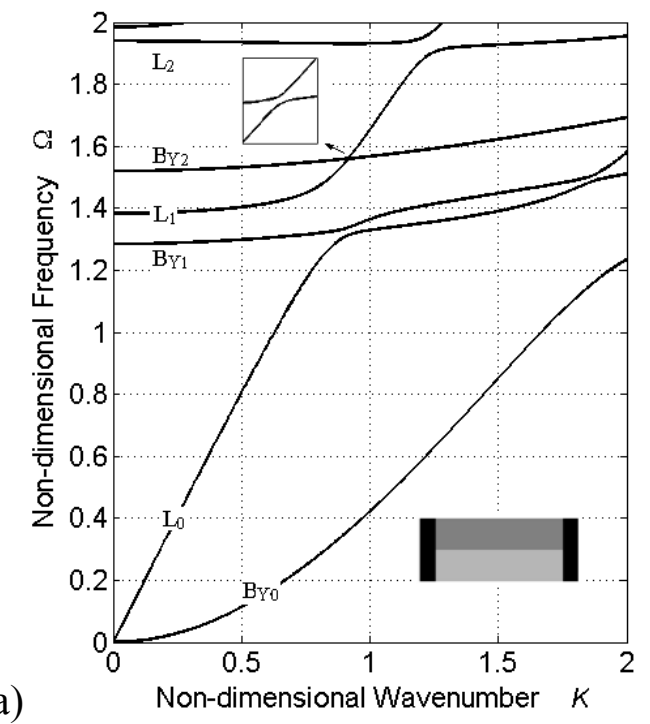

b)

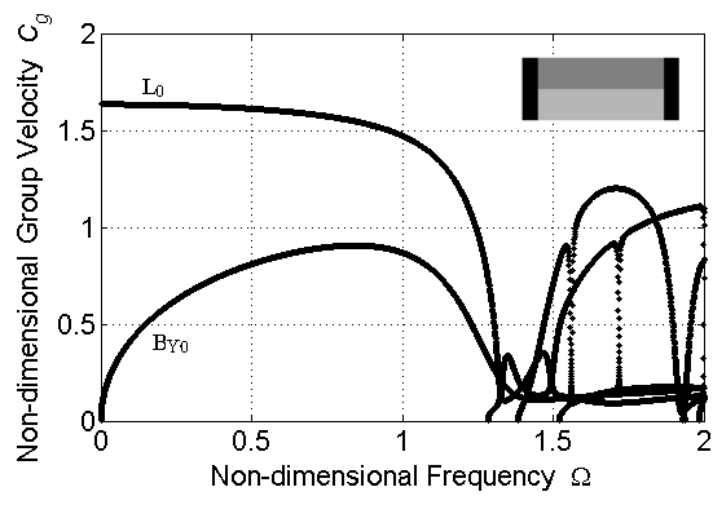

Figure 4.5. Dispersion curves and group velocity spectra for a rectangular cross-section with $B / H=2$, inversion layer and periodic boundary condition.

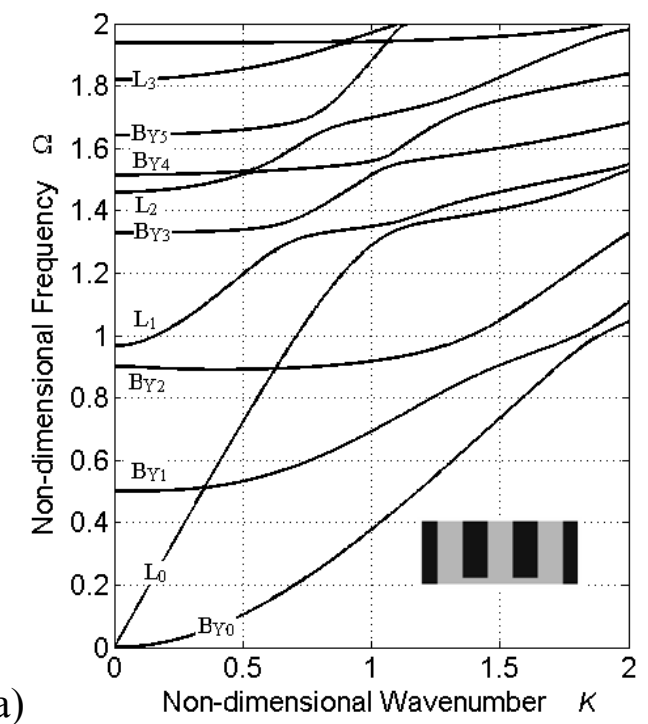

b)

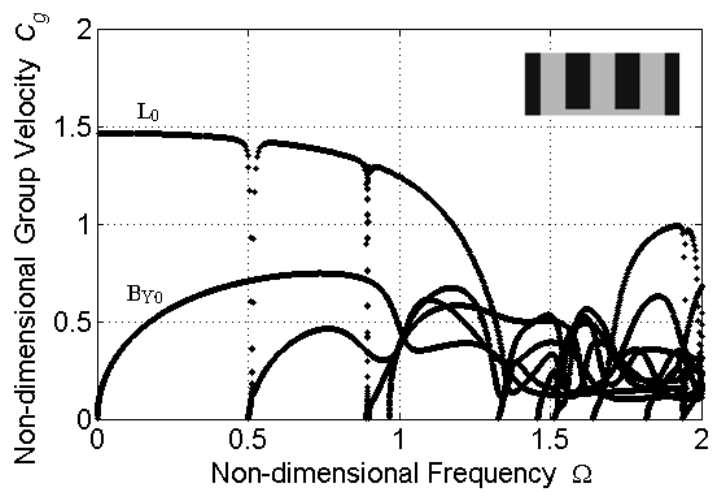

Figure 4.6. Dispersion curves and group velocity spectra for the subdiced section with $B / H=2, d / H=0.9, w / B=0.2$ and periodic boundary condition. 

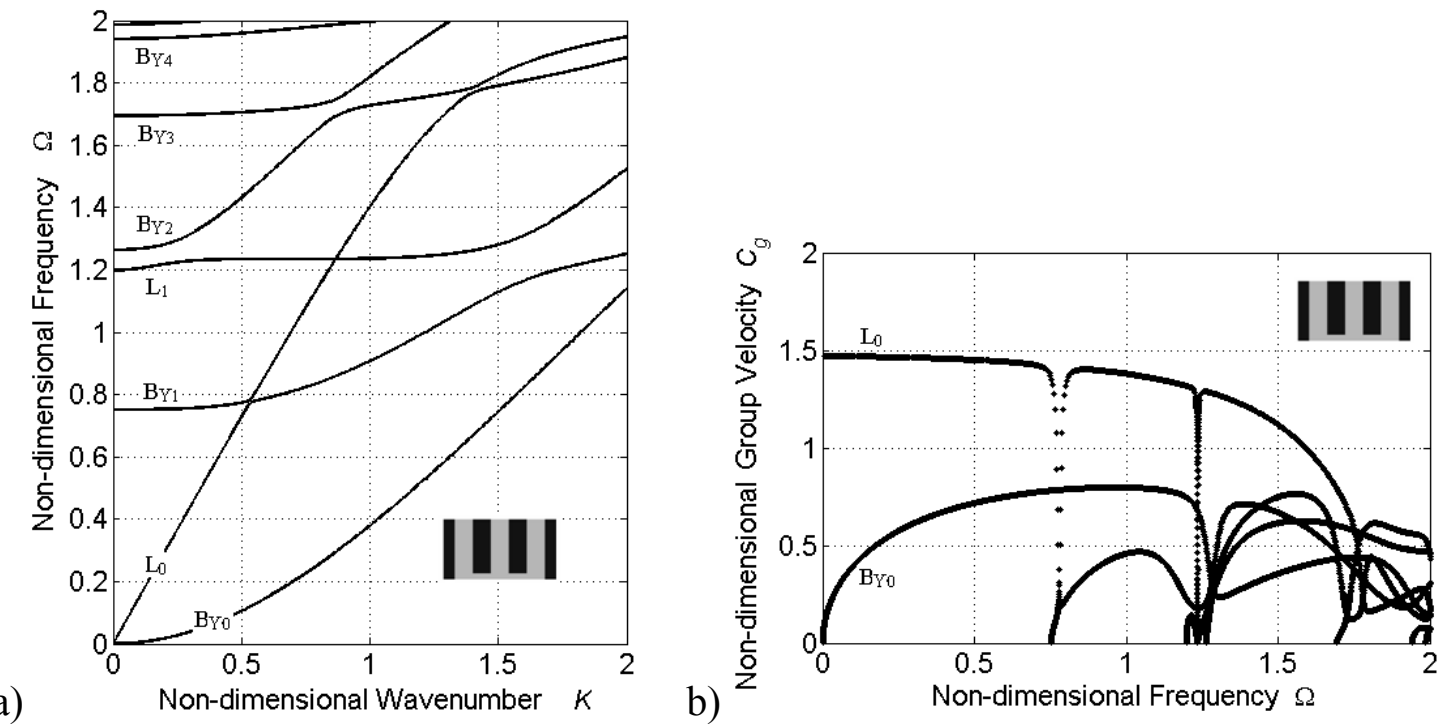

Figure 4.7. Dispersion curves and group velocity spectra for the subdiced section with $B / H=1.5, d / H=0.9, w / B=0.2$ and periodic boundary condition.
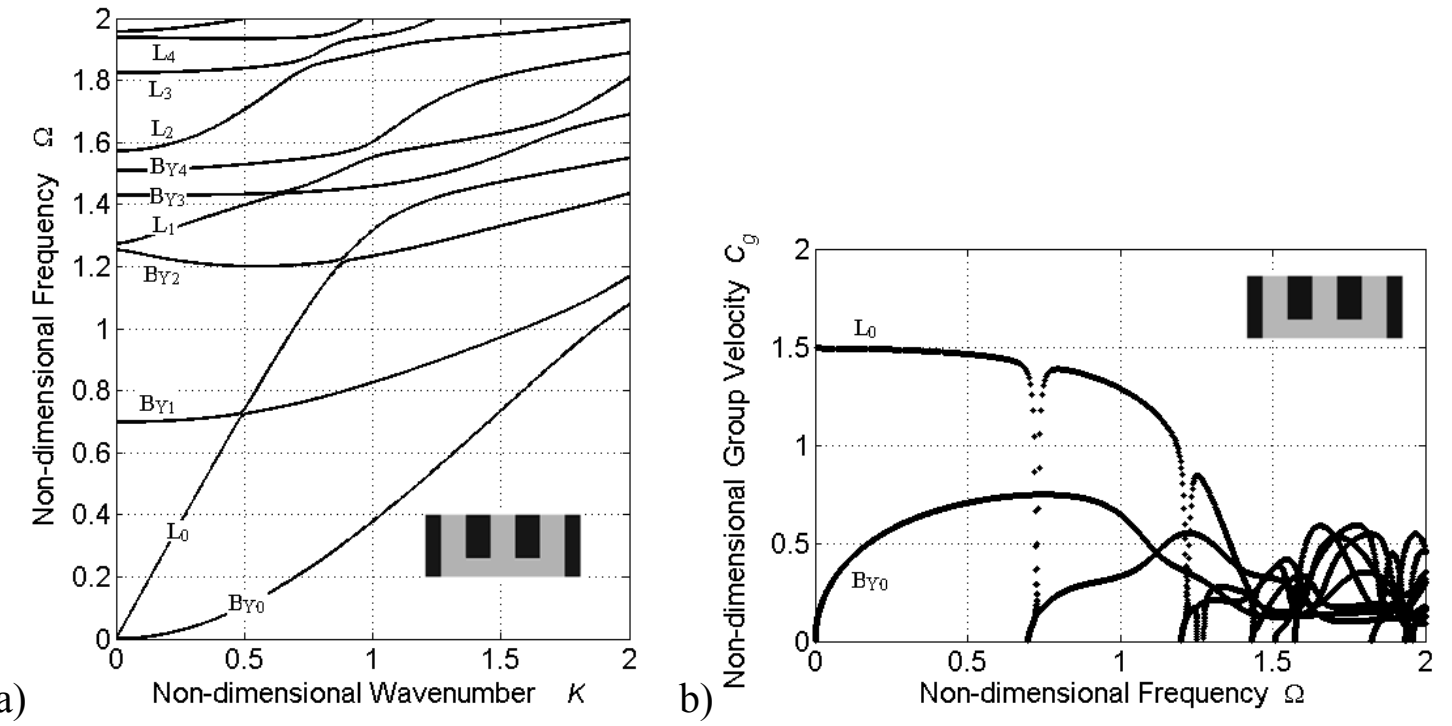

Figure 4.8. Dispersion curves and group velocity spectra for the subdiced section with $B / H=2, d / H=0.7, w / B=0.2$ and periodic boundary condition.

The piezoelectric subdicing process is mainly limited by both the thickness of the dicing saw and the material and thickness of the piezoelectric material. These factors determine the ratios of the element subdicing width $(w / B)$ and depth $(d / H)$. To study the effect of these two parameters, we calculated the dispersion curves and the group velocity spectrum in Figs. 4.9 and 4.10, respectively. Figure 4.9 shows the dispersion behavior of guided wave propagation in a subdiced piezoelectric plate with aspect ratio $B / H(=2)$, subdicing width $w / B(=0.2)$, and subdicing depth $d / H(=0.7)$. Note that in this case, the piezoelectric element has an analogous geometry to that presented in Fig. 4.7 but lower 
subdicing depth $d / H(=0.7)$. The effect of subdicing depth $(d / H)$ is clearly shown to cluster high-order waveguide modes between $\mathrm{L}_{1}$ and $\mathrm{L}_{3}$ extensional modes. Also, fewer modes are observed in the same frequency range in Fig. 4.9. This effect may be due by the increase of the rigidity caused by the small depth of the subdicing. Furthermore, the first extensional mode $\mathrm{L}_{1}$ exhibits zero-group-velocity point, where in Fig. 4.7 the second flexural $\mathrm{B}_{\mathrm{Y} 2}$ mode shows similar ZGV point. These ZGV points have strong resonance characteristics that could be used for designing 1D-array ultrasound transducers. Figure 4.10 illustrates the effect of the subdicing width $w / B$ of the piezoelectric plate on the dispersion behavior. Comparison between Fig. 4.7 and Fig. 4.10 shows a slight difference in the dispersion behavior and group velocity spectrum. This may imply that the effect of subdicing width is not as important as that of the depth.
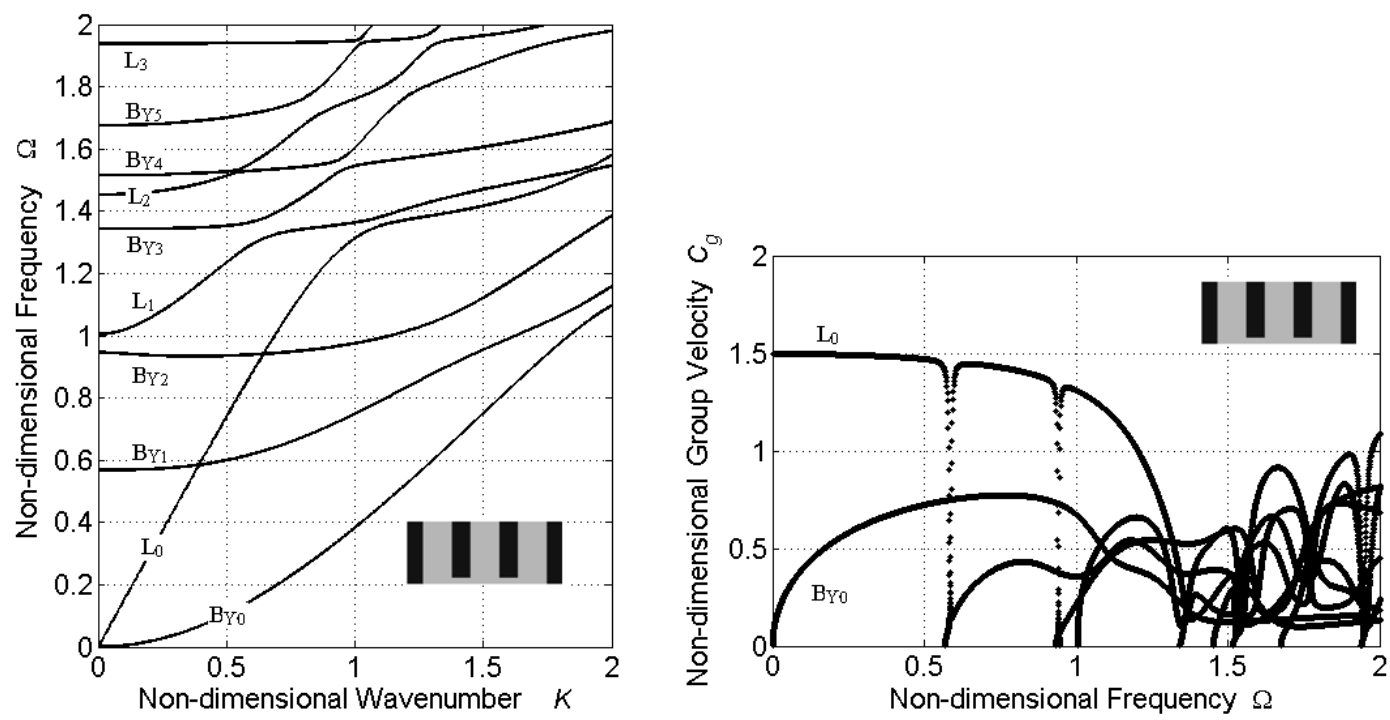

Figure 4.9. Dispersion curves and group velocity spectra for the subdiced section with $B / H=2, d / H=0.9, w / B=0.15$ and periodic boundary condition.

The dispersion curves and group velocity spectra for the subdiced cross-section with an inversion layer are shown in Fig. 4.11. Comparison between the results for the subdiced cross-section (Fig. 4.11) and Fig. 4.7 shows small differences for all the modes. In general, inversion layer has a minor effect on the dispersion behavior of guided wave propagation. This could be explained by the significant influence of the geometric factors of the subdiced piezoelectric element rather than the material selection on generating both lateral and thickness guided waves.

Matching layers have an important effect on the dispersion curves and their corresponding group velocity spectra (Fig. 4.12). In this case, the dispersion curves show that extra modes appear especially at high frequencies. This may be due to the additional restriction to the lateral movement of the piezoelectric columns imposed by the matching layers. The effect of the matching layers on the dynamic response of ultrasound transducer was addressed by Zhou et al. ${ }^{16}$ who found the frequencies with optimum efficiency changed when different configuration of matching layer were considered. 


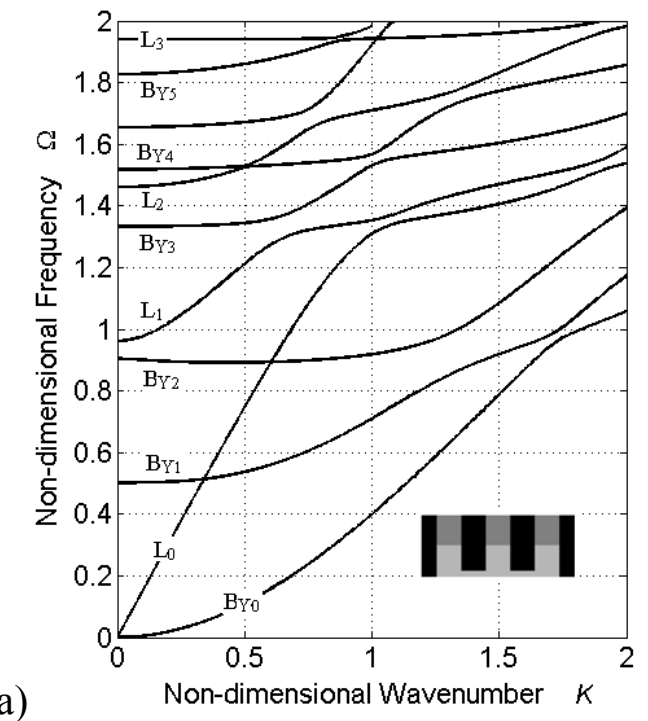

b)

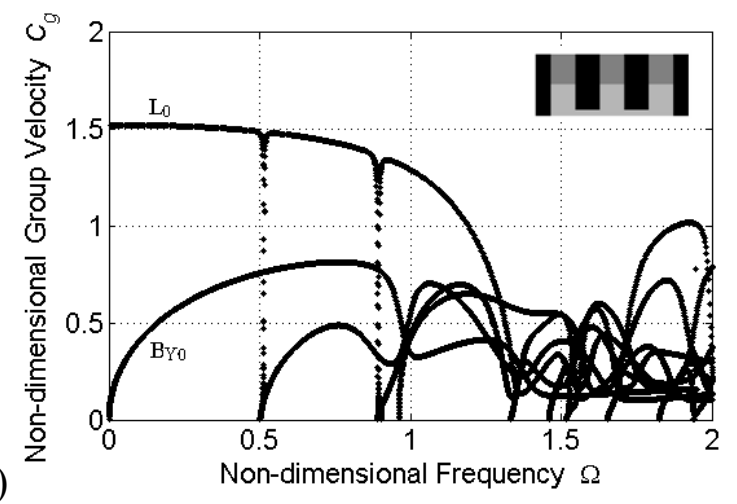

Figure 4.10. Dispersion curves and group velocity spectra for the subdiced section with $B / H=2, d / H=0.9, w / B=0.2$, inversion layer and periodic boundary condition.

Both thickness and lateral-mode resonance frequencies can occur at cut-off frequencies (i.e., wavenumber $k=0$ ) and at zero-group-velocity (ZGV) points (i.e., wave group velocity $C_{g}=0$ ). Mode shapes at the cut off frequency for the first and second order modes are shown in Fig. 4.13. A close look into the deformed shapes reveals that some of the extra-modes present in the subdiced section are associated with the lateral movement of the piezoelectric columns. The mode shapes can be divided into extensional and flexural groups. Extensional modes are characterized by quasi symmetric displacements about $\mathrm{Y}$-axis. In contrary, flexural modes indicate quasi antisymmetric wave displacements about Y-axis. It is clearly shown that there are two types of resonance behavior of these extensional and flexural modes. First, there are resonance modes with significant in-plane displacements (YZ plane) with negligible out-of the plane displacement along $\mathrm{X}$-axis. These modes could be quasi symmetric extensional (L) modes or quasi anti-symmetric flexural modes as shown in Fig. 4.13. These modes with dominant in-plane thickness and lateral displacements of the piezoelectric pillars may increase the acoustic emission and the frequency bandwidth of the transducer. Second, mode shapes with significant out-of the plane displacement along X-axis but negligible in-plane displacements (in YZ plane). The latter modes will not play a significant role in improving the acoustic emission not the frequency bandwidth of the transducer. 

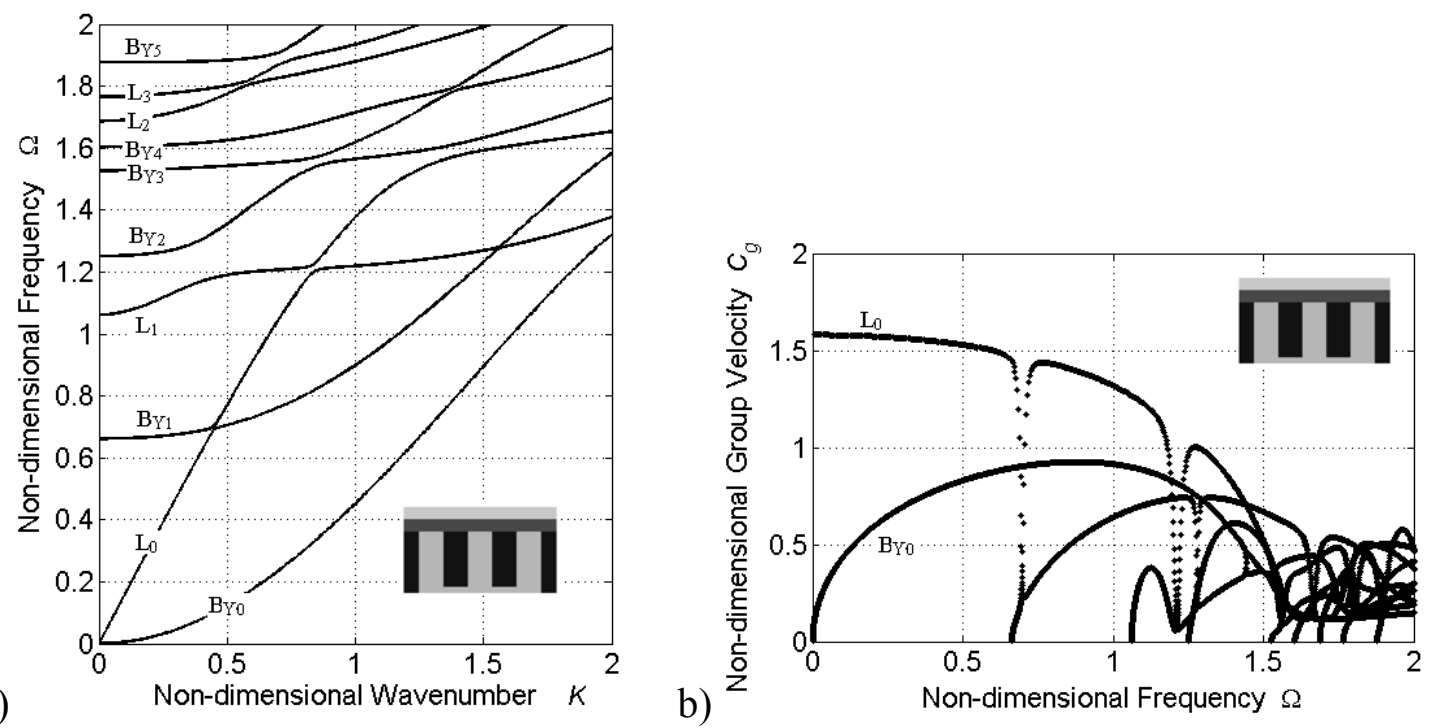

Figure 4.11. Dispersion curves and group velocity spectra for the subdiced section with $B / H=2, d / H=0.9, w / B=0.2$ two matching layers and periodic boundary condition. 


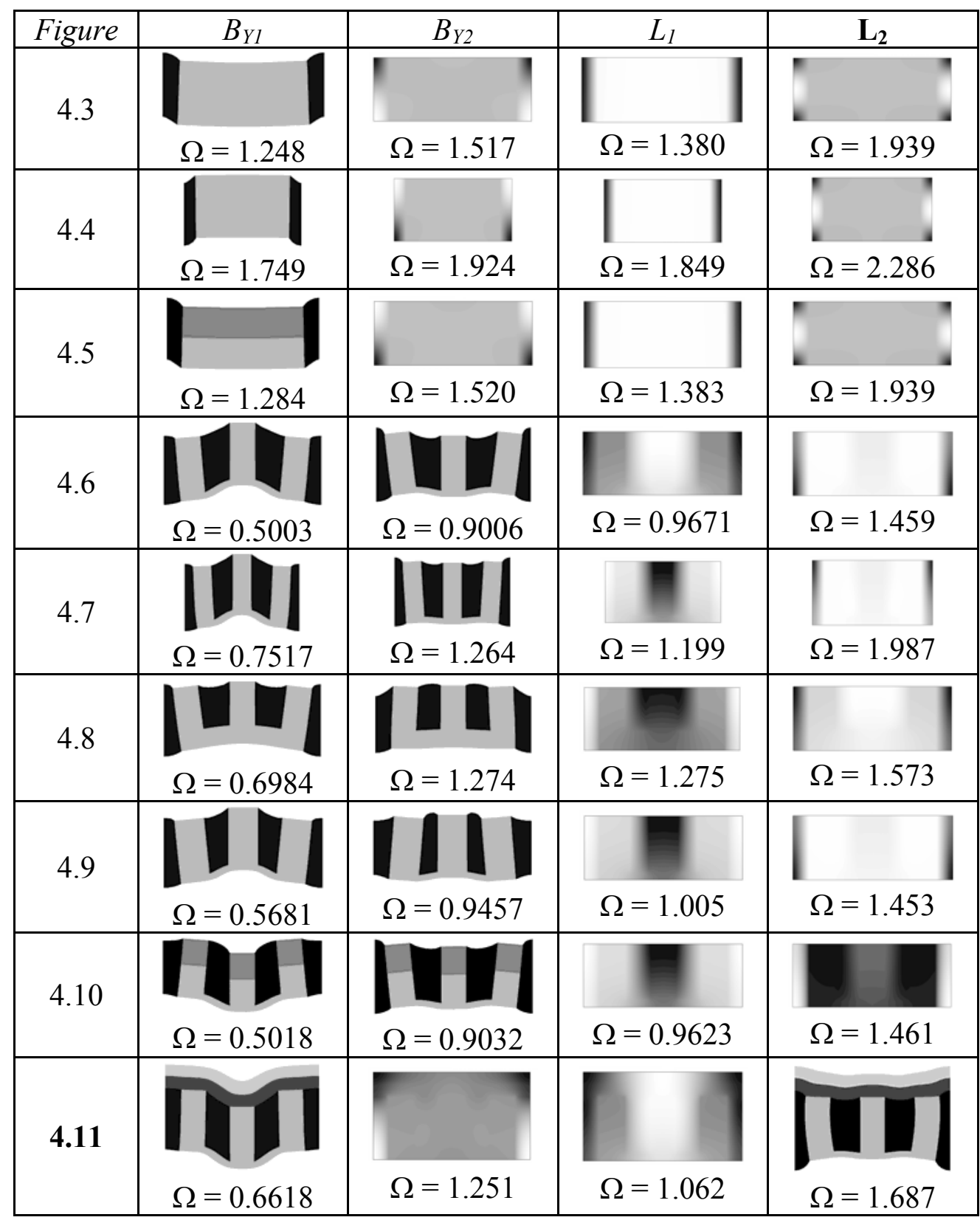

Figure 4.12. Mode shapes at cut-off frequencies (wavenumber $k=0$ ) of the first two extensional and flexural modes about Y-axis for all the geometries considered. Extensional modes are characterized by the quasi symmetry of displacements about $Y$ axis. Conversely, flexural modes have quasi anti-symmetric displacements about the $Y$ axis. Density plots shown here correspond to displacements along the $X$-axis, $Y Z$ plane displacements for those modes are negligible. 


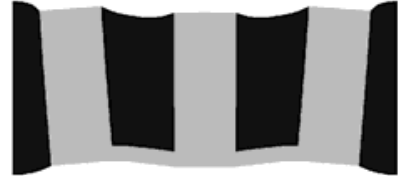

$K=0.412, \Omega=0.8905$

(a)

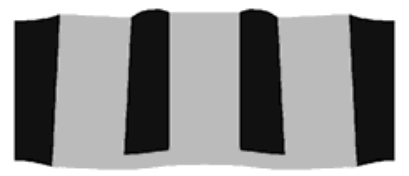

$K=0.360, \Omega=0.9335$

(c)

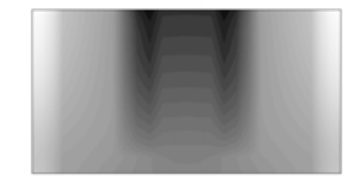

$K=0.547, \Omega=1.200$

(b)

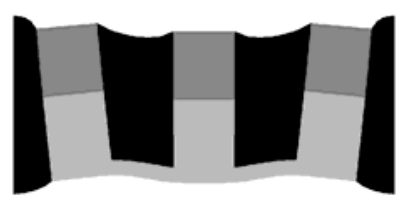

$K=0.452, \Omega=0.8913$

(d)

Figure 4.13. Mode shapes corresponding to the zero group velocity points in (a) Fig. 4.7, $\mathrm{B}_{\mathrm{Y} 2}$ mode, (b) Fig. 4.8, $\mathrm{L}_{1}$ mode, (c) Fig. 4.10, $\mathrm{B}_{\mathrm{Y} 2}$ mode, (d) Fig. 4.11, $\mathrm{B}_{\mathrm{Y} 2}$ mode. Values of the wavenumber $k$ and angular frequency $\omega$ are also shown for each ZGV point. For subfigure (b), the $Y Z$-plane displacements are negligible compared with the $X$ axis displacements (shown as a density plot); therefore, they are not shown.

Figure 4.13 shows plots of the mode shapes at zero-group-velocity points. These mode shapes exhibit a resonance behavior analogous to that shown at cut-off frequencies. However, the associated wavenumber is not equal to zero (i.e. $k \neq 0$ ) as shown in Fig. 4.13. $\mathrm{ZGV}$ points below the cut-off frequency in the second-order flexural $\mathrm{B}_{\mathrm{Y} 2}$ mode were found in all subdiced geometries. In addition, a ZGV above the cut-off frequency for the first-order extensional $\mathrm{L}_{1}$ mode was observed in Fig. 4.8. The mode shapes for ZGV points on Figs. 4.7, 4.8, 4.9 and 4.11 are shown in Fig. 4.13. It can be clearly seen the similarity between these modal shapes and those of the cut-off frequencies of the corresponding mode. Again, analogous behavior to that indicated in Fig. 4.12 can be deduced. Here, resonance modes with significant in-plane (YZ plane) displacements are of interest for designing multi-resonance ultrasound transducers. Figure 4.13 (a, c and d) show strong in-plane displacements at $\mathrm{ZGV}$ points of the second-order flexural $\mathrm{B}_{\mathrm{Y} 2}$ mode. On the other hand Fig. 4.13(b) indicates significant out of the plane displacement (along X-axis), yet weak in-plane displacement of the first-order extensional $\mathrm{L}_{1}$ mode. This analysis provides a comprehensive assessment of the resonance modes in periodic piezoelectric plates for 1D ultrasound transducers, and whether these resonance modes will be significant for multi-resonance ultrasound transducers.

\subsection{Concluding Remarks}

In this work, 1D-array ultrasound transducers are modeled as periodic piezoelectric plates with finite cross-section. A semi-analytic finite-element formulation is adopted to analyze the dispersion behavior of these structures. Dispersion curves, group velocity spectra and mode shapes are obtained using the method presented here. 
Geometric parameters such as aspect ratio and subdicing width and depth had an important effect on the dispersion behavior. In general, the lower aspect ratios, as well as lower subdicing depth, tend to increase the cut-off frequency of all modes. Inversion layer was shown to have discrete effect of the dispersion relation for this type of structures. Matching layers caused some resonance modes to cluster over a certain frequency range. It was also shown that the mode shapes for the zero-group-velocity points show characteristic resonance behavior analogous to that at the cut-off frequencies. This study shows that both material anisotropy and subdiced cross-section play a strong role on the mode coupling behavior and zero-group-velocity of guided waves. In-plane and out of the plane wave displacements can be easily evaluated for ultrasound transducer design. These factors generate more complicated dispersion and resonance characteristics of 1D-array ultrasound transducers. The method presented here can be used to analyze complicated geometries to get useful information for design of such transducers and a better understanding of the dynamic response of 1D-array ultrasound transducers. 


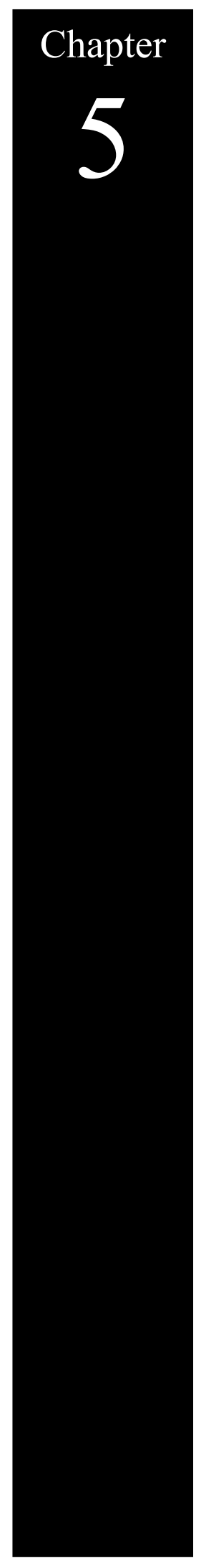

\section{Analytical Modeling of Single-Element Ultrasound Transducer using Leaky Lamb Waves}

The content of the chapter is based on an article submitted to IEEE Transactions on Ultrasonics, Ferroelectrics, and Frequency Control

"Characterization of single-element transducers using leaky Lamb wave analysis"

Daniel H. Cortes

Subhendu K. Datta

Osama M. Mukdadi 


\subsection{Introduction}

The design of ultrasound transducer is commonly based on equivalent circuits such as KLM, Mason, and Redwood. In these models, the dynamic characteristics of the piezoelectric element, such as resonance frequency, are usually obtained using equivalent models, which may not represent accurately the behavior of these devices. In this study, the mechanical behavior of single element transducer is modeled analytically. In addition, the effect of the fluid loading and matching and backing layer on the dispersion curves, resonance frequencies and other dynamic parameters is analyzed. An analytical model of leaky Lamb wave propagation in a thin piezoelectric plate surrounded a solid half-space on bottom and another half-space of tissue-like material on top is implemented to calculate the dispersion curves, resonance and attenuation behavior. Several important changes on the dynamic characteristics of the transducer are observed when the piezoelectric plate is bonded to the backing layer. The plate bonded to a backing layer has multiple resonance frequencies corresponding to $\mathrm{A}_{1}, \mathrm{~S}_{1}, \mathrm{~A}_{2}$, and $\mathrm{S}_{4}$ wave modes. These modes may be associated with the mutual interaction between the plate and the backing layer. On the other hand, only $\mathrm{S}_{1}$ and $\mathrm{S}_{4}$ modes are observed when the backing layer is removed. Interestingly, these resonance frequencies do not correspond to the cutoff frequencies or to the zero-group-velocity points as known in the case of Lamb wave propagation in plates surrounded by air or vacuum. This study shows that adding a backing layer affects the resonance characteristics, in which the resonance will occur at transition frequencies where the magnitude of the wavenumber reaches a minimum value $(\min |\mathrm{K}|)$. The addition of matching layers increases the number of modes present in the frequency range considered. This study may be helpful to predict the dynamic behavior of piezoelectric devices, such as, single-element ultrasound transducers and Lamb wave acoustic sensors.

\subsection{Analytical Model}

\subsubsection{Model Description}

In this section, the dynamic characteristics of laminated piezoelectric structures, such as ultrasound transducers, are theoretically analyzed. Usually, the transducer is composed of elastic and piezoelectric layers on top of a thick layer called backing layer (Figure 5.1). To model the transducer, a multilayered plate surrounded by a solid halfspace, which represents the backing layer, is considered. Another half-space of 'tissuelike' fluid, representing the tissue to be imaged, needs to be considered at the front side of the transducer. In this study, we are modeling the tissue using fluid acoustic properties. Figure 5.1 shows a schematic plot of single-element ultrasound transducer. It is expected that the presence of the backing layer and tissue around the piezoelectric plate significantly alters wave propagation characteristics, such as attenuation and wave velocities. The wave propagation can be modeled by means of leaky Lamb waves, where the surrounding media will be considered.

The theoretical analysis presented here is based on the partial wave approach, in which the constitutive relations and the motion equations are combined to find all the 
possible wave components (partial waves) for a given mode. The partial waves are function of the wavenumber and the frequency. However, these two parameters are not independent from each other. To satisfy the boundary conditions, it found the wavenumber and the frequency must be related. That relation is known as the dispersion equation. The dispersion equation provides important information such as phase and group velocities, cut-off frequencies and attenuation.

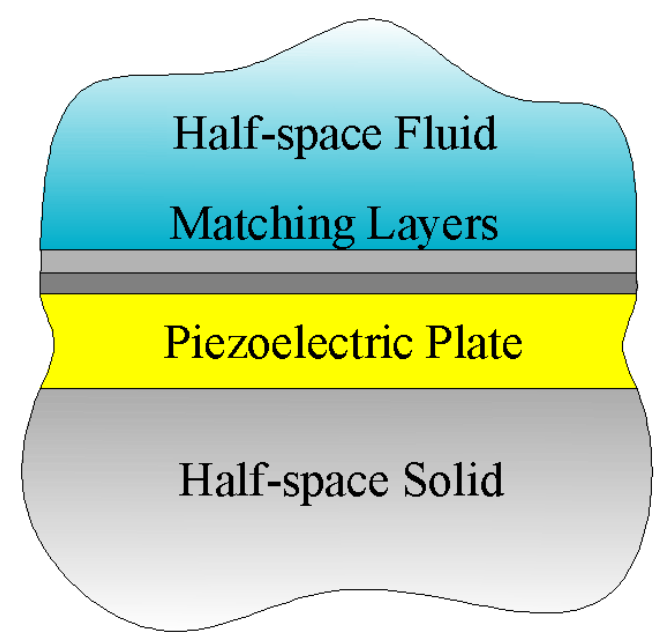

Figure 5.1. Schematic representation of the analytical model for analyzing the wave propagation on single-element ultrasound transducers.

\subsubsection{Dispersion Relations}

The dispersion equations are the relation between the frequency and the wavenumber of a particular wave mode. When the wavenumber is proportional to the frequency, the wave is called non-dispersive. However, leaky Lamb waves are dispersive; this indicates that the dispersion relations are function of the material properties, fluid properties and dimensions of the layered plate. In this section, an analytical procedure to obtain the dispersion behavior of leaky Lamb waves propagating in a piezoelectric layered plate and surrounded by a fluid and a solid half-spaces is presented.

In order to obtain the solution in the frequency domain, all the variables are Fourier transformed as follows

$$
\hat{\varphi}=\int_{-\infty}^{\infty} \int_{-\infty}^{\infty} \varphi e^{i \omega t} e^{-i \zeta x_{1}} d t d x_{1} .
$$

where $\zeta$ is the wavenumber in the $x_{1}$ direction and $\omega$ is angular frequency. The displacements, stresses and electrical displacement and potential can be stated, after transformation (Nayfeh, 1995), as

$$
\begin{gathered}
\left(\hat{u}_{1}^{m}, \hat{u}_{3}^{m}, \hat{\phi}^{m}\right)=\sum_{q=1}^{6}\left(U_{q}^{m}, W_{q}^{m}, \Phi_{q}^{m}\right) \beta_{q}^{m} e^{i \zeta \zeta_{q}^{m} x_{3}}, \\
\left(\hat{T}_{33}^{m}, \hat{T}_{13}^{m}, \hat{D}_{3}^{m}\right)=\sum_{q=1}^{6} i \zeta\left(D_{1 q}^{m}, D_{2 q}^{m}, D_{3 q}^{m}\right) \beta_{q}^{m} e^{i \zeta \alpha_{q}^{m} x_{3}},
\end{gathered}
$$


where $m$ indicates the layer considered, $q$ corresponds to the partial wave, $\alpha_{q}^{m}$ the ratio between the wavenumber in the $x_{3}$ and $x_{1}$ directions for the layer $m$ and the partial wave $q$, the coefficients $U_{q}^{m}, W_{q}^{m}$ and $\Phi_{q}^{m}$ are generalized coordinates for the displacements and the electric potential, $\beta_{q}^{m}$ is an unknown coefficient for the partial wave $q$ of the layer $m$, the coefficients $D_{i q}$ can be expressed in terms of the elastic stiffness matrix of the layer $m\left(C_{i j}^{m}\right), \alpha_{q}^{m}$ and $\zeta$ (Lee and Kuo, 2006).

$$
\begin{gathered}
D_{1 q}^{m}=C_{13}^{m} U_{q}^{m}+C_{35}^{m} W_{q}^{m}+C_{35}^{m} \alpha_{q}^{m} U_{q}^{m}+C_{33}^{m} \alpha_{q}^{m} W_{q}^{m}+e_{13}^{m} \Phi_{q}^{m}+e_{33}^{m} \alpha_{q}^{m} \Phi_{q}^{m} . \\
D_{2 q}^{m}=C_{51}^{m} U_{q}^{m}+C_{55}^{m} W_{q}^{m}+C_{55}^{m} \alpha_{q}^{m} U_{q}^{m}+C_{53}^{m} \alpha_{q}^{m} W_{q}^{m}+e_{15}^{m} \Phi_{q}^{m}+e_{35}^{m} \alpha_{q}^{m} \Phi_{q}^{m} . \\
D_{3 q}^{m}=e_{31}^{m} U_{q}^{m}+e_{35}^{m} W_{q}^{m}+e_{35}^{m} \alpha_{q}^{m} U_{q}^{m}+e_{33}^{m} \alpha_{q}^{m} W_{q}^{m}-\varepsilon_{13}^{m} \Phi_{q}^{m}-\varepsilon_{33}^{m} \alpha_{q}^{m} \Phi_{q}^{m} .
\end{gathered}
$$

The displacements and pressure for the fluid on top of the plate can be expressed as (Lee and Kuo, 2006)

$$
\left(\hat{u}_{1}^{f}, \hat{u}_{3}^{f}, \hat{T}_{33}^{f}\right)=\left(1,-\alpha_{f}, i \rho^{f} \omega^{2} / \zeta\right) \beta_{f} e^{i \zeta \alpha_{f}\left(x_{3}-H / 2\right)},
$$

where $\alpha_{f}=\sqrt{\left(\zeta_{f} / \zeta\right)^{2}-1}, \beta_{f}$ is an unknown amplitudes of the waves in the upper halfspace fluid and $\zeta_{f}$ is the wavenumber of bulk waves in the fluid. Since viscosity of the fluid is not considered $T_{13}^{f}=T_{23}^{f}=0$. The electric potential for a dielectric fluid satisfies the condition $\phi_{, k k}^{f}=0$. A solution of this differential equation for a wave traveling in the $x_{1}-x_{3}$ plane can be expressed as:

$$
\hat{\phi}^{f}=\Phi^{f} e^{-\zeta\left(x_{3}-H / 2\right)},
$$

where, $\Phi^{f}$ is an arbitrary constant. The sign of the exponential must be chosen such that the electric potential vanishes towards infinite. The electric displacement for the fluid can be written as:

$$
\hat{D}_{3}^{f}=\varepsilon^{f} \zeta \Phi^{f} e^{-\zeta\left(x_{3}-H / 2\right)},
$$

where $\varepsilon^{f}$ is the electric permittivity of the fluid.

The continuity condition between layers implies that the displacements, stresses and electrical parameters must be continuous at the interfaces. A well known method to satisfy these conditions is the use of the transfer matrix; which relates the displacements and stresses of the top and bottom surfaces of the plate (Nayfeh, 1995)

$$
P^{+}=A P^{-}
$$

where $P^{+}=\left\{\hat{u}, \hat{w}, \hat{\phi}, \hat{T}_{33}, \hat{T}_{13}, \hat{D}_{3}\right\}_{x_{3}=H / 2}, P^{-}=\left\{\hat{u}, \hat{w}, \hat{\phi}, \hat{T}_{33}, \hat{T}_{13}, \hat{D}_{3}\right\}_{x_{3}=-H / 2}, H$ is total thickness of the plate, and $A$ is a $6 \times 6$ transfer matrix in terms of the elastic properties and dimensions of the layered plate (Appendix C).

\subsubsection{Boundary Conditions}

The boundary conditions can be easily treated using transfer matrix approach. The displacements and stresses at the top surface of the plate must match those of the fluid 


$$
P^{+}=\left\{\begin{array}{c}
\hat{u}_{1} \\
\hat{u}_{3} \\
\hat{\phi} \\
\hat{T}_{33}^{*} \\
\hat{T}_{13}^{*} \\
\hat{D}_{3}^{*}
\end{array}\right\}_{x_{3}=H / 2}=\left\{\begin{array}{c}
\beta_{f} \\
-\alpha_{f} \beta_{f} \\
\Phi^{f} \\
\rho^{f} \omega^{2} / \zeta^{2} \\
0 \\
-i \varepsilon^{f} \Phi^{f}
\end{array}\right\}
$$

where $\hat{T}_{33}^{*}=\hat{T}_{33} / i \zeta$ and $\hat{D}_{3}^{*}=\hat{D}_{3} / i \zeta$. The displacements and stresses at the bottom of the plate are the same of those of the half-space solid

$$
P^{-}=\left\{\begin{array}{c}
\hat{u}_{1} \\
\hat{u}_{3} \\
\hat{\phi} \\
\hat{T}_{33}^{*} \\
\hat{T}_{13}^{*} \\
\hat{D}_{3}^{*}
\end{array}\right]_{x_{3}=-H / 2}=\left[\begin{array}{lll}
P_{11} & P_{12} & P_{13} \\
P_{21} & P_{22} & P_{23} \\
P_{31} & P_{32} & P_{33} \\
P_{41} & P_{42} & P_{43} \\
P_{51} & P_{52} & P_{53} \\
P_{61} & P_{62} & P_{63}
\end{array}\right]\left\{\begin{array}{l}
\beta_{1} \\
\beta_{2} \\
\Phi_{s}
\end{array}\right\}
$$

where the matrix $[P]$ is equivalent to the transfer matrix, but derived for the half-space (Appendix C), and the parameters $\beta_{i}$ and $\Phi_{s}$ are the amplitudes of the partial waves in the solid half-space. Since radiation of energy from the plate to the half-space solid is expected, two partial waves which have increasing amplitude with depth are the only ones present in the half-space solid; therefore, the amplitude $\beta$ of the other two partial waves must be equal to zero. Substituting Eq. (5.8) and (5.9) in (5.7), the following system of equations is obtained

$$
\left\{\begin{array}{l}
0 \\
0 \\
0 \\
0 \\
0
\end{array}\right\}=\left[\begin{array}{ccccc}
Q_{21} & Q_{22} & Q_{23} & \alpha_{f} & 0 \\
Q_{31} & Q_{32} & Q_{33} & 0 & -1 \\
Q_{41} & Q_{42} & Q_{43} & -\rho^{f} \omega^{2} / \zeta^{2} & 0 \\
Q_{51} & Q_{52} & Q_{53} & 0 & 0 \\
Q_{61} & Q_{62} & Q_{63} & 0 & i \varepsilon^{f}
\end{array}\right]\left\{\begin{array}{c}
\beta_{1} \\
\beta_{2} \\
\Phi_{s} \\
\beta_{f} \\
\Phi_{f}
\end{array}\right\}
$$

where $Q_{i j}$ are the elements of the matrix result of the product of the transfer matrix $[A]$ and the $[P]$ matrix of Eq. (5.9). The dispersion relations can be obtained, for a given $\omega$, by finding the values of $\zeta$ for which the determinant of $5 \times 5$ matrix $[M]$ in Eq. (5.10) is equal to zero. This process can be carried out using Muller's method. From the dispersion relations, a series of useful parameters can be calculated, such as phase and group velocity and attenuation:

$$
\begin{gathered}
\mathrm{v}_{p}=\frac{\omega}{\operatorname{Re}(\zeta)}, \\
\mathrm{v}_{g}=\frac{d \omega}{d(\operatorname{Re}(\zeta))},
\end{gathered}
$$




$$
\text { and } \quad \gamma=\frac{\operatorname{Im}(\zeta)}{\operatorname{Re}(\zeta)}
$$

\subsubsection{Frequency Response to an External Force}

In this section a novel analytic procedure to obtain the resonance frequencies, based on the transient response structure, is developed. The displacement spectra of the laminate plate bonded to a half-space solid can be calculated using inverse Fourier transformation. First, the amplitudes of the partial waves in the fluid and the solid halfspace are obtained after considering a force excitation at the transducer top surface

$$
\left\{\begin{array}{c}
0 \\
0 \\
0(\omega, \zeta) \\
0
\end{array}\right\}=\left[\begin{array}{ccccc}
Q_{21} & Q_{22} & Q_{23} & \alpha_{f} & 0 \\
Q_{31} & Q_{32} & Q_{33} & 0 & -1 \\
Q_{41} & Q_{42} & Q_{43} & -\rho^{f} \omega^{2} / \zeta^{2} & 0 \\
Q_{51} & Q_{52} & Q_{53} & 0 & 0 \\
Q_{61} & Q_{62} & Q_{63} & 0 & i \varepsilon^{f}
\end{array}\right]\left\{\begin{array}{c}
\beta_{1} \\
\beta_{2} \\
\Phi_{s} \\
\beta_{f} \\
\Phi^{f}
\end{array}\right\} .
$$

The vector of unknown amplitudes can now be obtained by solving Eq. (5.14):

$$
\{\beta\}=\frac{1}{\operatorname{det}[M]} \operatorname{adj}[M]\{F\},
$$

where $\{F\}$ is the impulse vector (left hand side of Eq. 5.14) and $\operatorname{adj}[M]$ is the adjugate matrix of $[M]$. The displacements on the top of the plate can be obtained using Eq. (5.9) and the results from Eq. (5.15). The displacements response at a certain point $x_{1}$ can be obtained by the inverse transformation:

$$
\bar{\varphi}\left(x_{3}, x_{1} ; \omega\right)=\frac{1}{2 \pi} \int_{-\infty}^{\infty} \hat{\varphi}\left(x_{3} ; \zeta, \omega\right) e^{i \zeta x_{1}} d \zeta
$$

The integration of Eq. (5.16) is a complex integration that must be carried out over half of the complex plane. The residue theorem states that the integral is equal to $2 \pi i$ multiplied by the summation of the residues enclosed by the path. Both of $u_{i}$ and $\phi$ have a series of singularities when det $[M]=0$. However, these singularities are poles of first order since the singularity vanishes when Eq. (5.15) is multiplied by $\left(\zeta-\zeta_{n}\right)$. Therefore, the residues for displacements normal to the top of the plate can be defined as:

$$
\operatorname{Res}\left(\hat{u}_{3}, \zeta_{n}\right)=2 \pi i \sum_{q=1,2} Q_{2 q} \beta_{q}^{*} e^{i \zeta_{n} x_{1}}
$$

where,

$$
\beta_{q}^{*}=\operatorname{Lim}_{\zeta \rightarrow \zeta_{n}} \frac{\left(\zeta-\zeta_{n}\right)}{\operatorname{det}[M]} \operatorname{adj}[M]\{F\} .
$$

The displacement frequency response at the top of the plate can now be written as:

$$
\bar{u}_{3}\left(x_{1} ; \omega\right)=i \sum_{n=1}^{N} \sum_{q=1,2} Q_{2 q} \beta_{q}^{*} e^{i \zeta_{n} x_{1}},
$$

where $N$ is the number of poles considered in the integration. Pan and Datta [31] determined that accurate results can be obtained with $40 \leq N \leq 60$. Choosing the displacement response at the point of excitation $\left(x_{1}=0\right)$, Eq. (5.19) can be expressed as

$$
\bar{u}_{3}(\omega)=i \sum_{n=1}^{N} \sum_{q=1,2} Q_{2 q} \beta_{q}^{*} \text {. }
$$


Equation (5.20) represents the frequency response of the displacement and electric potential due to an external force. If an impulse force is considered, the resonance frequencies can be obtained by plotting the amplitude of the displacement vs. frequency.

\subsection{Experimental Validation}

To validate the results of the analytical model, the resonance frequencies of PZT$5 \mathrm{H}$ plates with and without backing layer were obtained experimentally. The experimental setup shown in Figure 5.2 was used to obtain the resonance frequencies by measuring the displacement response of the piezoelectric plate after an electric impulse excitation. A function generator (Model AFG3102, Tektronix Inc., Beaverton, OR) was used to apply an electric impulse to plate with voltage amplitude of $10 \mathrm{~V}$ and duration of $1 \mu \mathrm{s}$. Then, the displacements in the normal direction of the plate were measured using a Nd:YAG interferometer (Model Tempo, Bossa Nova Technologies, Venice, CA). The data was acquired using an oscilloscope (Model TDS 2024B Tektronix Inc., Beaverton, OR). The resonance frequencies were obtained by calculating the Fast Fourier Transformation (FFT) of the time domain signal.

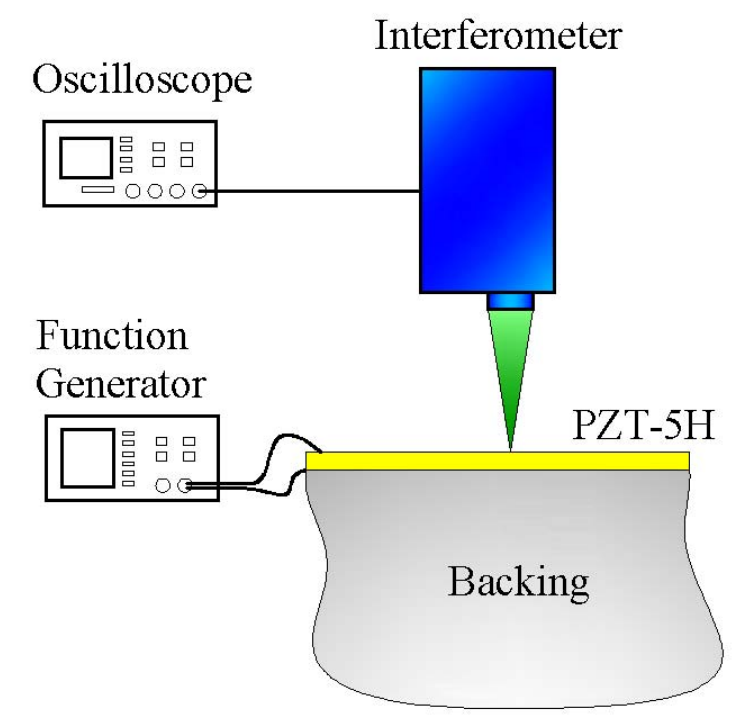

Figure 5.2. Experimental setup to measure the resonance frequencies of piezoelectric plates with and without backing layer.

\subsection{Results and Discussion}

The dispersion relations for the structures considered in this study can be obtained by solving Eqs. (5.10). This means that for a given value of the frequency $\omega$ there are several values of the wavenumber $\zeta$ that satisfy the conditions expressed in Eq. (5.10). Once the dispersion relation is determined, the wave attenuation and velocities can be obtained. The dispersion curves for several transducer configurations are compared to evaluate the effect of backing layer, matching layers and fluid loading on the dynamic behavior of these devices. The results in this study are presented using non-dimensional frequency $\left(\Omega=\omega\left(h \sqrt{\rho / C_{44}}\right)\right)$ and non-dimensional wavenumber $(\mathrm{K}=\zeta h)$, where $h, \rho$ 
and $C_{44}$ are the thickness, density and shear modulus of the piezoelectric plate, respectively.

\subsubsection{Validation}

To validate the present analytical formulation, results from two special cases reported by Achenbach and Kasheva (1967) and Nayfeh and Chimenti (1984) were adopted. First, an elastic isotropic plate bonded to an isotropic half-space was considered (Achenbach and Keshava, 1967). The ratio between the mass densities $\rho_{L} / \rho_{h s}$ was set equal to 0.75 , the ratio between the shear moduli $G_{L} / G_{h s}$ was 0.1 and the Poisson's ratio was 0.25 for both materials. The comparison for the phase velocity vs wavenumber for the first four modes shows an excellent agreement between the formulations (Fig. 5.3). Second, a comparison between experimental and theoretical results of Rayleigh wave propagation in a steel half-space coated with chromium and surrounded by water on top was considered. The densities and mechanical properties are presented in Table 5.1. A very good agreement between the present formulation and the theoretical and experimental results reported by Nayfeh and Chimenti (1984) was observed (Fig. 5.4).

Table 5.1a. Mechanical properties for the materials used in the analysis.

\begin{tabular}{|c|c|c|c|c|c|c|c|c|}
\hline \multirow[t]{2}{*}{ Material } & \multicolumn{6}{|c|}{$\begin{array}{c}\text { Elastic Properties } \\
(\mathrm{GPa})\end{array}$} & \multirow{2}{*}{$\begin{array}{c}\text { Density } \\
\left(\mathrm{Kg} / \mathrm{m}^{3}\right) \\
\rho\end{array}$} & \multirow[t]{2}{*}{$\begin{array}{c}\text { Impedance } \\
\text { (MRalys) }\end{array}$} \\
\hline & $C_{11}$ & $C_{12}$ & $C_{13}$ & $C_{33}$ & $C_{44}$ & $C_{66}$ & & \\
\hline PZT-5H & 120 & 75.2 & 75.1 & 111 & 21.1 & 22.6 & 7750 & 30.5 \\
\hline PZT-4 & 139 & 77.8 & 74.3 & 115 & 25.6 & 30.6 & 7500 & 32.3 \\
\hline Steel & 255 & 103 & 103 & 255 & 76 & 76 & 7900 & 46.6 \\
\hline Chromium & 313 & 83 & 83 & 313 & 115 & 115 & 7200 & 47.5 \\
\hline Backing Layer 1 & 7.98 & 5.18 & 5.18 & 7.98 & 1.4 & 1.4 & 1130 & 3.0 \\
\hline Backing Layer 2 & 13.0 & 5.6 & 5.6 & 13.0 & 3.7 & 3.7 & 3888 & 7.1 \\
\hline Matching Layer 1 & 26.0 & 10.0 & 10.0 & 26.0 & 8.0 & 8.0 & 2889 & 8.6 \\
\hline Matching Layer 2 & 4.64 & 1.64 & 1.64 & 4.64 & 1.5 & 1.5 & 1160 & 2.3 \\
\hline
\end{tabular}

Table 5.1b. Piezoelectric properties for PZT-4.

\begin{tabular}{l|ccccc}
\hline \multirow{2}{*}{ Material } & \multicolumn{2}{|c}{ Piezoelectric Stress Constant } & \multicolumn{3}{c}{ Clamped Dielectric } \\
& \multicolumn{2}{c}{$\left(\mathrm{C} / \mathrm{m}^{2}\right)$} & $e_{33}$ & $\varepsilon_{33}$ & $\varepsilon_{11}$ \\
\hline PZT-5H & $e_{15}$ & $e_{31}$ & 15.8 & 830 & 916 \\
\hline PZT-4 & 12.3 & -5.4 & 15.1 & 635 & 730 \\
\hline
\end{tabular}

The effect of the backing layer on the resonance frequency for a piezoelectric plate has been studied experimentally and analytically. First, the resonance frequency for a PZT-5H with and without backing was obtained following the procedure described above. Figure 5.5 a shows a reduction of $9.1 \%$ of the resonance frequency when a backing layer of acoustical impedance of 3 MRayls (Table 5.1) is bonded to the piezoelectric plate. This difference is important for the selection of the operation frequency and the design of matching layers for the transducer. The resonance frequencies, obtained using the analytical model, were also validated and compared with experimental measurements. Figure $5.5 \mathrm{~b}$ shows the comparison of the resonance 
frequencies for a PZT-5H plate bonded to a 3.0 MRayls backing layer. The difference between analytical prediction and experimental values was $5.8 \%$ and $4.7 \%$ for the first and second resonance frequencies, respectively. This comparison shows a good agreement between the analytical analysis presented here and experimental measurements.

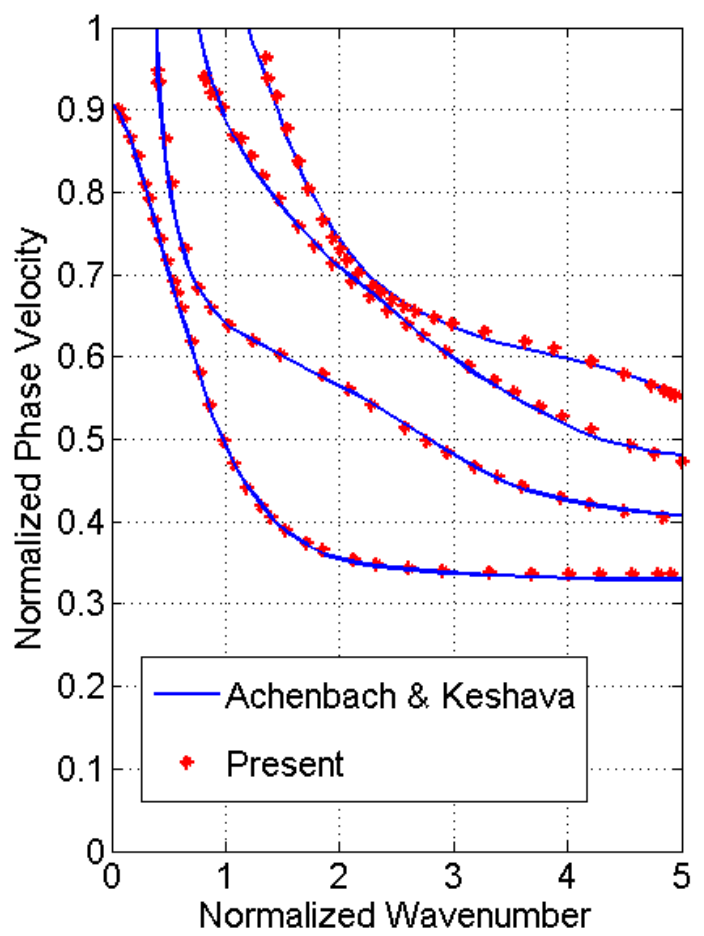

Figure 5.3. Validation of the model: Comparison of the analytical results for the first four modes presented by Achenbach and Keshava (1967) for an elastic layer on top of an elastic half-space.

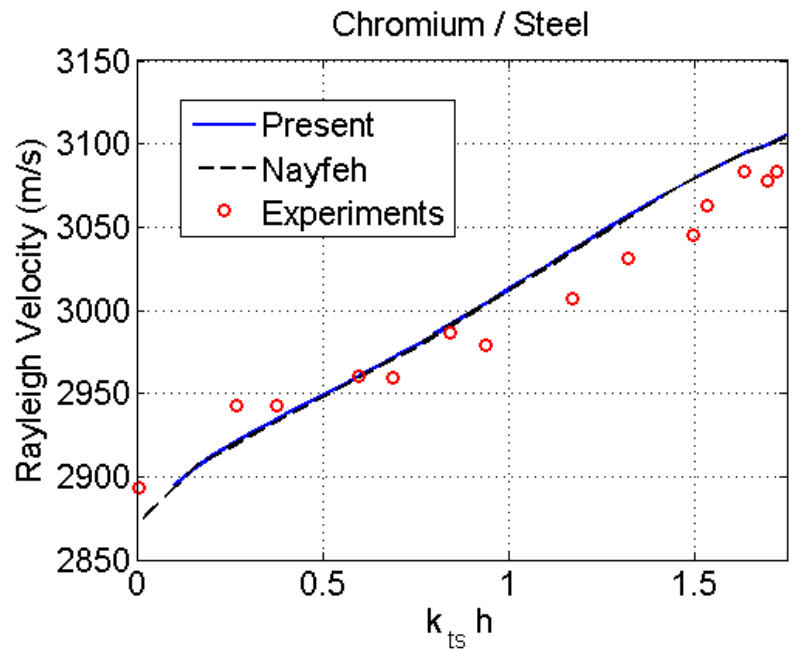

Figure 5.4. Validation of the model: Comparison between the analytical and experimental results reported by Nayfeh and Chimenti (1984) for a chromium layer surrounded by a half-space solid (steel) and a half-space fluid (water). $k_{\mathrm{ts}}$ is wavenumber of the shear mode of the substrate and $h$ is the thickness of the chromium layer. 


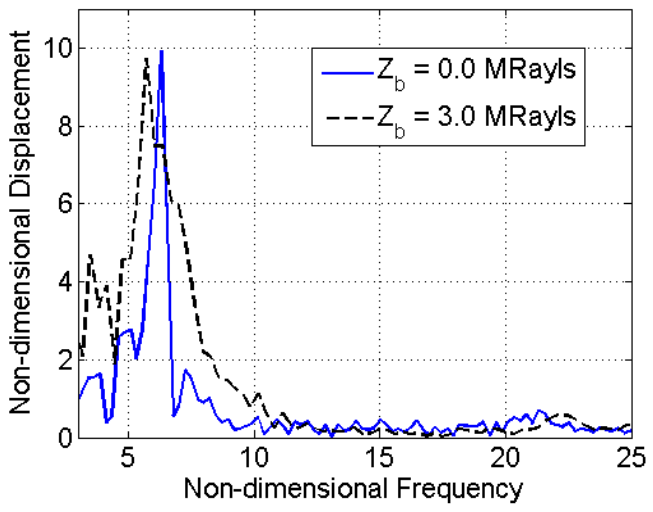

a)

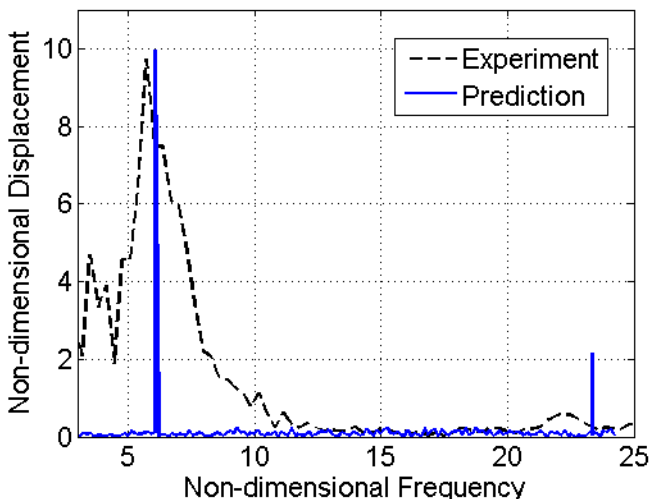

b)

Figure 5.5. Effect of backing layer on the resonance frequencies of a piezoelectric plate. a) comparison between experimental resonance frequencies for a PZT-5H plate with and without backing layer, and b) comparison between experimental and analytical prediction of the resonance frequencies of PZT-5H plate on a backing layer.

\subsubsection{Effect of the backing layer on the dispersion curves and resonance frequencies}

Once the analytical formulation has been validated, the effect of the backing and matching layers on the dispersion curves and resonance frequencies can be now studied analytically. First, the dispersion curves for a PZT-4 plate bonded to an elastic halfspace, representing the backing layer (7.1 MRayls), are compared to those obtained for the PZT-4 plate in vacuum or air (Fig. 5.6a). The dispersion curves for the $\mathrm{S}_{0}$ and $\mathrm{A}_{0}$ modes present small variations compared with plate in vacuum or air. The dashed line of the $\mathrm{A}_{0}$ mode corresponds to the non-radiating portion of this mode; details about radiation are given below. The $A_{1}$ and $S_{2}$ modes exist at lower frequencies and their cutoff frequency has been considerably reduced. Figure $5.6 \mathrm{~b}$ shows the trajectory on the complex plane of the first five modes. It can be observed that, for the piezoelectric plate bonded to a backing layer, the wavenumber for all the modes are complex values, even at the cut-off frequencies when Real $(K)=0$. On the contrary, for the plate in vacuum the wavenumbers are real values, except for the $S_{1}$ mode which is complex for low frequencies $(\Omega<6.0)$ and becomes real for frequencies larger than the zero-groupvelocity $(\mathrm{ZGV})$ point. 


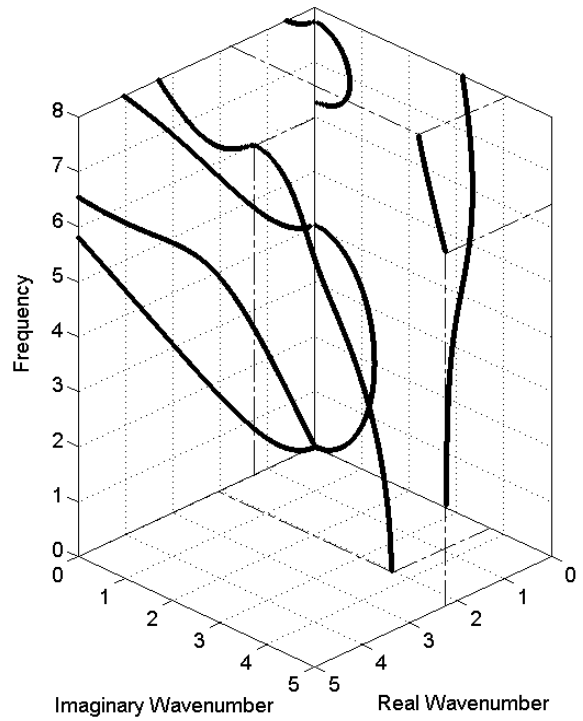

a)

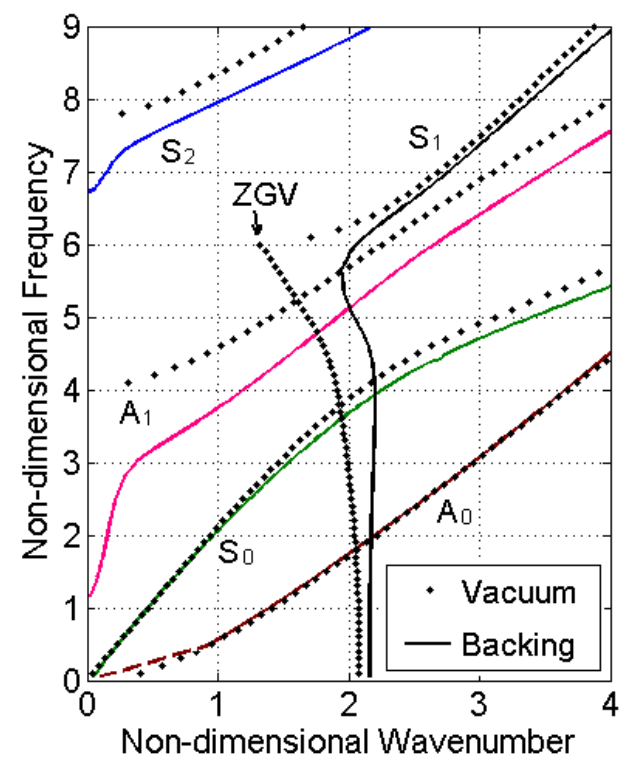

c)

Figure 5.6. Comparison between a PZT-4 surrounded by air and bonded to a half-space solid representing a backing layer: a) Three-dimensional dispersion curves for a PZT-4 in vacuum, b) Three-dimensional dispersion curves for PZT-4 plate bonded to a backing layer (7.1 MRayls), c) Comparison between two-dimensional dispersion curves, d) comparison between the trajectory of all the modes in the complex plane.

The attenuation of the first five modes for the PZT-4 plate bonded to the backing layer (7.1 MRayls) is presented in Fig. 5.7. The phase velocity of all the modes, except a small portion of the $\mathrm{A}_{0}$ mode at low frequency $\Omega(<0.81)$, is larger than the shear velocity of the backing layer; therefore, acoustic waves are radiated from plate to the backing layer. On the other hand, when the phase velocity of the $\mathrm{A}_{0}$ mode is smaller than the phase velocity of the substrate, there is not radiation of energy to the backing layer 
and the displacements in the substrate decay with depth, similar to Rayleigh waves. The radiation of acoustic waves implies that the waves propagating in the plate are gradually losing energy and amplitude with distance. This effect is known as attenuation and can be calculated from the dispersion relations by Eq. (5.13). It can be observed that both $\mathrm{A}_{0}$ and $\mathrm{S}_{0}$ modes have low attenuation in the considered range of frequencies. The $\mathrm{A}_{0}$ mode is not attenuated when its phase velocity is lower than the shear velocity of the backing layer at frequency $\Omega(<0.81)$. The $\mathrm{S}_{1}$ mode exhibits a very high attenuation behavior at low frequency $\Omega(<6.3)$. An interesting behavior is observed for the $A_{1}$ and $S_{2}$ modes that they have infinite attenuation component although the imaginary component of the wavenumber is finite at cut-off frequency (i.e., $\operatorname{Real}(\mathrm{K})=0$ ). However, the ratio between the imaginary and real parts of the wavenumber tends to infinity. The attenuation of these modes decreases to values similar to those of propagating modes $\left(\mathrm{A}_{0}\right.$ and $\left.\mathrm{S}_{0}\right)$ after a certain frequency denoted as 'transition frequency'. On the other hand, the $\mathrm{S}_{1}$ mode has a finite attenuation component at low frequency; however, there is a significant reduction of the attenuation occurs as the frequency increases beyond the transition frequency. The transition frequency can be defined qualitatively as the frequency for which the attenuation reduces to values similar to those of the propagating modes $\left(\mathrm{A}_{0}\right.$ and $\left.\mathrm{S}_{0}\right)$. Physically, this frequency can be interpreted as the frequency for which a given mode becomes propagating; similar to the concept of cut-off frequency for plates in vacuum. A quantitative definition can be given for some modes as the frequency for which the magnitude of the wavenumber $(|K|)$ reaches a minimum value. The $A_{1}$ and $S_{1}$ modes have a minimum magnitude of the wavenumber at wave frequency $\Omega(=3.26$, and 6.3$)$, respectively. If these two points are marked in the attenuation curves (Fig. 5.7), it can be observed that they are related to transition from high to low attenuation.

It is important to notice that there exists a relation between the resonance frequencies and the frequencies for which the wavenumber has a minimum magnitude (transition frequency). Figure 5.8 shows the displacement response and the magnitude of the wave for the corresponding modes for a PZT-4 plate bonded to a backing layer of 3.0 MRayls. It is evident that the resonance frequencies are associated with the frequencies for which the absolute value of the wavenumber reaches a minimum (i.e., transition frequencies). This relation shows the importance of the dispersion curves on the dynamic behavior of these structures.

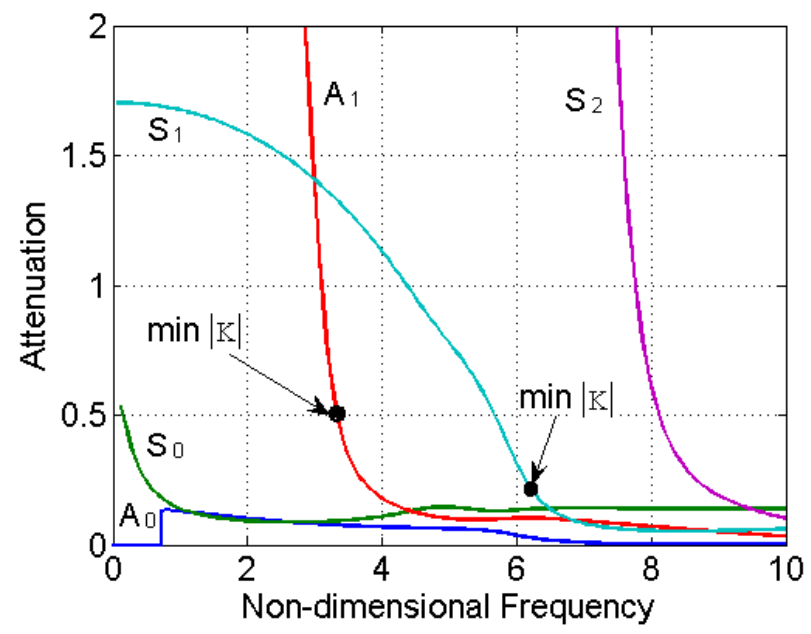


Figure 5.7. Attenuation spectra of the first five modes for a PZT-4 plate bonded to a backing layer $\left(\mathrm{Z}_{\mathrm{b}}=7.1\right.$ MRayls). Transition frequencies for $\mathrm{A}_{1}$ and $\mathrm{S}_{1}$ modes are presented by $(\min |\mathrm{K}|)$ points.

The effect of the backing layer on the resonance frequencies can be observed in Fig. 5.9. Comparing the displacement response of a plate in vacuum and one bonded to backing layers, it can be observed that the resonance frequencies associated with the $\mathrm{S}_{1}$ and $\mathrm{S}_{4}$ modes are very similar ( $\sim 2 \%$ difference). This observation may suggest that these resonance frequencies are mainly influenced by the mechanical behavior of the piezoelectric plate. On the other hand, the resonance points associated with the $A_{1}$ and $\mathrm{A}_{2}$ modes were not observed for the plate without backing layer; therefore, they must be related to the mutual interaction between the plate and the backing layer.

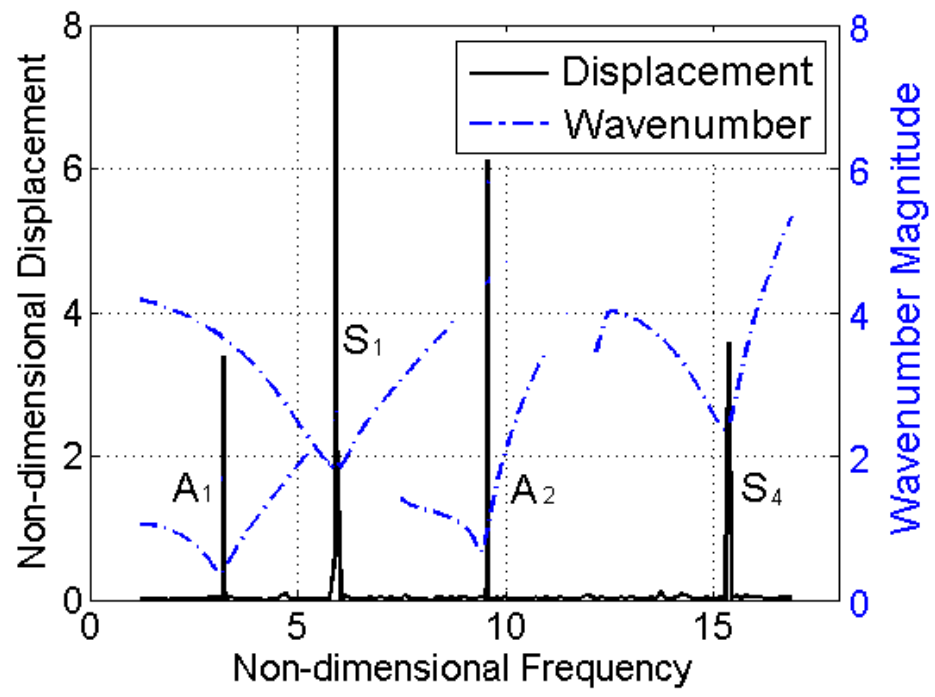

Figure 5.8. Relation between the resonance frequencies and the minimum magnitude of the wavenumber for a PZT-4 plate with a backing layer $\left(Z_{b}=3.0\right.$ MRayls $)$.

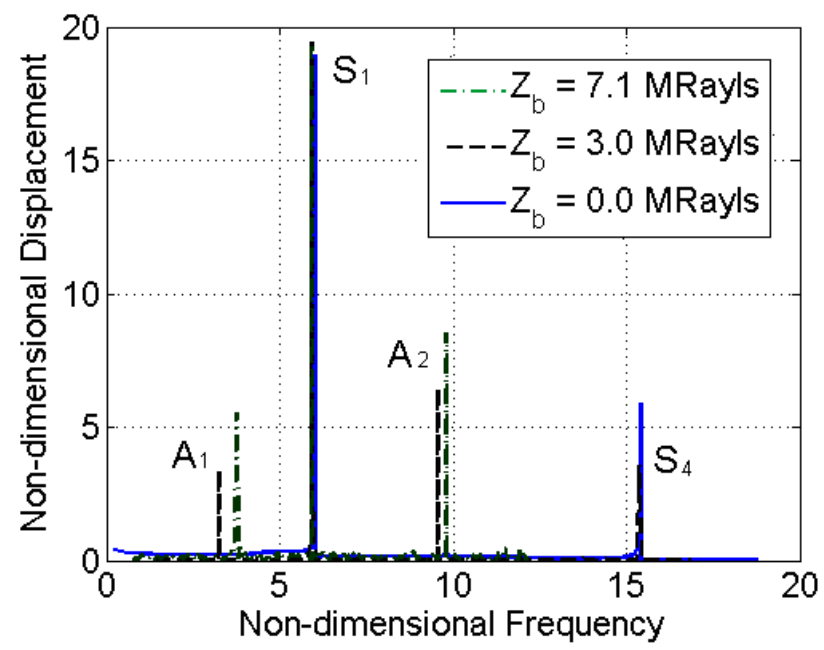

Figure 5.9. Comparison of the first two resonance frequencies for a PZT-4 plate with and without backing layer. 


\subsubsection{Effect of fluid loading on the dispersion curves and resonance frequencies}

The effect of fluid-loading on the characteristics of wave propagation is also studied. A comparison between the dispersion curves for air/vacuum and water loading on a PZT-4 plate bonded to a backing layer (7.1 MRayls) is presented in Fig. 5.10. It can be observed that the difference on the dispersion curves is small especially for the lowest modes. This indicates that the main changes in the dispersion curves are caused by the presence of the backing layer and no significant change will be observed on dynamic behavior of transducer when the fluid loading is considered. However, a change in attenuation for air and water loading was observed for low-order modes (Fig. 5.11). The attenuation increases approximately $30 \%$ at low frequencies for the $\mathrm{A}_{0}$ mode and about $15 \%$ at $\Omega=5$ for the $\mathrm{S}_{0}$ mode.

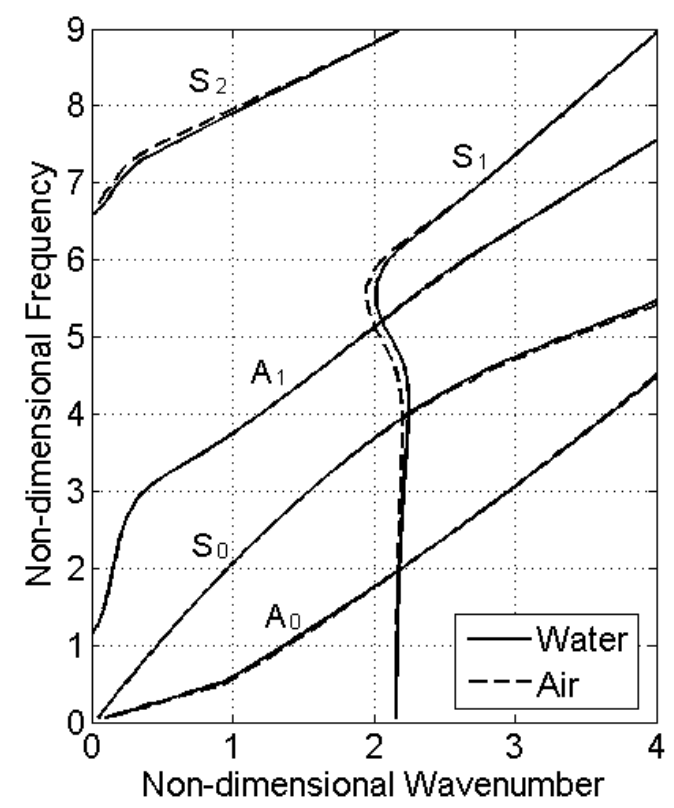

Figure 5.10. Comparison of the dispersion curves for a PZT-4 plate bonded to a backing layer $\left(Z_{b}=7.1\right.$ MRayls $)$ with air and water on top.

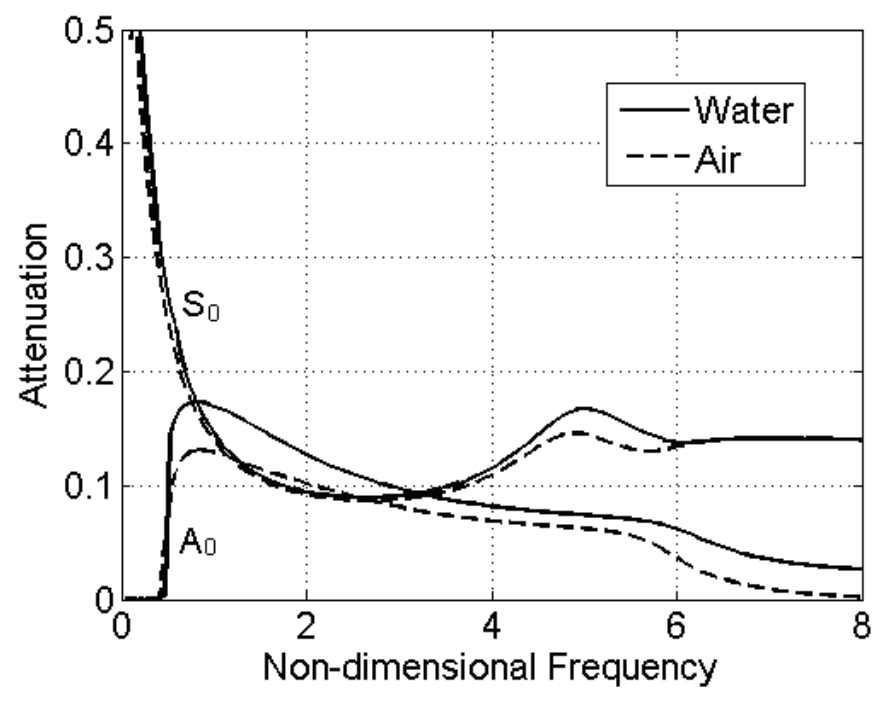


Figure 5.11. Comparison of the attenuation between water and air on top for a PZT-4 plate supported by a backing layer $\left(Z_{b}=7.1\right.$ MRayls $)$.

\subsubsection{Effect of matching layers on the dispersion curves and resonance frequencies}

The effect of matching layers has been also considered. The matching layers were designed following the KLM methodology for water loading. The mechanical properties are shown in Table 5.1. This configuration represents a fully functional immersion single-element transducer. The dispersion curves show an increase in the number of modes present in the frequency range considered (Fig. 5.12). Furthermore, the number of modes starting from the complex plane (highly attenuated at low frequencies) has increased. Comparing Figs. 5.6 and 5.12, it is noticed hat the matching layers decrease the cut-off frequencies and increase the number of propagating modes within the same frequency range. The attenuation for some of the modes is presented in Figure 5.13. It can be observed that the attenuation presents similar behavior than the previous cases. The relation between the resonance frequencies and the magnitude of the wavenumber can be observed in Figure 5.14. Again, the resonance frequencies are present at the frequency for which the magnitude of the wavenumber is minimum.

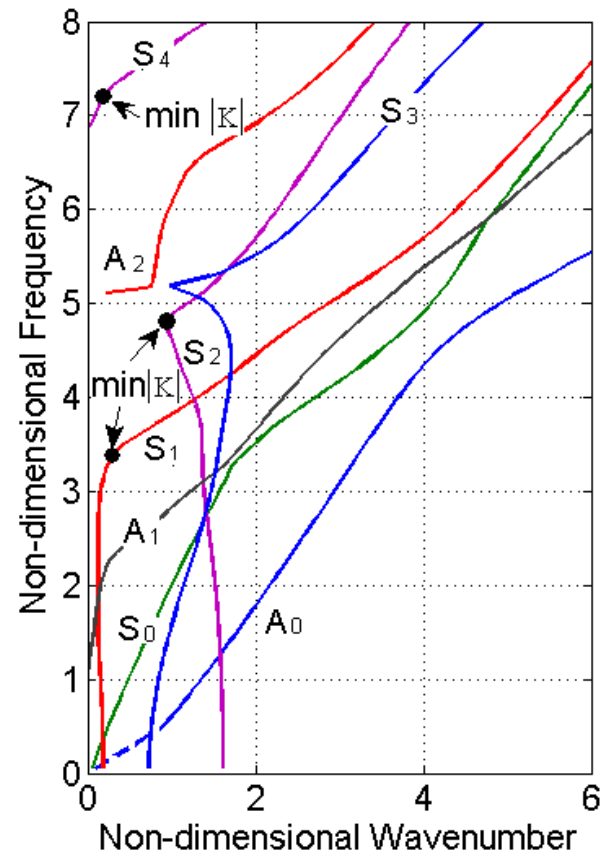

Figure 5.12. Dispersion curves for a PZT-4 transducer including backing layer (7.1 MRayls), matching layers (Table 5.1). 


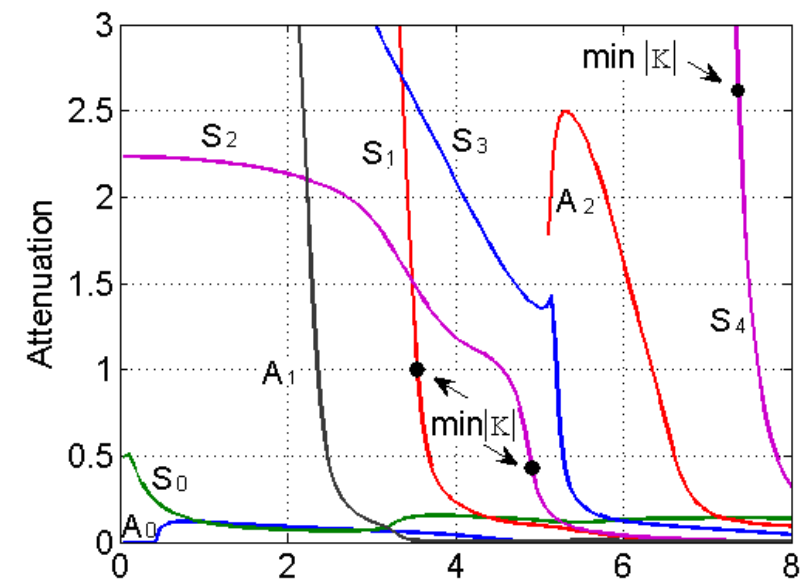

Figure 5.13. Attenuation of selected modes for a PZT-4 transducer with a backing layer (7.1 MRayls), matching layers (Table 5.1).

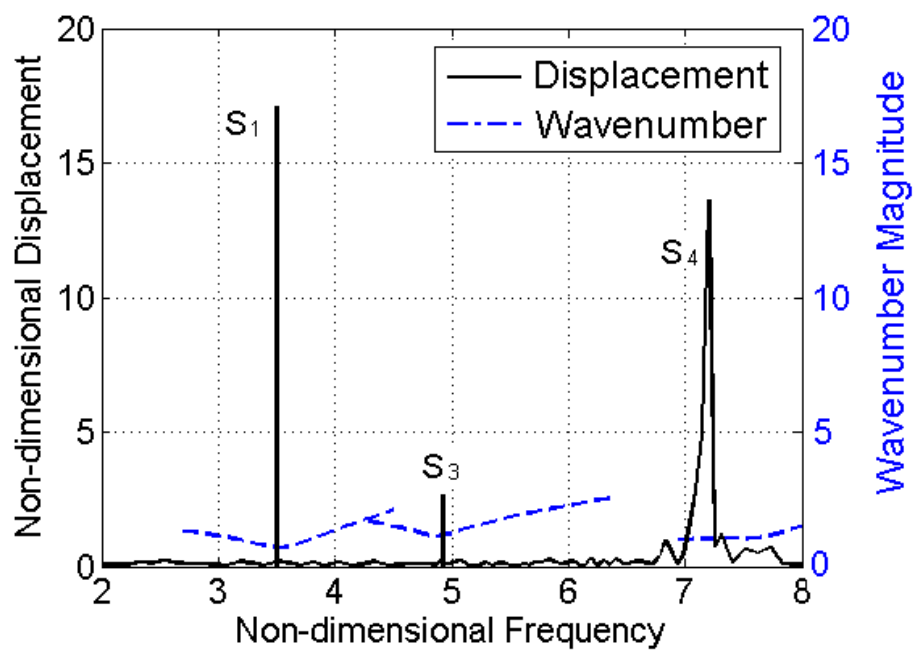

Figure 5.14. Comparison between the resonance frequencies and the magnitude of magnitude of the wavenumber for a PZT-4 transducer with a backing layer (7.1 MRayls), matching layers (Table 5.1).

\subsection{Conclusions}

An analytical model has been presented to study the dynamic behavior of singleelement ultrasound transducers. This model is based on leaky Lamb wave analysis, which has been widely used for NDT applications. A procedure to calculate the resonance frequencies for ultrasound transducers based on the dispersion curves has been derived and experimentally validated. The analysis of the effect of matching and backing layers showed that significant differences can be observed in the dispersion curves, attenuation and resonance frequencies. The addition of a backing layer lowered cut-off frequencies of the modes. It was also found that those modes have infinite attenuation at the cut-off frequency. However, the attenuation decreased to very low values after certain transition frequency. The transition frequency occur at minimum wavenumber values 
$(\min |\mathrm{K}|)$, and the wave displacements exhibit resonance behavior at these frequencies. This study would be useful for accurate characterization of multi-resonant frequencies of ultrasound transducers using leaky Lamb wave analysis. 


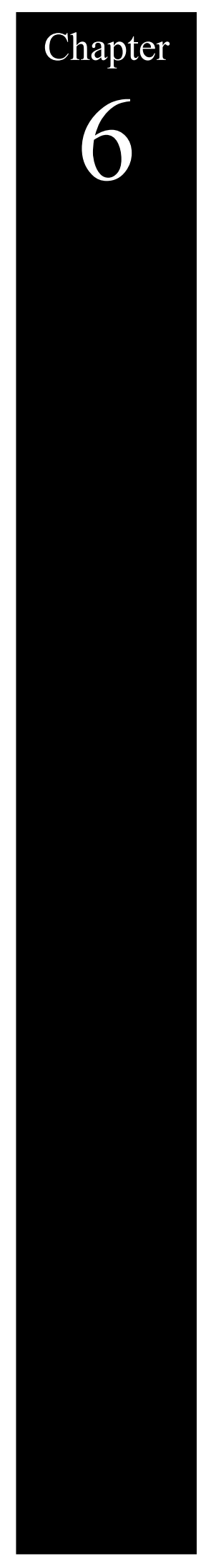

Modeling and Optimization of Acoustic Sensors 


\subsection{Introduction}

Many types of sensors have been designed and fabricated based on surface acoustic waves (SAW). The principle behind all these sensors is that a change in the mechanical properties (i.e. density) of the surrounding environment will produce a measurable variation of one or several parameters of the wave propagation. Some sensors have a special layer, which absorbs or attract substances or viruses causing a change in the mechanical properties of the layer and consequently alters the characteristics of the wave propagation (Gronewold, 2007). Other sensors do not change their properties, but the interaction with the surrounding fluid allows them to 'sense' a change in the fluid properties, such as density, viscosity, etc. These sensors are based on the propagation of 'leaky' surface acoustic waves (Lindner, 2008). Leaky waves are characterized by an exchange of energy between the solid and fluid, which produces a reduction or attenuation of the amplitude of the wave along the direction of propagation. The severity of this attenuation is function of the frequency and the properties of the fluid (Nayfeh, 1995). Therefore, for a given frequency, a change in the attenuation is an indication of a change of the fluid properties. In addition to attenuation, a reduction in the wave velocity may also be observed. Consequently, wave velocity can be also used as a probe to measure changes in the fluid.

Several types of surface waves have been applied in ultrasonic sensors (Ballentine et al., 1997). Rayleigh wave sensors are composed of one or several thin layers bounded to an elastic substrate. Rayleigh wave is a surface wave characterized by an elliptic displacement of the surface particles. This displacement is contained in the plane formed by the propagation direction and the axis normal to the surface (Auld, 1990). The out of plane component of the displacement produces a strong coupling between the surface and the bulk wave in the fluid. On the other hand, shear-horizontal surface waves are characterized by an oscillatory displacement parallel to the surface and perpendicular to the propagation direction (Auld, 1990). This implies that the out-of-plane component of the displacement is negligible or not present at all. This type of wave has been frequently used to measure of monitor changes in the viscosity of the fluid (Ricco and Martin, 1987). Usually the displacements of surface waves are confined to surface, i.e. the penetration depth of these waves is about one wave length.

When the thickness of the substrate is finite, i.e., the thickness of the substrate is comparable with the wave length, the propagated wave is called Lamb waves. Three modes of Lamb waves can be excited: symmetric, anti-symmetric and shear-horizontal. The displacements of these modes are similar to those of Rayleigh waves, but the amplitude is higher. Usually, for thin plates, only the lowest-order symmetric and antisymmetric modes ( $\mathrm{S}_{0}$ and $\mathrm{A}_{0}$, respectively) can be excited. In fact, these modes exhibit dispersion behavior, where the wave velocity is dependent on the wave frequency. For very high frequencies the wave velocity of these modes tends to the Rayleigh wave velocity. Sensors based on Lamb waves usually have a strong coupling with the surrounding fluid and a high sensitivity (Ballentine et al., 1997); consequently, they are preferred over Rayleigh wave sensors. 
Sensitivity is a property of the sensor related to its capacity to detect changes in its environment. Therefore, each type of sensor has a different measurement of the sensitivity (Abdollahi, et al., 2007; Duhamel et al., 2006; Wu et al., 2008; Wenzel and White, 2008). For instance, sensors based on a sensitive layer which attracts certain biological molecules or agents have a sensitivity defined as the shift in resonant frequency over the gain in mass. In this study, the sensor is meant to monitor variations of the density of the surrounding fluid using attenuation of phase velocity. Several studies have addressed the sensitivity of this type of sensors (Wenzel and White, 1988; White and Wenzel, 1988). A detailed description of the sensitivity will be presented below.

The design of ultrasonic sensors requires the determination of geometrical parameters such as thickness of the piezoelectric layer and width and spacing of the IDT (Fig. 6.1). The width and spacing of the IDT is very important since it determines the wavelength and therefore the operation frequency of the transducer. On the other hand, thickness of the piezoelectric plate is related to the wave velocity, coupling factor and attenuation of the transducer. These two parameters can be selected to optimize the sensitivity for a given application.

To design a sensor for maximum sensitivity, it is necessary to calculate the change on attenuation and phase velocity for Lamb waves as a function of the density or other fluid parameters (Dickherber et al., 2009; Wang et al., 2007). Usually, numerical methods such as Finite Elements are used to simulate the wave propagation on this type of structures (Abdollahi et al., 2007). However, they are time consuming and the analysis of the data is often complicated. In this study, the analytical solution presented in Chapter 5, which is valid for multilayered piezoelectric plates surrounded by a fluid, is used to calculate the attenuation and wave velocities directly in the frequency domain. A design procedure based on these results will also be presented.
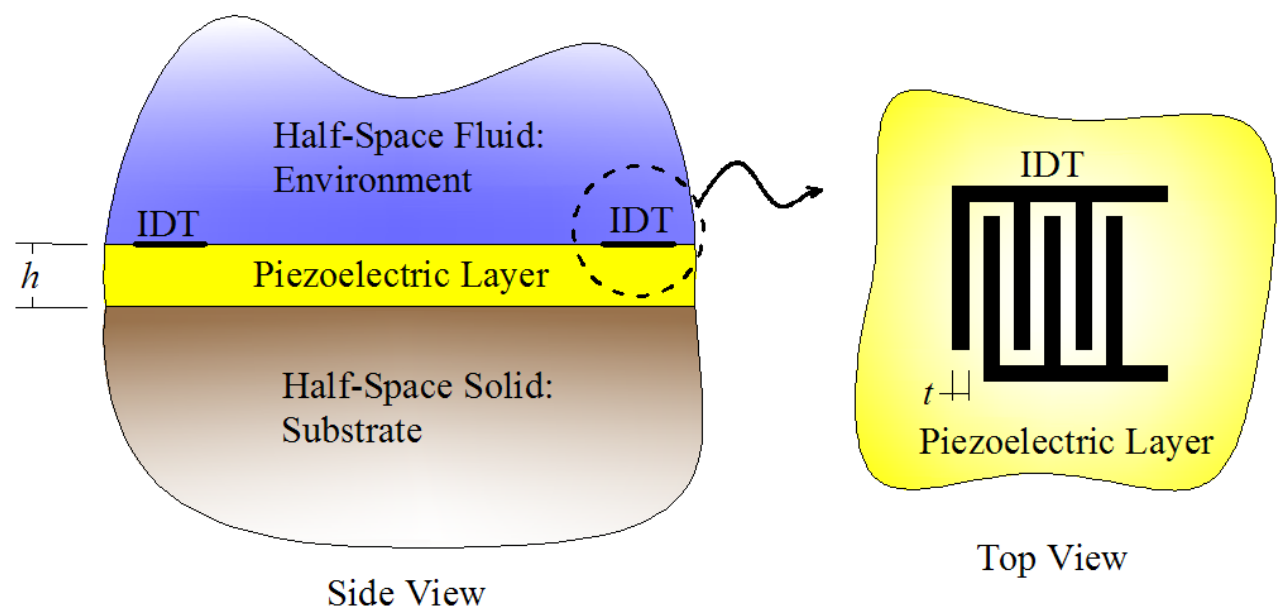

Figure 6.1. Geometrical parameters to be optimized for maximum sensitivity. 


\subsection{Model Description}

Rayleigh sensors are modeled as a multilayered infinite medium since the distance between IDTs is much larger than the thickness of the piezoelectric layer (Fig. 6.1). A piezoelectric plate is surrounded by a half-space solid and a half-space fluid on the other side representing the substrate and the environment, respectively. On the other hand Lamb wave sensors are modeled as a plate with fluid in one or both sides. The presence of fluid on the surface of the plate introduces several changes in the waves travelling between IDTs. Attenuation and reduction of the wave velocities are among the principal effects of the surrounding fluid.

The theoretical analysis of these sensors is based on the solution presented in Chapter 5, in which the constitutive relations and the motion equations are combined to find all the possible wave modes (partial waves) present on the plate (Nayfeh, 1995). The analytical formulation describes the procedure to obtain phase and group velocities and attenuation. The coupling coefficient and sensitivity can be obtained from the wave velocity and attenuation.

\subsection{Coupling Coefficient}

The coupling coefficient is a measurement of the piezoelectric effect on the sensor (Kallard, 1971). The presence of piezoelectricity increases the velocity of Rayleigh waves compared with the velocity it would have if only the elastic behavior were considered. This solution sometimes is called stiffened Rayleigh wave (Matthews, 1977). The effect of piezoelectricity can be partially 'switched-off' by short-circuiting the surface where the IDTs are located. The change in the Rayleigh wave velocity as a function of frequency can be observed in Fig. 6.2, for an Aluminum Nitride (AlN) layer bonded to a Silicon substrate (Table 6.1). Lower velocities are observed for the shortcircuit condition, especially at high frequencies.

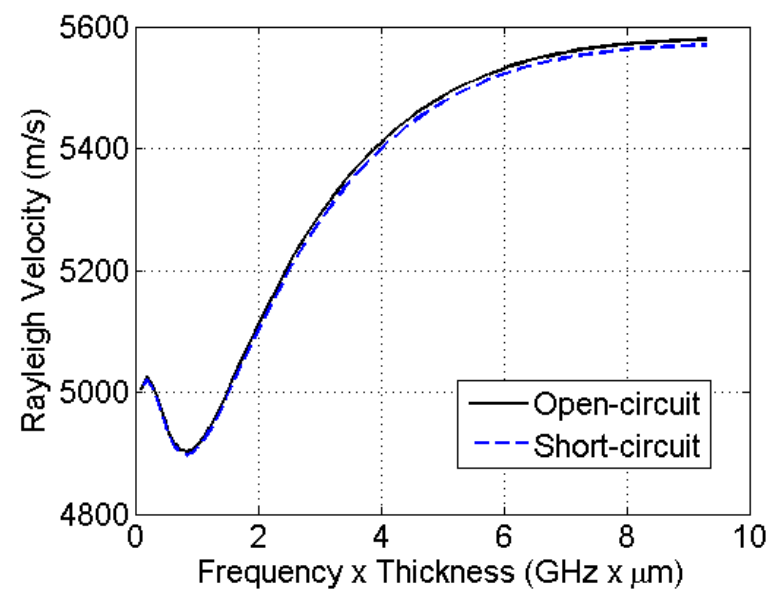

Figure 6.2. Comparison of the Rayleigh wave velocity for open-circuit and short-circuit boundary conditions for an AlN piezoelectric layer bonded to Silicon substrate. 
Table 6.1a. Mechanical properties for the materials used in the analysis.

\begin{tabular}{l|cccccccc}
\hline \multirow{2}{*}{ Material } & \multicolumn{4}{c}{$\begin{array}{c}\text { Elastic Properties } \\
(\mathrm{GPa})\end{array}$} & & $\begin{array}{c}\text { Density } \\
\left(\mathrm{Kg} / \mathrm{m}^{3}\right)\end{array}$ & $\begin{array}{c}\text { Impedance } \\
\text { (MRalys) }\end{array}$ \\
& $C_{11}$ & $C_{12}$ & $C_{13}$ & $C_{33}$ & $C_{44}$ & $C_{66}$ & $\rho$ & \\
\hline $\mathrm{AlN}$ & 345 & 125 & 120 & 395 & 118 & 110 & 3290 & 19.4 \\
$\mathrm{SiO}_{2}$ & 78.5 & 16.1 & 16.1 & 78.5 & 3.12 & 3.12 & 2200 & 13.1 \\
\hline
\end{tabular}

Table 6.1b. Piezoelectric properties for PZT-4.

\begin{tabular}{l|ccccc}
\hline \multirow{2}{*}{ Material } & \multicolumn{2}{|c}{ Piezoelectric Stress Constant } & \multicolumn{3}{c}{ Clamped Dielectric } \\
& $e_{15}$ & $e_{31}$ & $e_{33}$ & $\varepsilon_{33}$ & $\varepsilon_{11}$ \\
\hline $\mathrm{AlN}$ & -0.48 & -0.6 & 1.46 & 9.0 & 10.7 \\
\hline $\mathrm{SiO}_{2}$ & --- & --- & --- & 4.5 & 4.5 \\
\hline
\end{tabular}

A measurement of the piezoelectric effect on the wave velocity is defined by the coupling coefficient

$$
k^{2}=2 \frac{\Delta v}{v},
$$

where $\Delta v$ is the change in velocity due to the electrical shortening of the surface of the sensor. A high value of the coupling coefficient represents a high interaction efficiency of the wave with the electrodes (IDT) placed on the surface. This factor is important in the choice of substrate materials and layer dimensions for many surface wave devices. For a sensor composed of an AIN layer deposited on top a silicon substrate the coupling factor as a function of the product of frequency by thickness is shown in Fig. 6.3. It can be observed that the coupling coefficient is maximum close to $2.5 \mathrm{GHz} \cdot \mu \mathrm{m}$. This parameter can be used to select the optimum thickness at a given frequency.

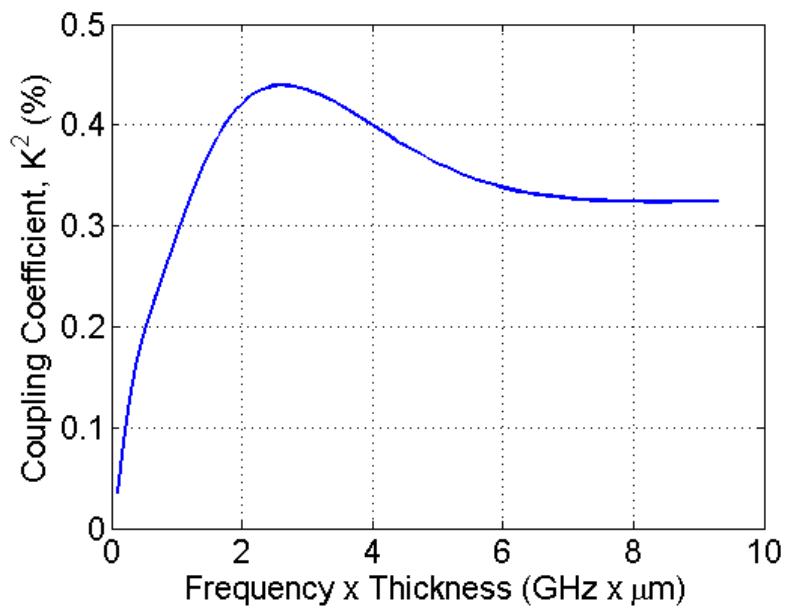

Figure 6.3. Coupling coefficient as function of the frequency and thickness of the AIN layer. 


\subsection{Wavelet Analysis}

The group velocity of Rayleigh and Lamb waves is associated with the energy carried by the wave. For Lamb waves the wave energy is distributed in all the propagating modes at a given frequency; and each of these modes travels at a different velocity. Therefore, the signal acquired by the receiving IDT is a combination of individual signals with different amplitudes and arriving times. The wavelet transformation of the acquired signal can provide useful information since it quantifies the amount of energy carried by each mode as a function of time and frequency (Kishimoto et al., 1995). This decomposition of the signal allows an easy identification of the modes and calculation of the group-velocities of the wave.

The wavelet transform used in this study was the Gabor wavelet which is based on the Gaussian function. Gabor Wavelet has been widely used in the field of acoustic emission because of the small window in the time as well as in the frequency domain that it can provide (Kishimoto et al., 1995; Wang and Yuan, 2007). The mother wavelet and its Fourier transform are given as:

$$
\begin{gathered}
\Psi(t)=\pi^{-\frac{1}{4}}\left(\frac{\omega_{p}}{\gamma}\right)^{\frac{1}{2}} \exp \left[-\frac{t^{2}}{2}\left(\frac{\omega_{p}}{\gamma}\right)^{2}+i \omega_{p} t\right], \\
\hat{\Psi}(\omega)=(2 \pi)^{\frac{1}{2}} \pi^{-\frac{1}{4}}\left(\frac{\omega_{p}}{\gamma}\right)^{\frac{1}{2}} \exp \left[-\frac{t^{2}}{2}\left(\frac{\omega_{p}}{\gamma}\right)^{2}\left(\omega-\omega_{p}\right)^{2}\right] .
\end{gathered}
$$

where $\omega_{p}$ is the center frequency and $\gamma$ is a constant taken as $\gamma=\pi(2 / \ln 2)^{1 / 2}=5.336$ (Jeong and Jang, 2000).

A Rayleigh wave sensor composed of an AlN layer with thickness of $900 \mathrm{~nm}$ bonded to a $\mathrm{SiO}_{2}$ substrate and with a distance between IDTs of $220 \mu \mathrm{m}$ was considered for this analysis. An impulse voltage of $20 \mathrm{~ns}$ and 10V was applied to the emitting IDT. This impulse signal was also used to trigger the data acquisition at the receiving IDT. The received signal was then transformed as explained above to obtain the arrival time. Figure $6.4 \mathrm{a}$ shows the impulse signal fed to the emitting IDT, Fig. 6.4b shows the acquired signal from receiving IDT and Fig. 6.4c shows the wavelet contours of the acquired signal. The dark red spot in the wavelet contour corresponds to the arrival time and frequency of the received signal $73 \mathrm{~ns}$. 

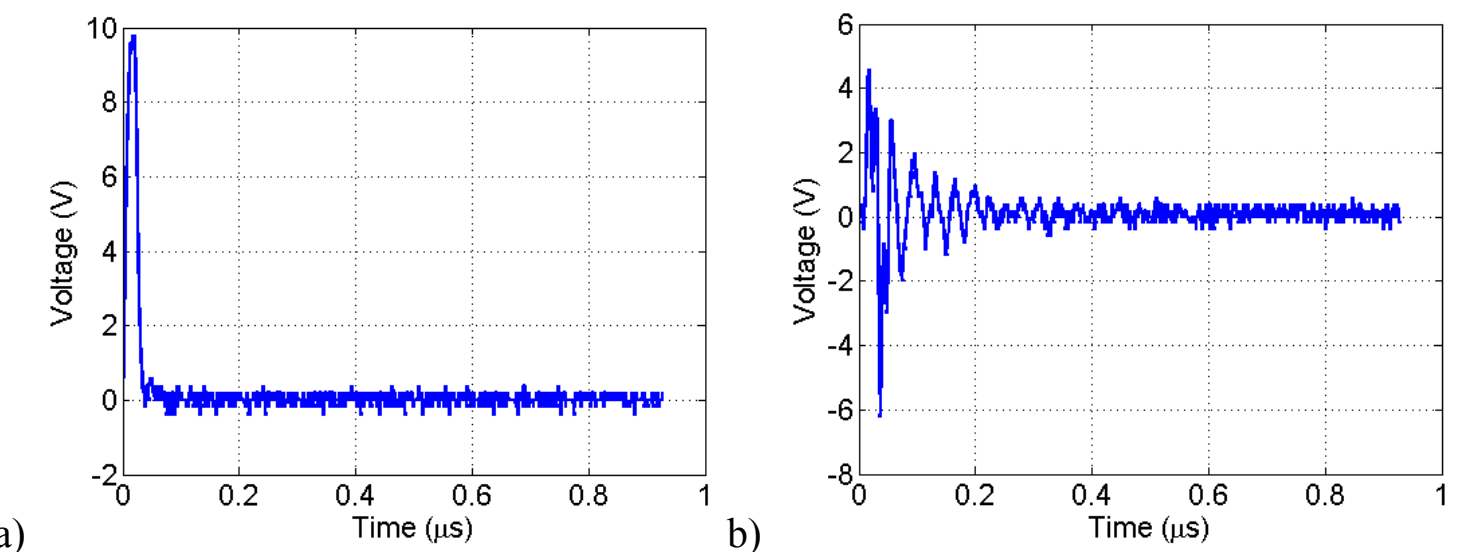

a)

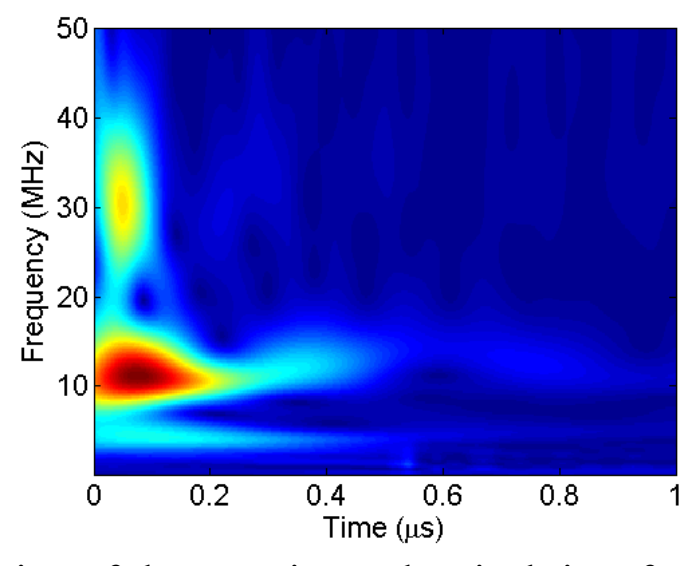

Figure 6.4. Determination of the experimental arrival time for a Rayleigh wave sensor using wavelet transformation: a) Impulse signal applied to the emitting IDT, b) Acquired signal from receiving IDT, c) wavelet contour of the acquired signal. The dark red spot indicates the frequency and arrival time of the wave.

To compare the measured values with those obtained using the analytical model, the group velocities as a function of frequency (Fig. 6.5a) were calculated using the analytical formulation. It can be observed from this figure that the group velocity presents a small change with an increase of the frequency. The theoretical arrival time can be calculated dividing the distance between IDTs by the group velocity (Fig. 6.5b). A comparison between theory and experiments can be observed in Fig. 6.5c, which shows a good agreement of the arrival time (difference 9.8\%). 

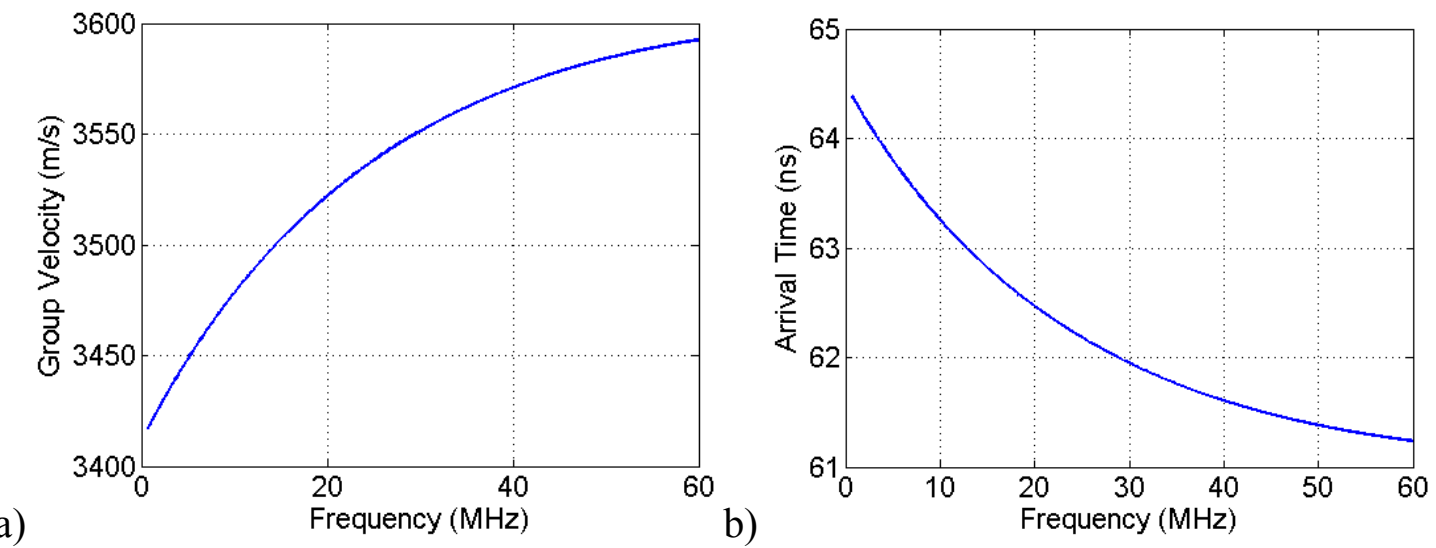

a)

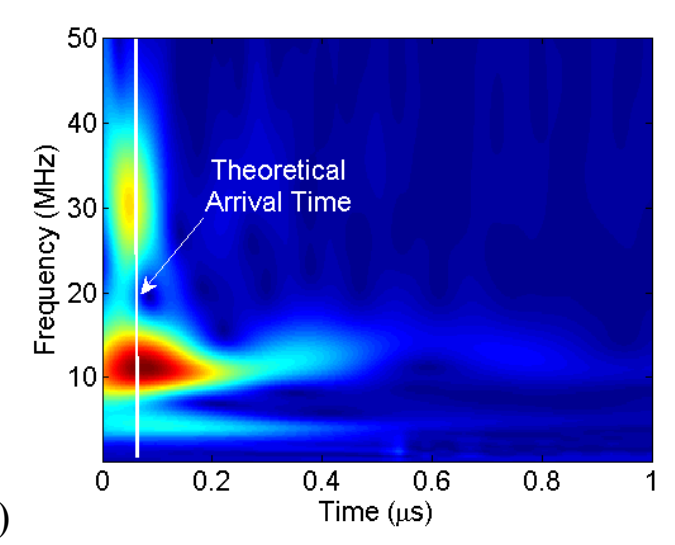

Figure 6.5. Theoretical prediction of the arrival time using the analytical model presented in chapter 5: a) group velocity spectra, b) arrival time as function of frequency, c) comparison of the theory with the experiments.

\subsection{Sensitivity and Optimization}

In this section, several sensitivity measurements are defined and an optimization procedure based on the wave propagation on SAW sensors is described. Several parameters of the wave propagation, such as phase velocity or attenuation can be used to monitor changes on the properties of the fluid surrounding the sensor. Sensitivity can be defined as the ratio of a change of a certain parameter of the propagating wave to the change in the property of the surrounding fluid. Therefore, the sensor sensitivity can be defined as:

$$
S_{\mathrm{v}_{g}}=\frac{\Delta \mathrm{v}_{g} / \mathrm{v}_{g}}{\Delta \rho^{f} / \rho^{f}}, \quad \text { and } \quad S_{\gamma}=\frac{\Delta \gamma / \gamma}{\Delta \rho^{f} / \rho^{f}},
$$

where $\gamma$ is the attenuation of the amplitude of the wave due to radiation of energy to the fluid, $\mathrm{v}_{g}$ is the group velocity and $\rho^{f}$ is the density of the fluid. Using Eqs. (5.24) and (5.25), the sensitivities of Rayleigh and Lamb wave sensor can be analytically calculated.

For the case of Rayleigh wave sensors it was found that the sensitivity for air does not depend on the frequency (Figure 6.6). However, when the external fluid is water, there is a small variation of the sensitivity as a function of the frequency and thickness of the piezoelectric (Figure 6.6). Notice that the sensitivity of the group velocity for a 
change in the density of air or water is very small. Therefore, the group velocity should not be used as a probe for density sensing. On the other hand, sensitivities close to 1.0 are obtained for the attenuation. This means that a ratio $1: 1$ is obtained between the changes in attenuation and density.
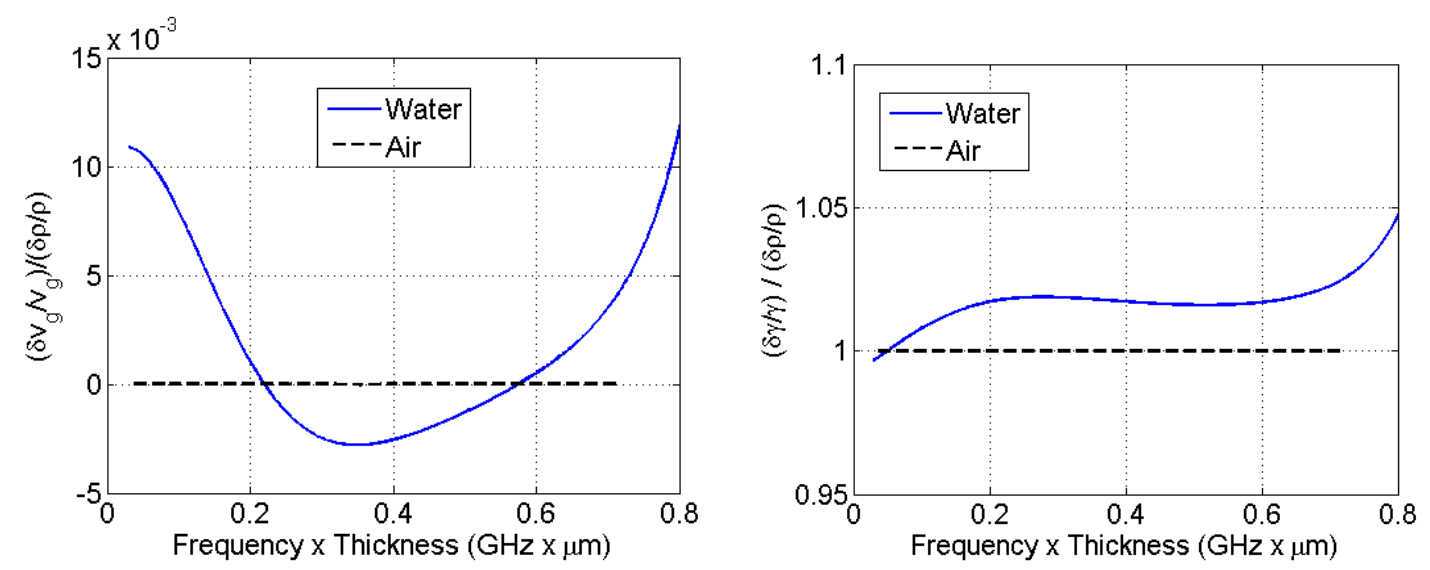

Figure 6.6. Comparison of the sensitivity for a Rayleigh sensor for air and water as surrounding fluids: a) Sensitivity using the group velocity as a probe, b) Sensitivity using attenuation as a probe.

Due to the difference in the shear wave velocities between the AlN layer and the $\mathrm{SiO}_{2}$ substrate there will be a transition from Rayleigh wave to $\mathrm{A}_{0}$ Lamb wave mode when the phase velocity of the Rayleigh wave reaches the shear velocity of the substrate. This transition can be observed in Fig. 6.7. Notice that there is gap in frequency where neither Rayleigh nor $\mathrm{A}_{0}$ Lamb modes exist, however, other modes like $\mathrm{S}_{0}$ may propagate at that frequency band. The sensitivities for $\mathrm{A}_{0}$ and $\mathrm{S}_{0}$ Lamb modes are presented in Fig. 6.8. It can be observed that the sensitivity of the attenuation of the $\mathrm{A}_{0}$ mode increases asymptotically to 1.0 ; while the sensitivity of the $\mathrm{S}_{0}$ mode is very small compared with that of the $\mathrm{A}_{0}$ mode. On the other hand, the sensitivity of the group velocity has comparable values for both modes, however is small when compared with the attenuation sensitivity of the $\mathrm{A}_{0}$ mode.

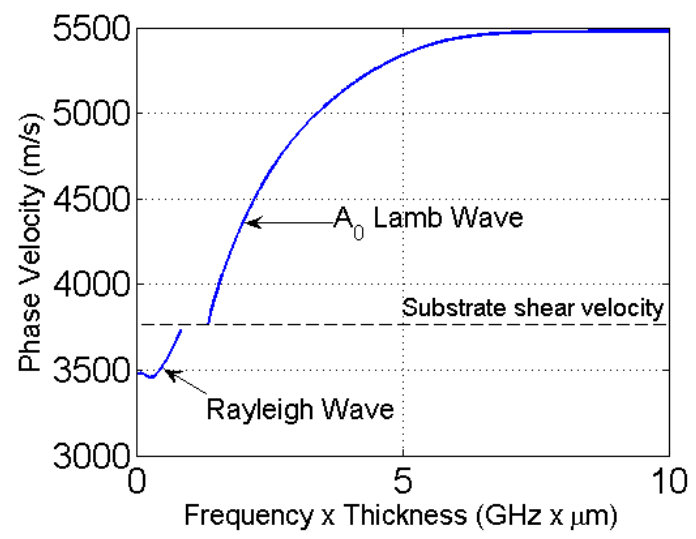

Figure 6.7. Transition form Rayleigh wave to $A_{0}$ Lamb Wave for a sensor composed of an $\mathrm{AlN}$ layer on a $\mathrm{SiO}_{2}$ substrate. 

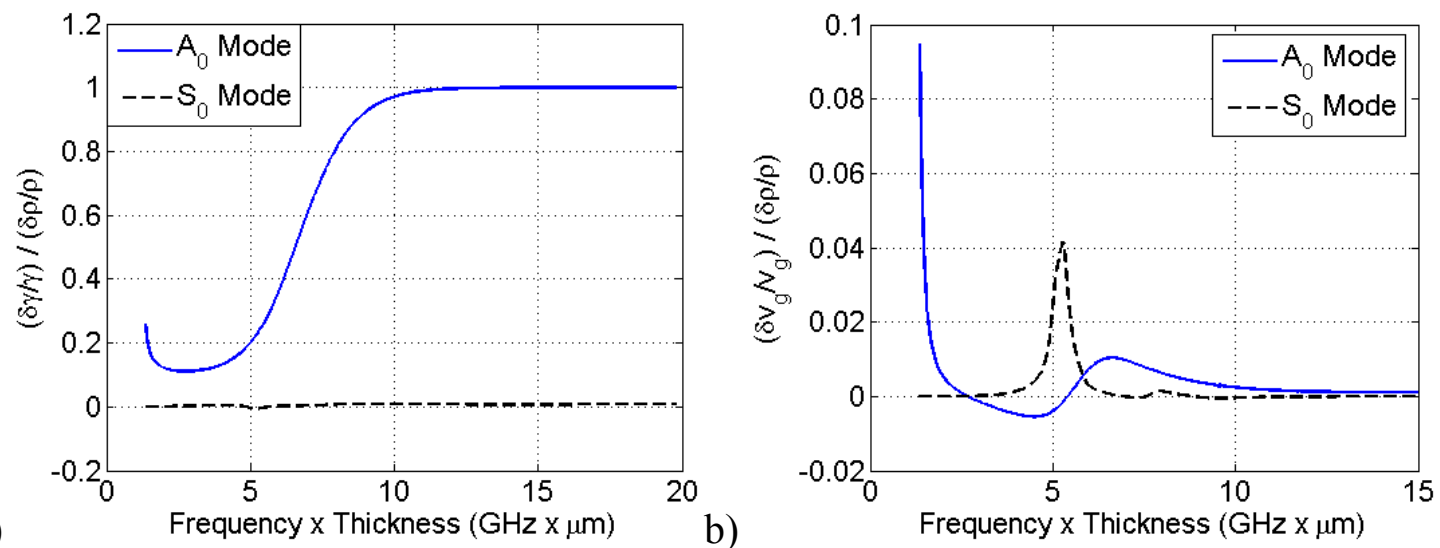

Figure 6.8. Density sensitivity using attenuation (a) and group velocity (b) for a sensor composed of an AlN layer on $\mathrm{SiO}_{2}$ substrate.

The model presented in Chapter 5 can be also used to analyze the wave propagation and sensitivity of Lamb wave sensors. This type of sensor is characterized by having fluid on both sides of the diaphragm. Figure 6.9 shows the dimensions and materials for the sensor considered in this analysis. The sensitivities of the attenuation and group velocity for air and water are shown in Fig. 6.10. It can be observed the sensitivities for the attenuation are similar for the $A_{0}$ and $S_{0}$ for both air and water as surrounding fluids. However, the sensitivity of the group velocity for air is negligible when compared to that of water. In general, the sensitivity of the attenuation has always shown higher values than the sensitivity using velocities as probe.

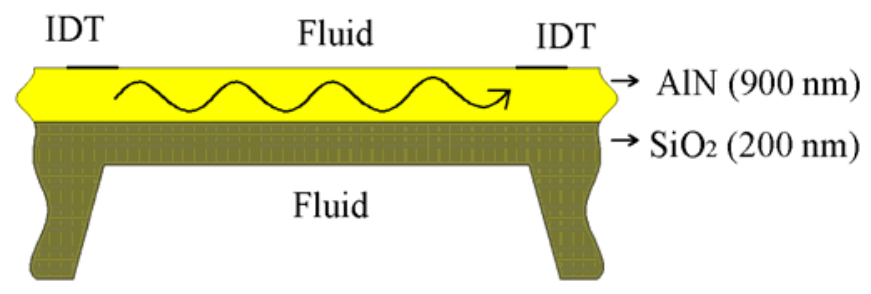

Figure 6.9. Dimensions and materials for a Lamb wave sensor.

a)

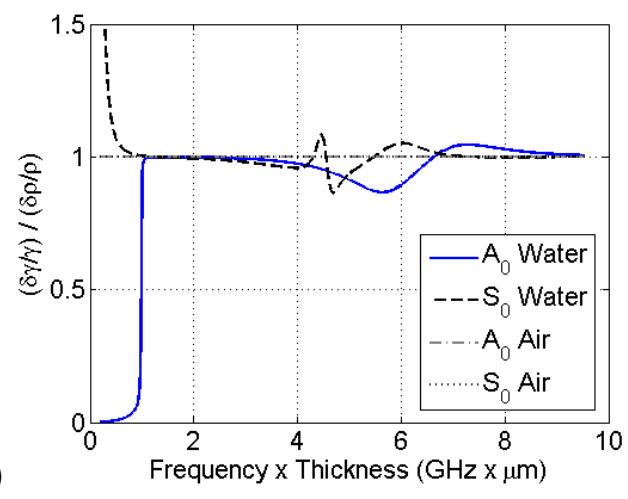

b)

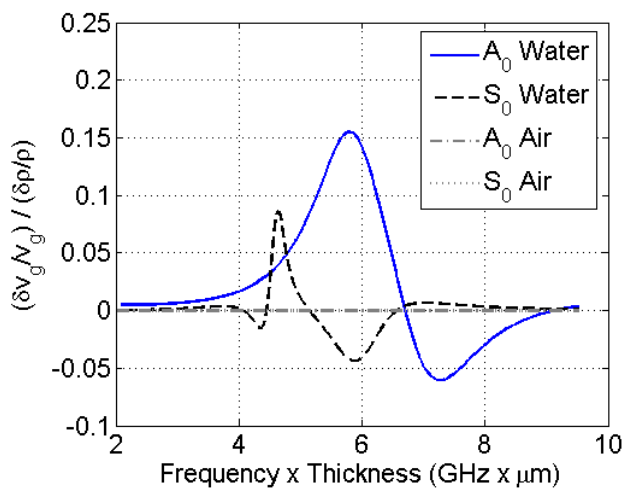

Figure 6.10. Sensitivity of the $A_{0}$ and $S_{0}$ modes for a Lamb wave sensor: a) using the attenuation, b) using the group velocity. 
For Rayleigh and Lamb wave sensors, the wavelength of the excited wave is determined by the width of the IDT fingers. This implies that wave excited will have mainly one wavelength component. From the dispersion equations, the frequency associated with that wavelength can be obtained. Therefore, the sensor can only be operated at a single frequency, which is indirectly determined by the dimensions of the IDTs. For these reasons, it is very important to determine at which frequency the maximum sensitivity is obtained. The analytical solution presented above can be used to obtain the sensitivity as a function of the frequency and wavelength. Therefore, the design of the sensor and the IDTs can be optimized to get the maximum sensitivity. In addition to this, the solution also allows analyzing which of the wave parameters $\left(\gamma, \mathrm{v}_{p}\right.$ or $\mathrm{v}_{g}$ ) is more suitable to use as a probe for the sensor for a given application. The optimization procedure is illustrated in the flow chart presented in Fig. 6.11.

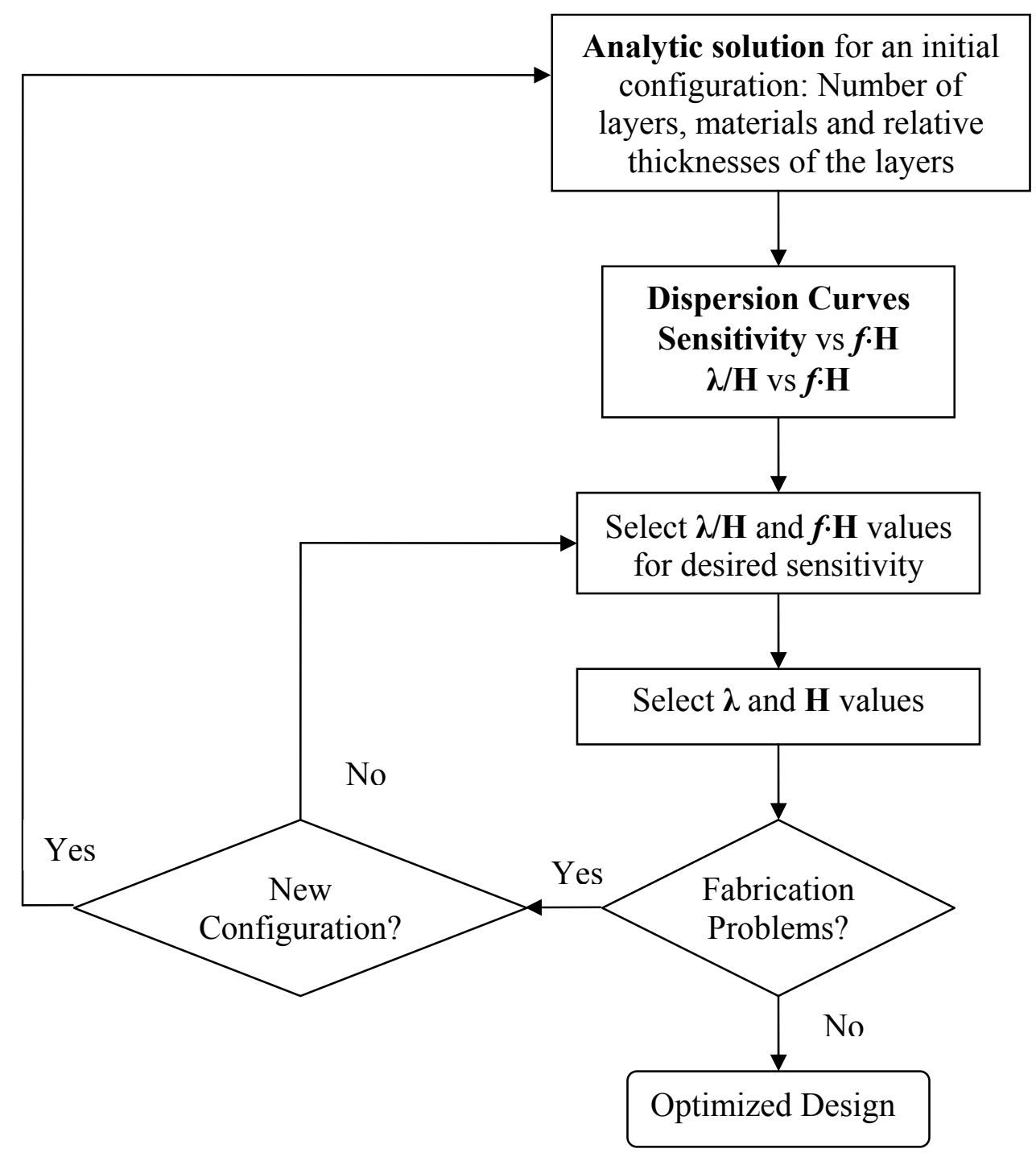

Figure 6.11. Optimization procedure for Lamb-wave sensors using the analytic solution presented here. 
The analytical model presented in Chapter 5 can be used to get all the parameters required optimize the sensor. The optimization procedure will be illustrated for a Lamb wave sensor using the sensitivity of the group velocity for $\mathrm{A}_{0}$ mode. It can be observed that there is a value of Frequency $\times$ Thickness $(f \cdot \mathrm{H}=5.8 \mathrm{GHz} \cdot \mu \mathrm{m})$ for which the sensitivity is maximal (Fig. 6.12a). From the dispersion curves, a relation between the wavelength and the frequency can be obtained for this mode (Fig. 6.12b). Therefore, the value of the ratio $\lambda / \mathrm{H}$ for maximum sensitivity is 0.8 . In general, the fabrication process imposes restrictions on the minimum width of the IDT fingers and maximum thickness that can be achieved by photolithography and sputtering. In this example the IDT's finger width $2 \mu \mathrm{m}$ was selected; therefore a total thickness of the diaphragm should be $2.5 \mu \mathrm{m}$ to obtain the maximum sensitivity.
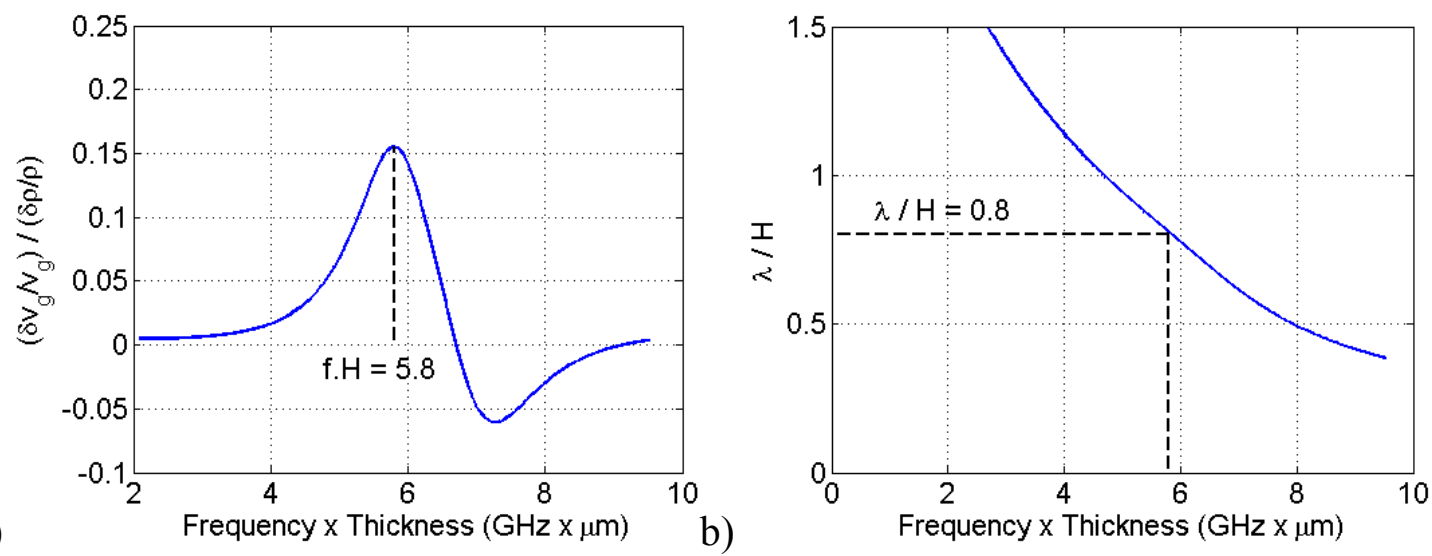

Figure 6.12. Procedure to design parameters for maximum sensitivity. a) $f \cdot \mathrm{H}$ values and b) $\lambda / \mathrm{H}$.

\subsection{Conclusions}

In this chapter, the modeling of Rayleigh and Lamb wave sensors was presented. Several important parameters of the sensor such as coupling coefficient and sensitivity were determined analytically. It was observed that the sensitivity of the attenuation has higher values than that of the wave velocities when air or water were considered as surrounding fluids. Additionally, the sensitivity of the wave velocity for air was negligible when compared to that for water. A procedure to optimize the dimension of the components of the sensor based on the dispersion curves and the sensitivity was proposed and illustrated. 


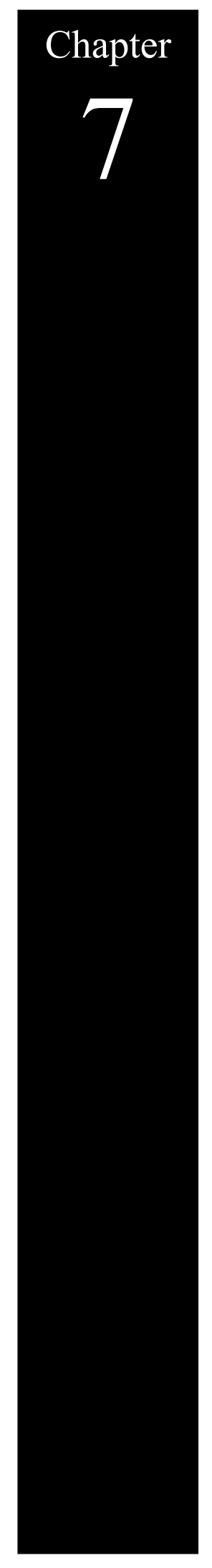

Conclusions and Future Work 
This dissertation was focused on the analytical and numerical modeling of ultrasound transducer and acoustic sensors. Single-element ultrasound transducers were analyzed numerically and analytically in Chapters 3 and 5, respectively. In these chapters, the wave propagation and resonance frequencies were obtained numerically and analytically. It was found that when free-boundary conditions are assumed for the piezoelectric plate, the resonance frequencies at the ZGV points are more significant than those at the cut-off frequencies. However, this relation changes when a fluid or solid is bounded to the plate. It was also observed that resonance frequencies correspond to the frequencies at which the absolute values of wave number are minima. Another important finding related to the effect of the presence of a fluid or solid around the plate is that some modes do not longer have cut-off frequencies, but instead a transition frequency from highly attenuated to a propagating state was observed. The effect of inversion layer was found to slightly change the values of the first and second resonance frequencies, which are very important for the design of harmonic imaging transducers.

On the other hand, 1D array ultrasound transducers are modeled as periodic piezoelectric plates with finite cross-section. A 2D semi-analytic finite-element formulation combined with periodic boundary conditions was developed to analyze the dispersion behavior of these structures. Dispersion curves, group velocity spectra and mode shapes were obtained using the method presented here. The effect of geometrical parameters such as aspect ratio, subdicing width and depth, matching layers and inversion layers were analyzed using this model. In general, the lower aspect ratios, as well as lower subdicing depth, tend to increase the cut-off frequency of all modes. Inversion layer was shown to have discrete effect of the dispersion relation for this type of structures. Matching layers caused some resonance modes to cluster over a certain frequency range. The method presented here can be used to analyze complicated geometries for 1D array ultrasound transducers.

Finally, it was found that the analytical model presented for single-element ultrasound transducer could be also used to model Rayleigh and Lamb wave sensors. This analytical formulation allows the calculation of important parameters of the transducers like sensitivity. As part of the validation of the model, the wave velocity was obtained experimentally and compared with the analytical prediction; a good agreement between them was obtained. It was observed that the sensitivity of the attenuation has higher values than that of the wave velocities when air or water were considered as surrounding fluids.

In the future, several studies can continue the developments presented in this dissertation. As mentioned before, this dissertation was focused in the analytical and numerical modeling of piezoelectric devices. However, it would be desirable to evaluate the improvement on the efficiency of the transducer and quality of the ultrasound images obtained using transducers designed with the methodologies presented in this study. In the same way, acoustic sensor designed according the optimization procedure presented here can be fabricated and tested for several environments and applications. 


\section{Appendix A: Mass and Stiffness Matrices for 1D SAFE}

The matrices shown in Eq. (3.3) are defined as,

$$
\mathbf{B}_{1}=\left[\begin{array}{cccc}
\mathbf{N} & \cdot & \cdot & \cdot \\
\cdot & \cdot & \cdot & \cdot \\
\cdot & \cdot & \cdot & \cdot \\
\cdot & \cdot & \cdot & \cdot \\
\cdot & \cdot & \mathbf{N} & \cdot \\
\cdot & \mathbf{N} & \cdot & \cdot \\
\cdot & \cdot & \cdot & -\mathbf{N} \\
\cdot & \cdot & \cdot & \cdot \\
\cdot & \cdot & \cdot & \cdot
\end{array}\right] \text {, and } \mathbf{B}_{2}=\left[\begin{array}{cccc}
\cdot & \cdot & \cdot & \cdot \\
\cdot & \cdot & \cdot & \cdot \\
\cdot & \cdot & \mathbf{N}_{, z} & \cdot \\
\cdot & \mathbf{N}_{, z} & \cdot & \cdot \\
\mathbf{N}_{, z} & \cdot & \cdot & \cdot \\
\cdot & \cdot & \cdot & \cdot \\
\cdot & \cdot & \cdot & \cdot \\
\cdot & \cdot & \cdot & \cdot \\
\cdot & \cdot & \cdot & -\mathbf{N}_{, z}
\end{array}\right] \text {, }
$$

where

$$
\mathbf{N}=\left[\begin{array}{cccccccccccc}
N_{1} & \cdot & \cdot & \cdot & N_{2} & \cdot & \cdot & \cdot & N_{3} & \cdot & \cdot & \cdot \\
\cdot & N_{1} & \cdot & \cdot & \cdot & N_{2} & \cdot & \cdot & \cdot & N_{3} & \cdot & \cdot \\
\cdot & \cdot & N_{1} & \cdot & \cdot & \cdot & N_{2} & \cdot & \cdot & \cdot & N_{3} & \cdot \\
\cdot & \cdot & \cdot & N_{1} & \cdot & \cdot & \cdot & N_{2} & \cdot & \cdot & \cdot & N_{3}
\end{array}\right] .
$$

$N_{1}, N_{2}$ and $N_{3}$ are quadratic interpolation functions with three equally spaced nodal surfaces given as,

$$
N_{1}=-\frac{1}{2} \varsigma(1-\varsigma), N_{2}=1-\varsigma^{2}, \text { and } N_{3}=\frac{1}{2} \varsigma(1+\varsigma), \text { where }-1 \leq \varsigma \leq 1
$$

Equation (3.5) can be expressed in terms of Eq. (3.3) as:

$$
\begin{aligned}
& K E=\frac{1}{2} \int \dot{\mathbf{v}}^{e T} \mathbf{m}^{e} \dot{\mathbf{v}}^{e} d x, \\
& H=\frac{1}{2} \int\left(\mathbf{v}_{, x}^{e T} \mathbf{k}_{11} \mathbf{v}_{, x}^{e}+\mathbf{v}_{, x}^{e T} \mathbf{k}_{12} \mathbf{v}^{e}+\mathbf{v}^{e T} \mathbf{k}_{21} \mathbf{v}_{, x}^{e}+\mathbf{v}^{e T} \mathbf{k}_{22} \mathbf{v}^{e}\right) d x, \\
& W=\frac{1}{2} \int \mathbf{v}^{e T} \mathbf{f}^{e} d x,
\end{aligned}
$$

where,

$$
\mathbf{m}^{e}=\int \mathbf{N}^{T} \mathbf{\rho} \mathbf{N} d z, \quad \mathbf{k}_{i j}=\int \mathbf{B}_{i}^{T} \mathbf{C B}{ }_{j} d z, \quad \text { and } \mathbf{f}^{e}=\int \mathbf{N}^{T} \mathbf{f} d z, \quad \text { for } i, j=1,2,3 .
$$

Eq. (3.4) can be rewritten using Eq. (A.2) as

$$
\delta \int_{t_{0}}^{t_{1}}\left[\frac{1}{2} \int\left(\dot{\mathbf{v}}^{e T} \mathbf{m}^{e} \dot{\mathbf{v}}^{e}-\mathbf{v}^{e T}{ }_{, x} \mathbf{k}_{11}^{e} \mathbf{v}_{, x}^{e}-\mathbf{v}_{, x}^{e T} \mathbf{k}_{12}^{e} \mathbf{v}^{e}-\mathbf{v}^{e T} \mathbf{k}_{21}^{e} \mathbf{v}_{, x}^{e}-\mathbf{v}^{e T} \mathbf{k}_{22}^{e} \mathbf{v}^{e}-\mathbf{v}^{e T} \mathbf{f}^{e}\right) d x\right] d t=0,
$$

Assembling the global matrices according to the connectivity of the elements, Eq. (A.3) can be written in terms of the global matrices as:

$\delta \int_{t_{0}}^{t_{1}}\left[\frac{1}{2} \int\left(\dot{\mathbf{V}}^{T} \mathbf{M} \dot{\mathbf{V}}-\mathbf{V}_{, x}^{T} \mathbf{K}_{11} \mathbf{V}_{, x}-\mathbf{V}_{, x}^{T} \mathbf{K}_{12} \mathbf{V}-\mathbf{V}^{T} \mathbf{K}_{21} \mathbf{V}_{, x}-\mathbf{V}^{T} \mathbf{K}_{22} \mathbf{V}-\mathbf{V}^{T} \mathbf{F}\right) d x\right] d t=0$

where, 


$$
\mathbf{M}=\bigcup \mathbf{m}^{e}, \quad \mathbf{K}_{i j}=\bigcup \mathbf{k}_{i j}^{e}, \mathbf{F}=\bigcup \mathbf{f}^{e} \text {, and } \mathbf{V}=\bigcup \mathbf{v}^{e} .
$$

Carrying out the variations in Eq. (A4), the global system of equation of motion is obtained

$$
\mathbf{M V}+\mathbf{K}_{1} \mathbf{V}+\mathbf{K}_{2} \mathbf{V}_{, X}-\mathbf{K}_{3} \mathbf{V}_{, X X}=\mathbf{F}
$$

where $\mathbf{K}_{2}=\mathbf{K}_{21}-\mathbf{K}_{12}$. 


\section{Appendix B: Mass and Stiffness Matrices for 2D SAFE}

The terms of the Eq. (4) can be written in terms of $\mathbf{v}$ y $\mathbf{q}$ as follows:

$$
\begin{gathered}
K E=\frac{1}{2} \iiint_{\mathcal{U}} \dot{\mathbf{v}}^{T} \boldsymbol{\rho} \dot{\mathbf{v}} d v, H=\frac{1}{2} \iiint_{\mathcal{U}} \mathbf{q}^{T} \mathbf{C q} d v, \text { and } W=\iint_{S} \mathbf{v}^{T} \mathbf{f} d S, \\
\mathbf{C}=\left[\begin{array}{cc}
\mathbf{c} & -\mathbf{e}^{T} \\
-\mathbf{e} & -\mathbf{\varepsilon}
\end{array}\right] \text { and } \boldsymbol{\rho}=\left[\begin{array}{cccc}
\rho & & \\
& \rho & & \\
& \rho & \\
& & 0
\end{array}\right] .
\end{gathered}
$$

where,

$$
\mathbf{q}=\left(\mathbf{L}_{x}+\mathbf{L}_{y z}\right) \mathbf{N}^{e} \mathbf{v}^{e}=\mathbf{B}_{1} \mathbf{v}_{, x}^{e}+\mathbf{B}_{2} \mathbf{v}^{e}
$$

Where:

$$
\begin{aligned}
\mathbf{L}_{x} & =\left[\begin{array}{cccc}
\partial_{x} & \cdot & \cdot & \cdot \\
\cdot & \cdot & \cdot & \cdot \\
\cdot & \cdot & \cdot & \cdot \\
\cdot & \cdot & \cdot & \cdot \\
\cdot & \cdot & \partial_{x} & \cdot \\
\cdot & \partial_{x} & \cdot & \cdot \\
\cdot & \cdot & \cdot & -\partial_{x} \\
\cdot & \cdot & \cdot & \cdot \\
\cdot & \cdot & \cdot & \cdot
\end{array}\right], \mathbf{L}_{y z}=\left[\begin{array}{cccc}
\cdot & \cdot & \cdot & \cdot \\
\cdot & \partial_{y} & \cdot & \cdot \\
\cdot & \cdot & \partial_{z} & \cdot \\
\cdot & \partial_{z} & \partial_{y} & \cdot \\
\partial_{z} & \cdot & \cdot & \cdot \\
\partial_{y} & \cdot & \cdot & \cdot \\
\cdot & \cdot & \cdot & \cdot \\
\cdot & \cdot & \cdot & -\partial_{y} \\
\cdot & \cdot & \cdot & -\partial_{z}
\end{array}\right], \\
\mathbf{N}^{e} & =\left[\begin{array}{cccc}
\mathbf{N} & \cdot & \cdot & \cdot \\
\cdot & \mathbf{N} & \cdot & \cdot \\
\cdot & \cdot & \mathbf{N} & \cdot \\
\cdot & \cdot & \cdot & \mathbf{N}
\end{array}\right] .
\end{aligned}
$$

Here, $\mathbf{N}=\left[N_{1} N_{2} N_{3} N_{4}\right]$ are the isoparametric interpolation functions for a four-node $2 \mathrm{D}$ element. Eq. (B1) can be expressed in terms of Eq. (B2) as:

$$
\begin{aligned}
& K E=\frac{1}{2} \iint \dot{\mathbf{v}}^{e T} \mathbf{m}^{e} \dot{\mathbf{v}}^{e} d x d y \\
& H=\frac{1}{2} \iint\left(\mathbf{v}_{, x}^{e T} \mathbf{k}_{11} \mathbf{v}_{, x}^{e}+\mathbf{v}_{, x}^{e T} \mathbf{k}_{12} \mathbf{v}^{e}+\mathbf{v}^{e T} \mathbf{k}_{21} \mathbf{v}_{, x}^{e}+\mathbf{v}^{e T} \mathbf{k}_{22} \mathbf{v}^{e}\right) d x d y \\
& W=\frac{1}{2} \int \mathbf{v}^{e T} \mathbf{f}^{e} d x,
\end{aligned}
$$

and,

$$
\mathbf{m}^{e}=\iint \mathbf{N}^{T} \mathbf{\rho} \mathbf{N} d y d z, \quad \mathbf{k}_{i j}^{e}=\iint \mathbf{B}_{i}{ }^{T} \overline{\mathbf{C}} \mathbf{B}_{j} d y d z \quad \text { for } i, j=1,2 \text { and }
$$


The Hamilton's principle in terms of single element displacements and velocities can be written as

$$
\delta \int_{t_{0}}^{t_{1}}\left[\frac{1}{2} \int\left(\dot{\mathbf{v}}^{e T} \mathbf{m}^{e} \dot{\mathbf{v}}^{e}-\mathbf{v}^{e T}{ }_{, x} \mathbf{k}_{11}^{e} \mathbf{v}_{, x}^{e}-\mathbf{v}^{e T}{ }_{, x} \mathbf{k}_{12}^{e} \mathbf{v}^{e}-\mathbf{v}^{e T} \mathbf{k}_{21}^{e} \mathbf{v}^{e}{ }_{, x}-\mathbf{v}^{e T} \mathbf{k}_{22}^{e} \mathbf{v}^{e}-\mathbf{v}^{e T} \mathbf{f}^{e}\right) d x\right] d t=0,
$$

An equivalent equation to (A4) for global coordinates can be assembled from the element matrices according to their connectivity

$$
\delta \int_{t_{0}}^{t_{1}}\left[\frac{1}{2} \int\left(\dot{\mathbf{V}}^{T} \mathbf{M} \dot{\mathbf{V}}-\mathbf{V}_{, x}^{T} \mathbf{K}_{11} \mathbf{V}_{, x}-\mathbf{V}^{T}{ }_{, x} \mathbf{K}_{12} \mathbf{V}-\mathbf{V}^{T} \mathbf{K}_{21} \mathbf{V}_{, x}-\mathbf{V}^{T} \mathbf{K}_{22} \mathbf{V}-\mathbf{V}^{T} \mathbf{F}\right) d x\right] d t=0 .
$$

Carrying out the variation of Eq. (B5) the following system of differential equations is obtained

$$
\mathbf{K}_{1} \mathbf{V}_{, X X}-\mathbf{K}_{2} \mathbf{V}_{, X}-\mathbf{K}_{3} \mathbf{V}-\mathbf{M} \ddot{\mathbf{V}}=\mathbf{F}
$$

where

$\mathbf{K}_{1}=\mathbf{K}_{11}, \mathbf{K}_{2}=\mathbf{K}_{21}-\mathbf{K}_{12}, \mathbf{K}_{3}=\mathbf{K}_{22}$.

\section{Appendix C: Transfer Matrix}

A convenient procedure to handle these boundary conditions is called the Transfer Matrix method (Mal, 1988). For each layer of the plate, the displacement and stresses on each side of the layer can be linearly related through the transfer matrix. The displacements and stresses (Eqs. 5.2 and 5.3) at the top of each layer can be expressed as:

$$
\left\{\begin{array}{c}
\hat{u}_{1} \\
\hat{u}_{3} \\
\hat{\phi} \\
\hat{T}_{33}^{*} \\
\hat{T}_{13}^{*} \\
\hat{D}_{3}^{*}
\end{array}\right\}^{m+}=\left[\begin{array}{cccccc}
U_{1} & U_{2} & U_{3} & U_{4} & U_{5} & U_{6} \\
W_{1} & W_{2} & W_{3} & W_{4} & W_{5} & W_{6} \\
\Phi_{1} & \Phi_{2} & \Phi_{3} & \Phi_{4} & \Phi_{5} & \Phi_{6} \\
D_{11} & D_{12} & D_{13} & D_{14} & D_{15} & D_{16} \\
D_{21} & D_{22} & D_{23} & D_{24} & D_{25} & D_{26} \\
D_{31} & D_{32} & D_{33} & D_{34} & D_{35} & D_{36}
\end{array}\right]^{m}\left\{\begin{array}{l}
\beta_{1} e^{i \zeta \alpha_{1} h / 2} \\
\beta_{2} e^{i \zeta \alpha_{2} h / 2} \\
\beta_{3} e^{i \zeta \alpha_{3} h / 2} \\
\beta_{4} e^{i \zeta \alpha_{4} h / 2} \\
\beta_{5} e^{i \zeta \alpha_{5} h / 2} \\
\beta_{6} e^{i \zeta \alpha_{6} h / 2}
\end{array}\right\}^{m+}
$$

The superscript $m+$ corresponds to the layer $m$ and top surface, $h$ is the thickness of the layer and $\hat{T}_{33}^{*}=\hat{T}_{33} / i \zeta$. Equation $(\mathrm{C} 1)$ can be rewritten in an abbreviated for as:

$$
P^{m+}=X^{m} D^{m+} \beta^{m},
$$

where $D^{m+}$ is a diagonal $6 \times 6$ matrix with $e^{i \zeta \alpha_{q} h / 2}(q=1, \ldots, 6)$ as elements of the diagonal. For the bottom surface, an equivalent equation can be written as:

$$
P^{m-}=X^{m} D^{m-} \beta^{m} \text {. }
$$

The vector $P^{m-}$ represents the displacements and stresses of the bottom surface and the matrix $D^{m-}$ is diagonal with $e^{-i \zeta \alpha_{q} h / 2}(q=1, \ldots, 6)$ as its elements. Note that $\beta^{m}$ is a common vector for Eqs. (C2) and (C3). Therefore, they can be combined into a single relation as shown:

$$
P^{m+}=X^{m} D^{m+}\left(D^{m-}\right)^{-1}\left(X^{m}\right)^{-1} P^{m-}=A^{m} P^{m-},
$$


where the matrix $A^{m}$ is the transfer matrix of the layer $m$. This matrix linearly relates the displacement and stresses of top and bottom surfaces for the layer $m$. This representation of the solution is convenient to handle the continuity conditions between layers, which can be simply expressed by $P^{m^{+}}=P^{(m+1)-}$ for the layer $m$ and $(m+1)$. For an $N$-layered plate the following relation is obtained:

$$
P^{+}=A^{N} A^{N-1} \ldots A^{2} A^{1} P=A P^{-},
$$

where $A$ is global transfer matrix, $P^{+}$and $P^{-}$are the displacements and stresses at the top and bottom of the entire laminated plate.

For the dielectric half-space solid the displacements and stresses can be expressed in a form equivalent to Eq. (C1). However, since radiation of energy from the plate to the half-space solid is expected, two partial waves which have increasing amplitude with depth are the only ones present in the half-space solid; therefore, the amplitude $\beta$ of the other two partial waves must be equal to zero

$$
\left\{\begin{array}{c}
\hat{u}_{1} \\
\hat{u}_{3} \\
\hat{\phi} \\
\hat{T}_{33}^{*} \\
\hat{T}_{13}^{*} \\
\hat{D}_{3}^{*}
\end{array}\right\}=\left[\begin{array}{ccc}
U_{1} & U_{2} & 0 \\
W_{1} & W_{2} & 0 \\
0 & 0 & 1 \\
D_{11} & D_{12} & 0 \\
D_{21} & D_{22} & 0 \\
0 & 0 & i \varepsilon^{s}
\end{array}\right]^{h s}\left\{\begin{array}{c}
\beta_{1} e^{i \zeta \alpha_{1}\left(x_{3}+H / 2\right)} \\
\beta_{2} e^{i \zeta \alpha_{2}\left(x_{3}+H / 2\right)} \\
\Phi_{s} e^{\zeta\left(x_{3}+H / 2\right)}
\end{array}\right\} .
$$

Equation (C6) is valid for $\mathrm{x} 3<-\mathrm{H} / 2$, where $\mathrm{H}$ is total thickness of the laminated plate. 


\section{References}

Abdollahi, A., Jiang, Z., Arabshahi, S. A., 2007. Evaluation on mass sensitivity of SAW sensors for different piezoelectric materials using finite-element analysis. IEEE Transactions on Ultrasonics, Ferroelectrics, and Frequency Control 54, 24462455.

Achenbach, J.D., Keshava, S.P., 1967. Free waves in plate supported by a semi-infinite continuum. Journal of Applied Mechanics 34, 397-404.

Akiyama, I., Ohya A., Saito, S., 2005. Speckle noise reduction by superposing many higher harmonic images. Japanese Journal of Applied Physics 44(6B), 46314634.

Auld, B. A., 1990. Acoustic Fields and Waves in Solids, vol. II, 2nd ed. Malabar, FL: Krieger.

Averkiou, M.A., 2000. Tissue harmonic imaging. IEEE Ultrasonic Symposium Procedures, 1561-1566.

Bai, H., Taciroglu, E., Dong, S.B., Shah, A.H., 2004. Elastodynamic green's functions for a laminated piezoelectric cylinder. International Journal of Solids and Structures 41, 6335-6350.

Ballandras, S., Wilm, M., Edoa, P.F., Soufyane, A., Laude, V., 2003. Finite-element analysis of periodic piezoelectric trasducers. Journal of Applied Physics 93(1), 702-711.

Ballandras, S., Wilm, M., Daniau, W., Reinhardt, V., Laude, V., Armati, R., 2005. Periodic finite element/boundary element modeling of capacitive micromachined ultrasonic transducers. Journal of Applied Physics 97(3) 034901.

Ballentine, D. S., White, R. M., Martin, S. J., Ricco, A. J., Frye, G. C., Zellers, E. T., Wohltjen, H., 1997. Acoustic Wave Sensors: Theory, Design and PhysicoChemical Applications, vol. Academic Press. San Diego.

Benetti, M., Cannata, D., Di Pietrantonio, F., Verona, E., 2005. Growth of a AlN piezoelectric film on diamond for high-frequency surface acoustic wave devices. IEEE Transaction on Ultrasonics Ferroelectrics and Frequency Control 52(10), 1806-1811.

Bouakaz, A., Frigstad, S., Ten Cate, F.J., de Jong, N., 2002. Super harmonic imaging: A new imaging technique for improved contrast detection. Ultrasound in Medicine \& Biology 28(1), 59-68.

Caliendo, C., Verardi, C., Verona, E., D’Amico, A., Di Natale, C., Saggio, G., Serafini, M., Paolesse, R., Hua, S., 2008. Advances in SAW-based gas sensors. Smart Materials and Structures 6, 689-699.

Caronti, A., Savoia, A., Caliano, G., and Pappalardo, M., 2005. Acoustic coupling in capacitive microfabricated ultrasonic transducers: modeling and experiments. IEEE Transactions On Ultrasonics Ferroelectrics And Frequency Control 52(12), 2220-2234. 
Chan, H.L.W., Unsworth, J., 1987. Effect of ceramic anisotropy on the properties of ceramic/polymer piezoelectric composites. Proceedings of the 1987 IEEE Ultrasonics Symposium, p. 651.

Clorennec, D., Prada C., Royer, D., 2007. Local and non-contact measurements of bulk acoustic wave velocities in thin isotropic plates and shells using zero group velocity Lamb modes Journal of Applied Physics 101(3), 034908.

Cortes, D.H., Datta, S.K., Mukdadi, O.M., 2008a. Dispersion behavior of elastic guided wave propagation in layered piezoelectric infinite plates with inversion layer. International Journal of Solids and Structures 45, 5088-5102.

Cortes, D.H., Korakakis, D., Kabulski, A., Mukdadi, O. M., 2008b. Dispersion analysis of high-frequency nanoscale surface acoustic wave sensors for detecting biological and chemical species in harsh environment. ASME International Mechanical Congress and Exposition, Boston, MA.

Dickherber, A., Corso, C. D., hunt, W. D., 2009. Optimization and characterization of a $\mathrm{ZnO}$ biosensor array. Sensors and Actuators, In Press.

Duhamel, R., Robert, L., Jia, H., Li, F., Lardet-Vieudrin, F., Manceau, J. F., Bastien, F., Sensitivity of a Lamb wave sensor with 2 um AlN membrane. Ultrasonics 44, e893-e897.

El Hakiki, M., Elmazria, O., Assouar, M.B., Mortet, V., Talbi, A., Sarry, F., 2004. High SAW velocity and high electromechanical coupling coefficient with the new three layered structure: $\mathrm{ZnO} / \mathrm{AlN} /$ Diamond. Proceedings of the 2004 IEEE Ultrasonics Symposium, p. 195.

Gibson, A., Popovics, J.S., 2005. Lamb wave basis for impact-echo method analysis. Journal of Engineering Mechanics 131(4), 438 - 443.

Goldberg, R.L., Jurgens, M.J., Mills, D.M., Henriquez, C.S., Vaughan, D., and Smith, S.W., 1997. Modeling of piezoelectric multilayer ceramics using finite element analysis. IEEE Transactions On Ultrasonics Ferroelectrics And Frequency Control 44(6), 1204-1214.

Greenwood, M.S., Adamson, J.D., Bond, L.J., 2006. Measurement of viscosity-density product using multiple reflections of ultrasonic shear horizontal waves. Ultrasonics 44, 1031-1036.

Gronewold, T. M. A., 2007. Surface acoustic wave sensors in the bioanalytical field: Recent trends and challenges. Analytica Chimica Acta 603, 119-128.

Holland, S.D., Chimenti, D.E., 2004. High contrast air-coupled acoustic imaging with zero group velocity Lamb modes. Ultrasonics 42, $957-960$.

Hossack J.A., Hayward G., 1991. Finite-element analysis of 1-3 composite transducers. IEEE Transaction on Ultrasonics Ferroelectrics and Frequency Control 38(6), 618-629.

Hoummady, M., Campitelli, A., Wlodarski, W., 1997. Acoustic wave sensors: Design, sensing mechanisms and applications. Smart Materials and Structures 6, 647-657.

Huang, C., Marmarelis, V.Z., Zhou, Q., Shung, K.K., 2005. An analytical model of multilayer ultrasonic transducers with an inversion layer. IEEE Transaction on Ultrasonics Ferroelectrics and Frequency Control 53(3), 469-479.

Huang, J,H., Yu H.I., 2000. Dynamic electromechanical response of piezoelectric plates as sensor and actuators. Materials Letters 46, 70-80. 
Huang, M.H., Shen, D., Chow, L.M., Yang, M., 2001. Correlations of the impedance parameters and conductivity and permittivity of liquid and gel phases in a series of piezoelectric quartz crystal sensor. Sensors and Actuators B 72, 21-27.

Humphrey, V.F., 2000. Nonlinear propagation in ultrasonic fields: Measurements, modeling and harmonic imaging. Ultrasonics 38,267-272.

Kallard, T., 1971. Acoustic surface wave and acousto-optic devices. Optosonic press, New York, NY.

Khutoryansky, N.M., Sosa, H., 1995. Dynamic representation formulas and fundamental solutions for piezoelectricity. International Journal of Solids and Structures 32, 3307-3325.

Kino, G., 1987. Acoustic waves: devices, imaging and analog signal processing. PrenticeHall, New Jersey.

Kondoh, J., Shiokawa, S., 1995. Shear surface acoustic wave liquid sensor based on acoustoelectric interaction. Electronics and Communications in Japan 78, 101112.

Kornbluth, M., Liang, D.H., Paloma, A., Schnittger, I., 1998. Native harmonic imaging improves endocardial border definition and visualization of cardiac structures. Journal of American Society of Echocardiography 11, 693-701.

Lange, K., Rapp, B.E., Rapp, M., 2008. Surface acoustic wave biosensors: a review. Anal. Bioanal. Chem. 391, 1509-1519.

Lec, R., Vetelino, J.F., Clark, P., Roy, A., Turner, J., 1998. Prototype microwave acoustic fluid sensors. Proceedings of the 1988 IEEE Ultrasonics Symposium, p. 543.

Lee Y.C., Kuo, S.H., 2006. Leaky lamb wave of a piezoelectric plate subjected to conductive fluid loading: Theoretical analysis and numerical calculation. Journal of Applied Physics 100, 073519.

Leedom, D.A., Krimholtz, R., Matthaei, G.L., 1978. Equivalent circuits for transducers having arbitrary even- or odd-symmetry piezoelectric excitation. IEEE Transaction on Sonics and Ultrasonics 25, 115-125.

Lerch, R., 1990. Simulation of piezoelectric devices by two- and three-dimensional finite elements. IEEE Transaction on Ultrasonics Ferroelectrics and Frequency Control 37(2), 233-247.

Lindner, G., 2008. Sensors and actuators based on surface acoustic waves propagating along solid-liquid interfaces. Journal of Physics D: Applied Physics 41, 123002.

Mal, A.K., 1988. Wave propagation in layered composite laminates under period surface loads. Wave Motion 10, 257-266.

Matthews, H., 1977. Surface wave filters: Design, construction and use. John Wiley \& Sons, New York.

McKeighen, R., 1998. Design guidelines for medical ultrasonic arrays. SPIE International Symposium on Medical Imaging, 2-18.

McKeighen, R., 2001. Finite element simulation and modeling of 2-D arrays for 3-D ultrasonic imaging. IEEE Transaction on Ultrasonics Ferroelectrics and Frequency Control 48(5), 1395-1405.

Mills, D.M., Smith S.W., 2002a. Multi-layered PZT/Polymer composites to increase signal-to-noise ratio and resolution for medical ultrasound transducers Part II: Thick film technology. IEEE Transaction on Ultrasonics Ferroelectrics and Frequency Control 49(7), 1005-1014. 
Mills, D.M., Smith S.W., 2002b. Finite element comparison of single crystal vs. multilayer composite arrays for medical ultrasound. IEEE Transaction on Ultrasonics Ferroelectrics and Frequency Control 49(7), 1015-1020.

Miyawaza, S., 1979. Ferroelectric domain inversion in Ti-diffused LiNbO3 optical waveguide. Journal of Applied Physics 50, 4599-4603.

Moll, N., Pascal, E., Dinh, D.H., Lachaud, J.L., Vellutini, L., Pillot, J.P., Rebiere, D., Moynet, D., Pistre, J., Mossalayi, D., Mas, Y., Bennetau, B., Dejous, C., Multipurpose Love acoustic wave immunosensor for bacteria, virus or proteins detection. IRBM in-press.

Mukdadi, O.M., Desai, Y.M., Datta, S.K., Shah, A.H., Niklasson, A.J., 2002. Elastic guided waves in a layered plate with rectangular cross section. Journal of the Acoustical Society of America 112(5), 1766 - 1779.

Mukdadi, O.M., Datta, S.K., 2003. Transient ultrasonic guided waves in layered plates with rectangular cross section. Journal of Applied Physics 93 (11), 9360 - 9370.

Nakamura, K., 1992. Antipolarity domain formed by heat treatment of ferroelectric crystals on their applications. Japanese Journal of Applied Physics 31, sup. 31-1, 9-13.

Nakamura, K., Koyama, K., Odakura, S., Yamada, K., Saito, S., 2005. $\mathrm{LiNbO}_{3}$ ultrasonic transducers with and inverted-domain layer for radiation to solid media. Japanese Journal of Applied Physics 44(6B), 4482-4484.

Nakamura, K., Fukazawa, K., Yamada, K., Saito, S., 2003. Broadband ultrasonic transducers using $\mathrm{LiNbO}_{3}$ plate with a ferroelectric inversion layer. IEEE Transaction on Ultrasonics Ferroelectrics and Frequency Control 50(11), 15581562.

Nayfeh, A. H., 1995. Wave propagation in layered anisotropic media. Amsterdam: Elsevier.

Nayfeh, A.H., Chimenti, D.E., 1984. Reflection of finite acoustic beams from loaded and stiffened half-spaces. Journal of the Acoustical Society of America 75, 13601368.

Nayfeh, A.H., Chimenti, D.E., 1988. Propagation of guided waves in fluid-coupled plates of fiber reinforced composite. Journal of the Acoustical Society of America 83, 1736-1743.

Negishi K., 1987. Existence of negative group velocities in Lamb waves. Japanese Journal of Applied Physics 26 (sup 26-1), 171-173.

Onoe, M., Tiersten, H.F., 1963. Resonance frequencies of finite piezoelectric ceramic vibrators with high electromechanical coupling. IEEE Transactions on Ultrasonics Engineering 7, 32-39.

Pan, E., Datta, S.K., 1999. Ultrasonic waves in multilayered superconducting plates. Journal of Applied Physics 86, 543-551.

Pauley, K.E., Dong, S.B., 1975. Analysis of plane waves in laminated piezoelectric plates. Wave Electronics 1, 265-285.

Powell, D.J., Hayward, G., Ting, R.T., 1998. Unidimensional modeling of multi-layered piezoelectric transducer structures. IEEE Transaction on Ultrasonics Ferroelectrics and Frequency Control 45(3), 667 
Predoi, M.V., Castaings, M., Hosten, B., Bacon, C., 2007. Wave propagation along transversely periodic structures. Journal of the Acoustical Society of America 121, 1935-1944.

Raimbault, V., Rebiere, D., Dejous, C., 2008. A microfluidic surface wave sensor platform: Application to high viscosity measurements. Materials Science and Engineering C 28, 759-764.

Ramesh, R., Durga Prasad, C., Vinod Kumar, T.K., Gavane, L.A., Vishnubhatla, R.M.R., 2006. Experimental and finite element modelling studies on single-layer and multi-layer 1-3 piezocomposite transducers. Ultrasonics 44, 341-349.

Redwood, M., 1961. Transient performance of a piezoelectric transducer. Journal of the Acoustical Society of America 33, 527-36.

Reid, J.M., Wild, J.J., 1952. Ultrasonic ranging for cancer diagnosis. Electronics 25, 136138.

Ricco, A. J., Martin, S. J., 1987. Acoustic wave viscosity sensor. Applied Physics Letters 50, 1474-1476.

Saitoh, S., Izumi, M., Mine, Y., 1995. IEEE Trans. A dual frequency ultrasonic probe for medical applications. IEEE Transaction on Ultrasonics Ferroelectrics and Frequency Control 42(2), 294-300.

Siao, J.C-T., Dong, S.B., Song, J., 1994. Frequency spectra of laminated piezoelectric cylinders. ASME Journal of Vibration and Acoustics 16, 364-370.

Sivaramakrishnan, S., Rajamani, R., Smith, C.S., McGee, K.A., Mann, K.R., Yamashita, N., 2008. Carbon nanotube-coated surface acoustic wave sensor for carbon dioxide sensing. Sensors and Actuators B, in-press.

Spencer, K.T., Bernarz, J., Rafter, P.G., Korcarz, C., Lang, R.M., 1998. Use of harmonic imaging without electrocardiographic contrast to improve two-dimensional image quality. American Journal of Cardiology 82, 794-799.

Taciroglu, E., Liu, C.W., Dong, S.B., Chun, C.K., 2004. Analysis of laminated piezoelectric circular cylinders under axisymmetric mechanical and electrical loads with a semi-analytic finite element method. International Journal of Solids and Structures 41, 5185-5208.

Tiersten, H.F., 1969, Linear Piezoelectric Plates, Plenum Press, New York, USA.

Tranquart, F., Grenier, N., Eder, V., Pourcelot, L., 1999. Clinical use of ultrasound tissue harmonic imaging. Ultrasound in Medicine \& Biology 25, 889-894.

Turton, A., Bhattacharyya, D., Wood, D., 2006. Liquid density analysis of sucrose and alcoholic beverages using polyimide guided Love-mode acoustic wave. Measurement Science and Technology 17, 257-263.

von Ramm, O.T., Smith, S.W., 1978. A multiple frequency array for improved diagnostic imaging. IEEE Transactions on Sonics \& Ultrasonics 25 (6), 340-345.

Vos, H.J., Frijlink, M.E., Droog, E., Goertz, D.E., Blacquiere, G., Gisolf, A., de Jong, N., van der Steen, A.F.W., 2005. Transducer for harmonic intravascular ultrasound imaging. IEEE Transaction on Ultrasonics Ferroelectrics and Frequency Control 52(12), 2418-2422.

Wang, D.Y., Chan, H.L-W., 2003. A dual frequency ultrasonic transducer based on BNBT-6/epoxy 1-3 composite. Materials Science and Engineering B 99, 147-150. 
Wang, W., Lee, K., Woo, I., Park, I., Yang, S., 2007. Optimal design on SAW sensors for wireless pressure measurement based on reflective delay line. Sensors and Actuators A139, 2-6.

Wenzel, S. W., White, R. M., 1988. A multisensor employing an ultrasonics Lamb-wave oscillator. IEEE Transactions on Electron Devices 35, 735-743.

Wenzel, S. W., White, R. M., 1989. Analytic comparison of the sensitivities of bulkwave, surface-wave, and flexural plate-wave ultrasonic gravimetric sensors. Applied Physics Letters 54, 1976-1978.

White, R.M., Voltmer, F.W., 1965. Direct piezoelectric coupling to surface elastic waves. Applied physics letters 7, 314.

White, R.M., Wenzel, S.W., 1988. Fluid loading of Lamb wave sensor. Applied physics letters 52 (20), 1653-1655.

Wilm, M., Armati, R., Daniau, W., Ballandras, S., 2004. Cross-talk phenomena in a 1-3 connectivity piezoelectric composite. Journal of the Acoustical Society of America 116, 2948-2955.

Wu, J., Zhu, Z., 1995. An alternative approach for solving attenuated leaky Rayleigh waves. Journal of the Acoustical Society of America 37, 3191-31-93.

Wu, J., Zhu, Z., 1992. Propagation of Lamb waves in a plate bordered with layers of a liquid Journal of the Acoustical Society of America 91, 861-867.

Wu, T. T., Chen, Y. Y., Chou, T. H., 2008. A high sensitivity nanomaterial based SAW humidity sensor. Journal of Physics D: Applied Physics 41, 085101.

Yang C.H., Chimenti, D.E., 1995. Guided plate waves in piezoelectrics immersed ina dielectric fluid. I. Analysis. Journal of the Acoustical Society of America 97, 2103-2109.

Yang C.H., Shue, C.J., 2001. Guided waves propagating in a piezoelectric plate immersed in a conductive fluid. NDT\&E international 34, 199-206.

Yang C.H., Lai, Y.A., 2005. Dielectric and mechanical loading effects of a fluid on Lamb waves propagating on an immersed piezoelectric plate. Journal of Mechanics 21, 179-186.

Yang, C.H., Tsai, K.Y., 2006. A study on the dispersions of piezoelectric plate with laser ultrasound measurement and theoretical modeling. Ultrasonics 44, 807-811.

Zhang, Z., Feng, C., Liew, K.M., 2006. Three-dimensional vibration analysis of multilayered piezoelectric composite plates. International Journal of Engineering Science 44, 397-408.

Zhou Q.F., Cannata, J., Kirk-Shung, K., 2006. Design and modeling of inversion layer ultrasonic transducers using $\mathrm{LiNbO}_{3}$ single crystal. Ultrasonics 44, 607-611.

Zhou, Q., Cannata J.M., Guo H., Huang C., Marmarelis V.Z., Kirk Shung, K., 2005. Half-thickness inversion layer high-frequency ultrasonic transducers using $\mathrm{LiNbO}_{3}$ single crystal. IEEE Transaction on Ultrasonics Ferroelectrics and Frequency Control 52(1), 127-133. 